\title{
Kenya: Request for a Three-Year Arrangement Under the Extended Credit Facility- Staff Report; Press Release on the Executive Board Discussion; and Statement by the Executive Director for Kenya.
}

In the context of the Kenya-request for a three-year arrangement under the extended credit facility, the following documents have been released and are included in this package:

- $\quad$ The staff report for the Kenya-Request for a Three-Year Arrangement Under the Extended Credit Facility, prepared by a staff team of the IMF, following discussions that ended on November 18, 2010, with the officials of Kenya on economic developments and policies. Based on information available at the time of these discussions, the staff report was completed on January 14, 2011. The views expressed in the staff report are those of the staff team and do not necessarily reflect the views of the Executive Board of the IMF.

- $\quad$ A Press Release summarizing the views of the Executive Board as expressed during its January 31, 2011 discussion of the staff report that completed the request and/or review.

- A statement by the Executive Director for Kenya.

The documents listed below have been or will be separately released.

Letter of Intent sent to the IMF by the authorities of Kenya*

Memorandum of Economic and Financial Policies by the authorities of

Kenya*

Technical Memorandum of Understanding*

*Also included in Staff Report

The policy of publication of staff reports and other documents allows for the deletion of market-sensitive information.

\author{
Copies of this report are available to the public from \\ International Monetary Fund • Publication Services \\ $70019^{\text {th }}$ Street, N.W. • Washington, D.C. 20431 \\ Telephone: (202) 623-7430 • Telefax: (202) 623-7201 \\ E-mail: publications@imf.org Internet: http://www.imf.org
}

\section{International Monetary Fund Washington, D.C.}


INTERNATIONAL MONETARY FUND

KENYA

\section{Request for a Three-Year Arrangement Under the Extended Credit Facility}

Prepared by the African Department

(In consultation with other Departments)

Approved by Saul Lizondo and Dominique Desruelle

January 14, 2011

Fund arrangement: In the attached letter, the Kenyan authorities are requesting a three-year arrangement under the Extended Credit Facility (ECF).

Discussions: Preliminary discussions were held in Nairobi in September 13-27, 2010 and final discussions took place during November 3-18, 2010. The mission met with Prime Minister Odinga, Deputy Prime Minister and Minister of Finance Kenyatta, Central Bank Governor Ndung'u, the Parliament's Finance Committee, other senior officials, representatives of the private sector including financial institutions, and the donor community. The staff team comprised Messrs. Fanizza (head), Milkov, Morales, and Ms. Sanya (all AFR) and Mrs. Nkusu (SPR). Mr. Gudmundsson (Resident Representative) participated in the discussions.

Objective: To protect the external position, while allowing a needed fiscal adjustment to take place in a gradual manner over a three-year horizon.

Access: SDR 325.7 million (120 percent of quota). An initial disbursement of SDR 65.1 million will become available upon approval of this request by the Executive Board.

Term and phasing: 36 months, with first and second reviews scheduled to take place by July 15 and October 15, 2011 respectively, and semiannual reviews thereafter.

Conditionality: Focused on the program's objectives of reserves build up, improvement in the primary fiscal balance, public finance management reform, tax reform, and strengthening of the financial system.

Safeguards assessment: The new arrangement will require an update of the safeguards assessment by the time of the first review.

Poverty Reduction Strategy Paper (PRSP): The authorities issued their current PRSP in October 2009. 
Glossary





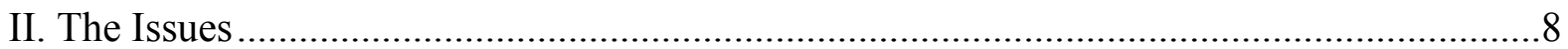

A. Build Up International Reserves ................................................................. 8

B. Need for Gradual Fiscal Consolidation ..........................................................

C. The New Constitution Will Favor Major Governance Reforms ...........................10

III. The Economic Program 2011-13 .............................................................................. 11

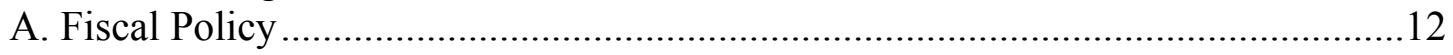

B. Monetary and Financial Policies .............................................................. 18

IV. Program Modalities and Risks .............................................................................21

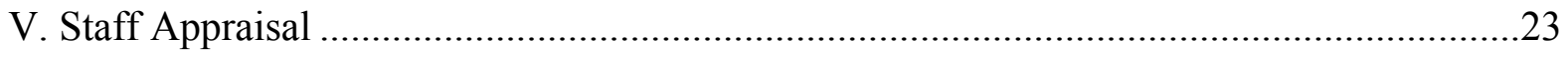

Tables

1. Selected Economic Indicators, 2008/09-2013/14 ....................................................25

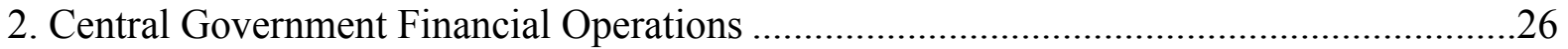

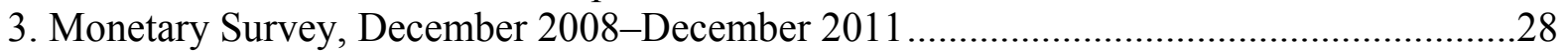

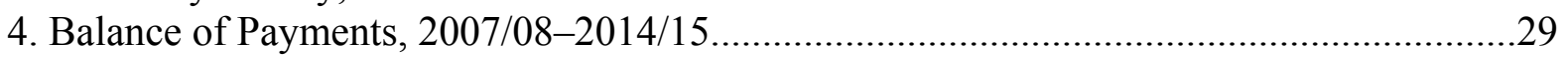

5. Financial Soundness Indicators of the Banking Sector..................................................30

6. Proposed Timing of Purchases and Reviews under the 2011-14 ECF Arrangement..........31

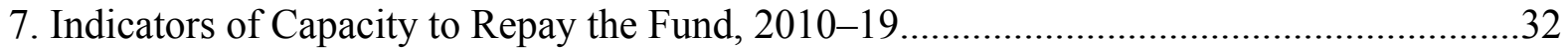

Figures

1. Recent Developments and Outlook .............................................................................13

2. Selected Fiscal Indicators in Kenya and Other Sub-Saharan African Countries.................15

3. Credit Market Developments in Kenya and Comparable Countries, 2007-10 .................19

Boxes

1. Assessing the Adequacy of Kenya's Gross International Reserves....................................8

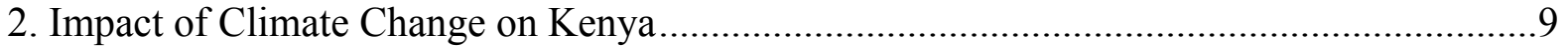



4. The 2008 Ex-Post Assessment of Longer-Term Program Engagement ............................11

5. Medium-Term Debt Management Strategy ............................................................. 17

6. Financial Sector Deepening and Modernization..........................................................20

Appendixes

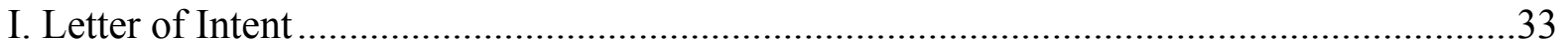

Attachment 1. Memorandum of Economic and Financial Policies for 2011-13 .........35

Attachment 2. Technical Memorandum of Understanding.....................................46 


\section{GLOSSARY}

\begin{tabular}{|c|c|}
\hline AfDB & African Development Bank \\
\hline AFRITAC & African Technical Assistance Center \\
\hline AIA & Appropriations in Aid \\
\hline BPM5 & Fifth Edition of the Balance of Payments Manual \\
\hline CBK & Central Bank of Kenya \\
\hline CIRRs & Commercial interest reference rates \\
\hline CPI & Consumer price index \\
\hline CPIA & Country Policy and Institutional Assessment \\
\hline DFID & Department for International Development \\
\hline DPL & Development policy loan \\
\hline DSA & Debt Sustainability Analysis \\
\hline EAC & East African Community \\
\hline $\mathrm{ECF}$ & Extended Credit Facility \\
\hline EDDI & Enhanced Data Dissemination Initiative \\
\hline EMC & Emerging market countries \\
\hline ESF & Exogenous Shocks Facility \\
\hline FAD & Fiscal Affairs Department \\
\hline FIS & Foreign Investment Survey \\
\hline FLSTAP & Financial and Legal Sector Technical Assistance Loan \\
\hline FSAP & Financial Sector Assistance Program \\
\hline GDDS & General Data Dissemination System \\
\hline GFR & Gross financing requirements \\
\hline GFS & Government Finance Statistics \\
\hline GFSM & Government Finance Statistics Manual \\
\hline GIR & Gross international reserves \\
\hline HIPC & Heavily indebted poor countries \\
\hline IFMIS & Integrated Financial Management System \\
\hline IFS & International Financial Statistics \\
\hline IIP & International investment position \\
\hline KNBS & Kenya National Bureau of Statistics \\
\hline KRA & Kenya Revenue Authority \\
\hline Ksh & Kenyan Shilling \\
\hline LATF & Local Authorities Trust Fund \\
\hline LICs & Low income countries \\
\hline MCM & Monetary and Capital Markets Department \\
\hline MDGs & Millennium development goals \\
\hline MEFMI & $\begin{array}{l}\text { Macroeconomic and Financial Management Institute of } \\
\text { Eastern and Southern Africa }\end{array}$ \\
\hline MEFP & Monetary and Economic Financial Program \\
\hline MFI & Microfinance institution \\
\hline
\end{tabular}




$\begin{array}{ll}\text { MSME } & \text { Micro, small, and medium enterprise } \\ \text { MTDS } & \text { Medium-Term Debt Management Strategy } \\ \text { MTP } & \text { Medium-Term Plan } \\ \text { NDA } & \text { Net domestic assets } \\ \text { NIR } & \text { Net international reserves } \\ \text { NPV } & \text { Net present value } \\ \text { NSIS } & \text { National Security Intelligence Service } \\ \text { NSSF } & \text { National Social Security Fund } \\ \text { ODC } & \text { Other depository corporation } \\ \text { OECD } & \text { Organization for Economic Cooperation and Development } \\ \text { PFM } & \text { Public financial management } \\ \text { PPG } & \text { Public and publicly guaranteed } \\ \text { PPP } & \text { Public Private Partnership } \\ \text { PRGF } & \text { Poverty Reduction Growth Facility } \\ \text { PRSP } & \text { Poverty Reduction Strategy Paper } \\ \text { RAC } & \text { Rapid Access Component } \\ \text { RBA } & \text { Retirement Benefit Authority } \\ \text { ROSC } & \text { Report on Observance of Standards and Codes } \\ \text { SACCOs } & \text { Savings and Credit Cooperatives } \\ \text { SDR } & \text { Special Drawing Rights } \\ \text { SRF } & \text { Standardized report forms } \\ \text { SSA } & \text { Sub-Saharan Africa } \\ \text { STA } & \text { Statistics Department } \\ \text { TMU } & \text { Technical memorandum of understanding } \\ \text { TRC } & \text { Tax Reform Commission } \\ \text { VAT } & \text { Value-added tax } \\ \text { WB } & \text { World Bank } \\ & \end{array}$




\section{EXECUTIVE SUMMARY}

Kenya's economy is bouncing back following multiple shocks between 2008 and 2009. Fiscal stimulus has boosted the recovery, especially in construction, and benign weather conditions have supported a rebound in agriculture. The ratification of the new constitution in August 2010 has spurred confidence, and private investment is expected to accelerate and support annual growth rates close to 7 percent over the medium term. The mobile-banking revolution has brought large segments of the population to gain access to banking services and has injected dynamism in financial intermediation.

At the same time, sustaining high growth will require addressing macroeconomic vulnerabilities. The ongoing recovery will weigh on Kenya's weak external position, and the rise in debt burden resulting from the fiscal stimulus needs to be reversed. However, fiscal consolidation needs to take account of the need to implement the new constitution and to invest in infrastructure, including geothermal power generation.

The new constitution provides a unique opportunity to address deep-seated social and institutional problems, and bolster social cohesion. Widespread support for the new constitution creates the conditions for meaningful reforms, including on fiscal decentralization, the public expenditure framework, and land ownership. A successful implementation of constitutional provisions will also address governance by strengthening the judiciary and laying the ground for an overhaul of the public management system that would effectively reduce the scope for corruption.

The authorities are requesting a three-year ECF arrangement. Their program aims at boosting international reserves while adopting a gradual fiscal adjustment over a three-year horizon. The arrangement would provide a reserve cushion to reduce vulnerabilities to external shocks, including a projected deterioration of the terms of trade in the next two years. The program will also ensure fiscal discipline during the implementation of fiscal decentralization, while keeping inflation low and maintaining the floating exchange rate regime.

The program targets a gradual reduction in the central government primary balance through tax reform and strict control of current spending to bring the debt-to-GDP ratio below 45 percent at the end of the program period. Tax measures include the reform of the value-added tax (VAT) and the income tax to broaden their bases and improve compliance. The new framework for expenditure management and control will allow the government to reign in current spending while providing scope for allocating resources for the implementation of the constitution over the next two years. The government will also continue pursuing the poverty reduction objectives in line with the PRSP. 


\section{BACKGROUND}

1. Kenya's economy is bouncing back, after faltering in 2008-09. Two consecutive droughts, social strife, and the global economic downturn halted the strong growth record that Kenya had enjoyed since 2004. The 2007 post-election violence manifested social tensions poorly attended by a centralized political system. The global downturn hurt tourism and nontraditional exports. Good rains and a strong tourist season have lifted growth; and the ratification of the new constitution has boosted confidence:

- We project real GDP growth at 5 percent in 2010, up from 2.6 percent in 2009.

Benign weather conditions have favored a rebound in agriculture growth; and the government's renewed efforts to upgrade infrastructure have boosted construction activity.

- International reserves have remained at 3.3 months of imports. Unlike other countries in the region, political uncertainty has kept capital inflows subdued. Foreign reserves coverage has remained flat despite Fund support under the ESF, the 2009 Special Drawing Rights (SDR) allocation, and an improved current account resulting from higher tea exports, tourism receipts, and remittances.

- Public debt now lies above the 45 percent debt-to-GDP ceiling (40 percent in net present value) set by Kenya's mediumterm debt-management strategy.

Following an expansionary budget in 2009/10, large investment in infrastructure, one-off security-related outlays, and the initial cost of implementing the constitution are projected to keep the central government primary deficit at
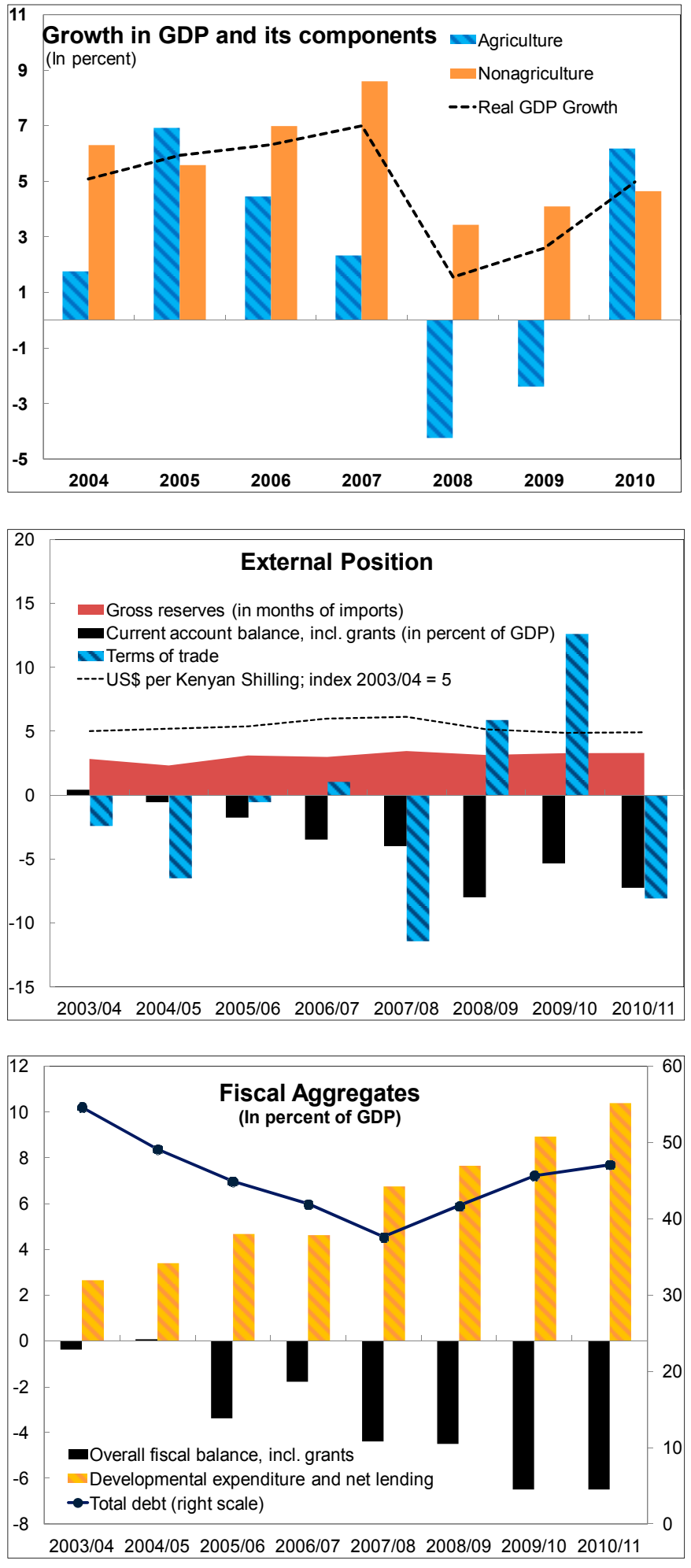
4 percent of GDP for a second consecutive year in 2010/11. 
- Inflation has remained below the 5 percent central bank target, despite abundant liquidity and low interest rates. Inflation has generally declined through 2010, reaching 4.5 percent in December; and low short-term interbank, repo, and T-bill rates supported a pickup in credit growth to 23 percent by September 2010 .

- The downward shift in the government bond yield curve suggests that inflationary expectations have improved. In particular, the remarkable drop in interest rates at the long end of the curve, at a time of continuous increasing bond supply, illustrates both the expectation of stability and the depth of the bond market.

- Increased monetization and expanded access to banking services explain a pickup in credit growth.

However, credit growth has remained below deposit growth throughout 2010 reflecting banks' cautious lending policies and strong preference for high liquidity. The high share of low-risk assets has translated into robust soundness indicators, while innovations in retail banking have favored increased access to financial services to previously unbanked population.



\section{THE ISSUES}

2. Kenya's international reserves have remained low. The ongoing domestic-led recovery and an expected deterioration of the terms of trade are likely to further weigh on the external position. The pace of fiscal adjustment should take account of the need to implement the new constitution and to invest in infrastructure (including geothermal power generation). The new constitution should cement social cohesion by decentralizing fiscal responsibilities to the regions and create the conditions necessary to address the governance issues that have held back the country's growth potential.

\section{A. Build Up International Reserves}

3. Kenya requires building a larger international reserve buffer. The external current account deficit is expected to widen to about 7 $1 / 2$ percent of GDP in the next two years, because of increased demand for imports of goods and services arising from higher growth and a cumulative 15 percent deterioration of Kenya's terms of trade. The current international reserve buffer appears insufficient to face additional external pressures (Box 1).

\section{Box 1. Assessing the Adequacy of Kenya's Gross International Reserves}

Kenya's reserves remain relatively low despite the authorities' reserves buildup efforts. The impact of the global financial crisis and two successive droughts brought down reserves in 2008/2009. The Fund's 2009

Exogenous Shocks Facility (ESF) disbursement and SDR allocations helped boost reserves to $31 / 2$ months of imports by end-2009, from almost three months a year earlier.

Several factors suggest that a further accumulation of reserves would be desirable:

- $\quad$ Reserves are low relative to alternative cross-country comparator groups. Kenya's reserves are at the low end relative to East African Countries (EAC), Sub-Saharan Africa (SSA), all nonfuel exporters, or selected Emerging Market Countries (EMC).

- Model-based estimates suggest that Kenya's reserves fall short of predicted levels.

- Kenya is more vulnerable to volatile private capital flows than many Low Income Countries (LICs) holding larger reserves buffers, reflecting its relatively deep and more open financial markets.



Note: 15 EMC plus Kenya refers to the median of a sample including Kenya and 15 emerging market economies with a level of broad money-to-GDP close to that of Kenya. GFR stands for gross financing requirements computed as following year's current account deficit plus short-term debt at residual maturity. Gross International Reserves (GIR)-to-GFR and GIR-to-broad money are scaled by 10 . They are not available for Sub-Saharan Africa and nonfuel exporters.

\begin{tabular}{|lc}
\hline \multicolumn{2}{|c}{$\begin{array}{c}\text { Explaining Demand for Reserves in LIC } \\
\text { and Selected EMC }\end{array}$} \\
\hline \multicolumn{2}{c}{$\begin{array}{c}\text { Dependent Var. } \\
\text { Reserves-to-GDP }\end{array}$} \\
\hline Explanatory Variables & 0.4837 \\
\hline Res-to-GDP & 5.4512 \\
Log population & -3.7889 \\
Log(real per cap. GDP) & 0.0138 \\
Trade-to-GDP & 0.1591 \\
Broad money-to-GDP & 5.5954 \\
Exchange rate volatility & 0.0196 \\
Private inflows-to-GDP & 0.0004 \\
Short-term external debt-to-GDP & 11.8456 \\
Constant & 1,661 \\
Number of observations & \\
\hline
\end{tabular}

Estimation based on sample of $78 \mathrm{LIC}$ and EMC (excl. China and India) during 1985-2009. Arellano-Bond dynamic panel approach used. All variables are significant at standard levels.


(In billions U.S. dollars)
Kenya: Gross Reserves--Predicted vs. Actual 


\section{B. Need for Gradual Fiscal Consolidation}

\section{Fiscal policy should curb the recent growth in debt while attending investment needs and commitments associated with the implementation of the constitution:}

- $\quad$ The recent increase in the debt-to-GDP ratio threatens fiscal sustainability and should be gradually reversed.

- $\quad$ The room for tightening fiscal policy is limited, as the government steps up infrastructure investment to remove important growth hurdles.

- $\quad$ Expanded geothermal power capacity is required to sustain growth, by reducing Kenya's dependence on oil imports and hydropower generation, enhancing its ability to deal with climate-change shocks (Box 2).

- $\quad$ The implementation of the constitution entails new fiscal challenges, from initial implementation spending to managing regional demands once fiscal decentralization is rolled out.

\section{Box 2. Impact of Climate Change on Kenya}

Some studies show that climate change has already afflicted damage on Kenya. ${ }^{1}$ In the last 50 years, the country has experienced a general warming across regions. Periodic droughts and floods have become more frequent and intense and have caused major economic disruptions.

Droughts have hit hydro electrical power generation with disruptive effects on industrial production and tourism. They have also damaged a large part of agricultural production and livestock and reduced water availability for household and industrial use. Floods have in turn hurt infrastructure, health, agriculture and tourism in the coastal areas.

Kenya: Increase in Average Temperatures Since 1960
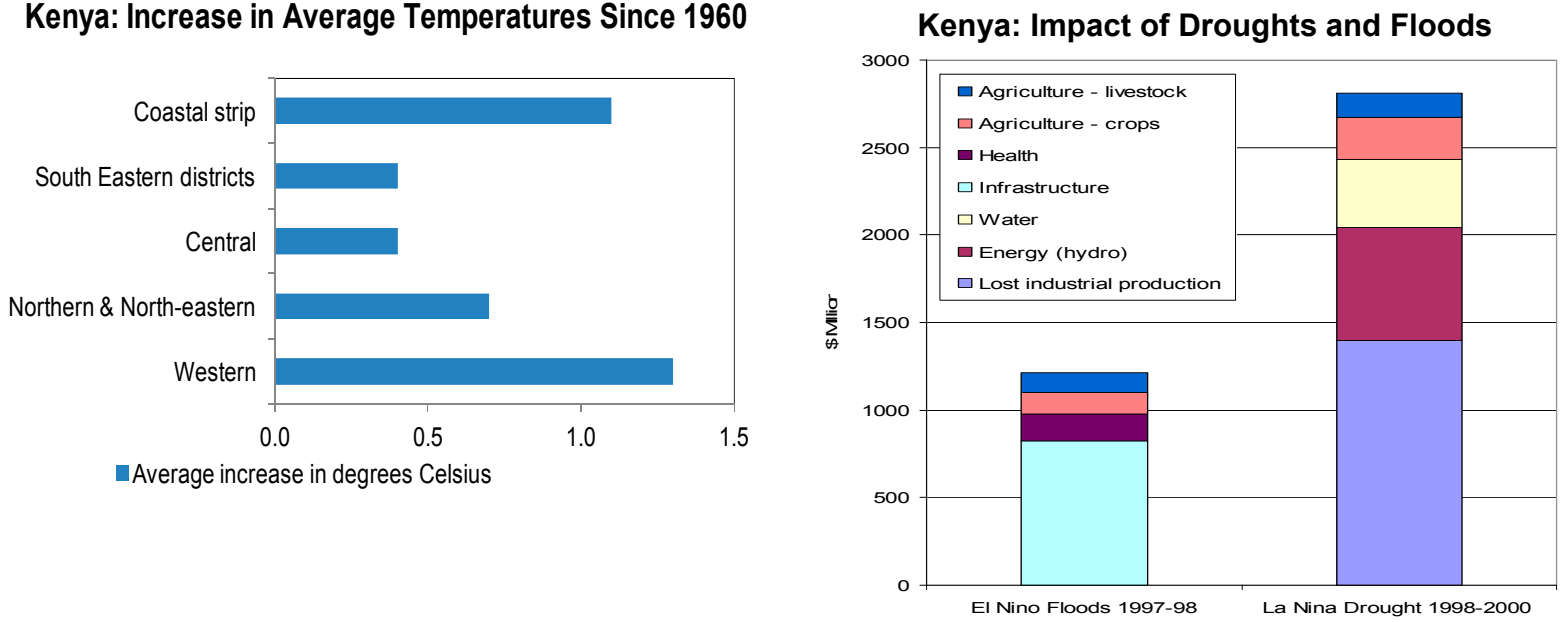

- The El Niño flood in 1997-98 had an estimated cost of \$1.2 billion. It flooded large parts of Mombasa and was the most severe flood Kenya had experienced since 1960.

- The La Niña drought in 1998-2000 was particularly lengthy and affected the Western region. The estimated total cost of the damages is $\$ 2.8$ billion.

- The drought in $2008 / 09$ prompted the authorities to import maize and fuel worth US\$1 billion to cover the shortfall in domestic food and electricity production.

Adaptation costs to climate change have been estimated at around \$500 million per year starting 2011 and increasing to \$1 billion by 2030, including investing in geothermal power generation.

1 "The Economics of Climate Change in Kenya," T. Downing, October 2009, funded by U.K. Department for International Development (DFID) and Danish International Development Agency. 


\section{The New Constitution Will Favor Major Governance Reforms}

\section{The new constitution provides an opportunity to address deep-seated social and} institutional problems (Box 3). Implementing the constitution will require introducing key legislation on fiscal decentralization, improvements in the public expenditure framework, and land ownership reform that would ensure a better and more inclusive allocation of resources across regions. The success of the constitutional referendum has raised expectations of a fast devolution of resources to the regions. ${ }^{1}$

\section{Box 3. Kenya: Constitutional Reforms}

Kenya's new constitution represents a crucial step to promote political stability and national unity, and introduces a more comprehensive system of checks and balances. The constitution will come into full force following the 2012 general and presidential elections. Main provisions include:

- the election of the President by a majority of all votes cast and a new requirement to obtain at least a quarter of votes in more than half of the 47 counties;

- $\quad$ the creation of a Senate as a new legislative chamber, a new Supreme Court, and a Judicial Service Commission;

- $\quad$ parliament involvement at earlier stages of the preparation of the budget;

- $\quad$ a new treasury law enhancing accountability and transparency in expenditure control and management;

- devolution to county level of no less than 15 percent of government revenues over a period of three years after the constitution enters into force; and

- $\quad$ harmonization of the salary scales for public servants.

\section{The new constitution provides a basis to bolster anticorruption efforts by:}

- $\quad$ establishing an independent Integrity and Anti-Corruption Committee;

- $\quad$ enhancing the independence of the judiciary, and requiring all existing justices to be vetted for suitability; and

- $\quad$ providing an opportunity for an overhaul of the public finance management system that could go a long way towards reducing the scope for corruption.

\footnotetext{
${ }^{1}$ Initial constitution implementation costs amount to around US\$40 million. Main cost components (representing about 70 percent of implementation costs) are associated with the operation of transitory commissions, review of legislation, and preparation of judicial, land, and devolution reforms.
} 


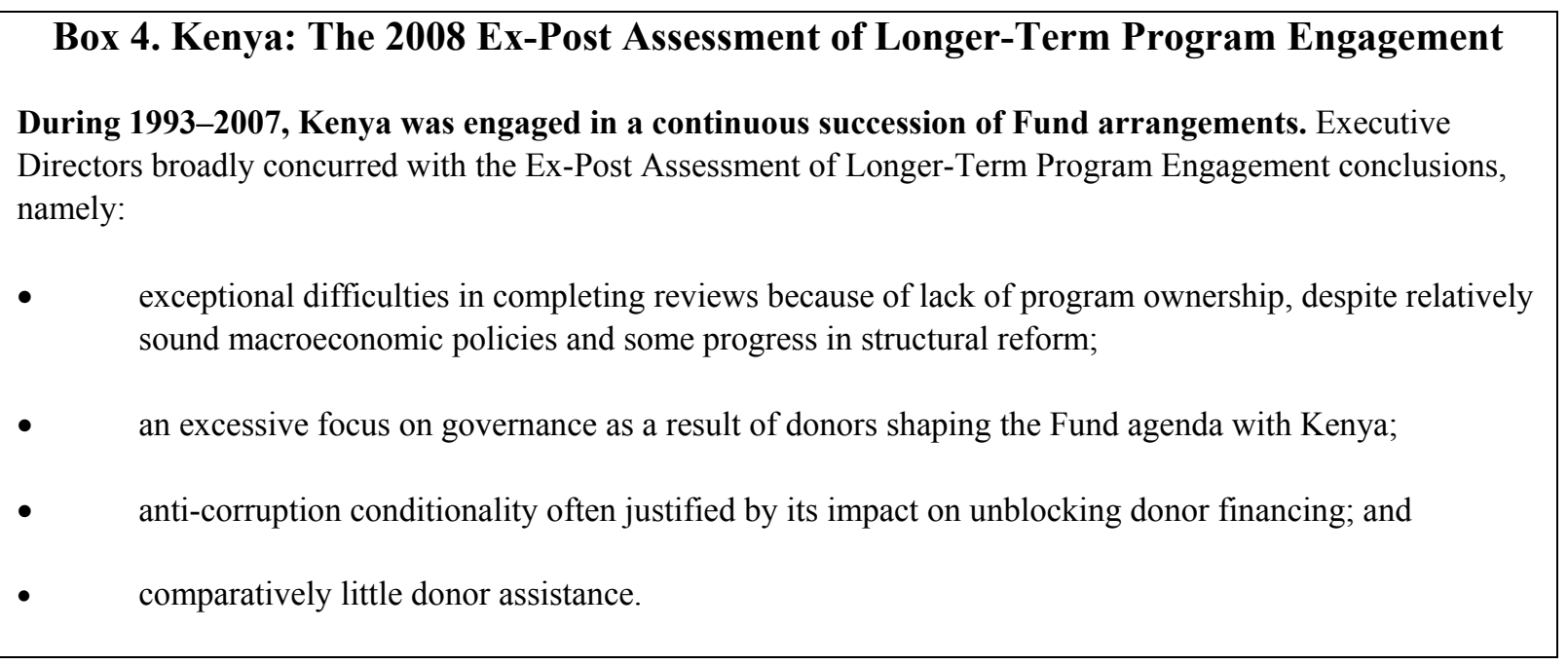

\section{The Economic Program 2011-13}

\section{A Fund arrangement in support of the authorities' program would boost} international reserves, while allowing the needed fiscal adjustment to take place in a gradual manner over a three-year horizon. The 2011-13 program strikes a balance between fiscal discipline and much-needed infrastructure spending. The international reserve buffer is projected to increase well above 4 months of prospective imports of goods and services. Supporting policies will aim at preserving stability, and creating the conditions for sustainable growth. The program envisages:

- $\quad$ protecting the external position in the face of higher domestic demand and an expected terms-of-trade shock; ${ }^{2}$

- $\quad$ implementing a gradual fiscal adjustment that protects priority spending;

- $\quad$ maintaining fiscal discipline in the implementation of the decentralization process envisaged in the constitution; and

- $\quad$ targeting low inflation and maintains the floating exchange rate regime.

\footnotetext{
${ }^{2}$ The expected deterioration in the terms of trade is due to a 14 percent annual average increase in oil prices over 2010-12, combined with a decline in Kenya's exports prices such as tea and coffee. Coffee and tea prices are projected to fall in 2011-12 by an annual average of 11 percent and 13 percent respectively.
} 
Kenya: Selected Economic Indicators, 2008/09-2013/2014 1/

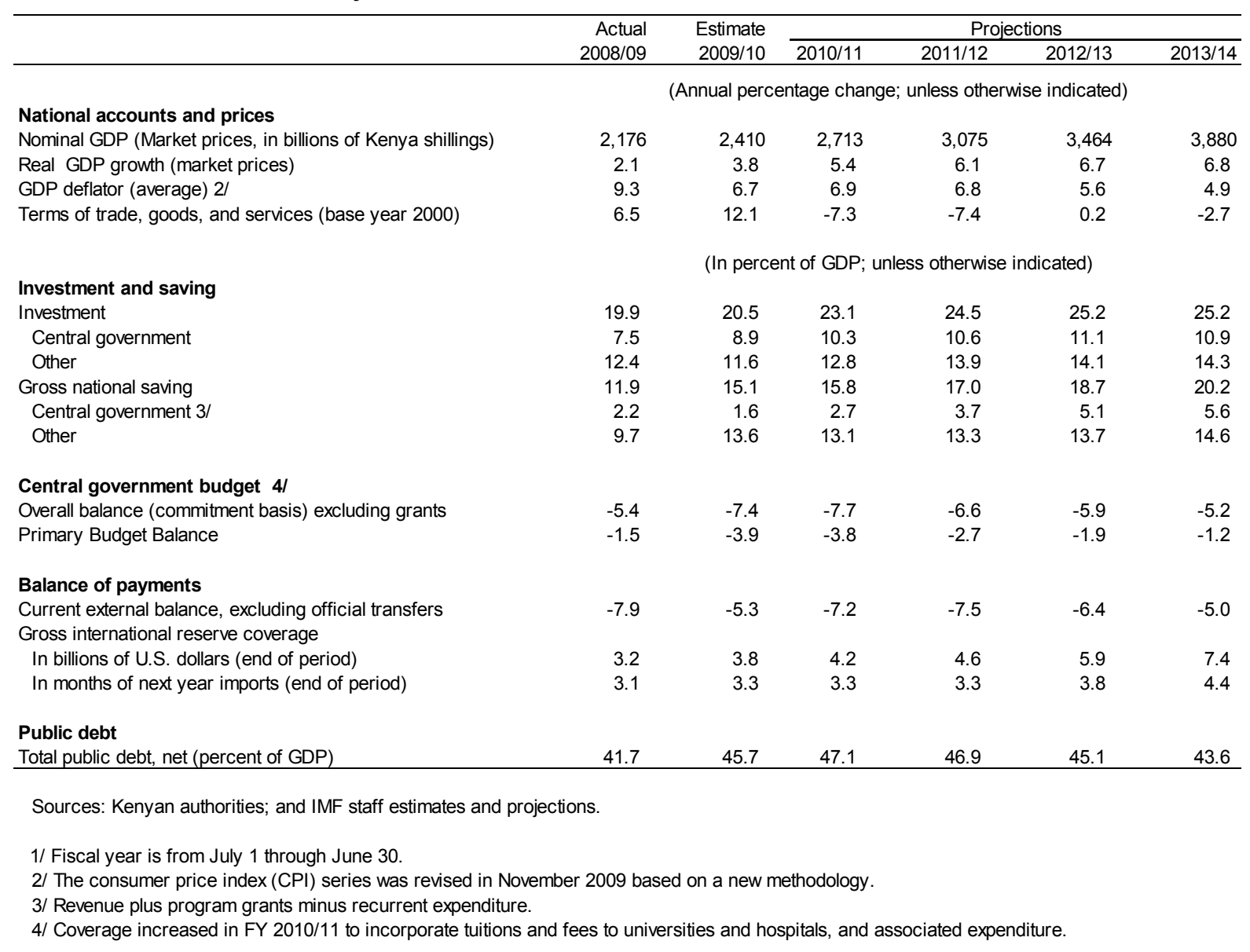

\section{The program will help sustain higher growth rates, which we cautiously project} below the 2004-07 levels (Figure 1). Over the medium term, the investment-to-GDP ratio is expected to rise to 25 percent, with public investment concentrating in energy transformation, participation in EAC regional projects, and the selective elimination of infrastructure bottlenecks. Higher private investment will support higher growth rates over the medium term.

\section{A. Fiscal Policy}

9. Policy objective: The program is based on gradual fiscal consolidation. The program targets a reduction in the central government primary deficit to 1.2 percent of GDP in 2013/14 (3.8 percent in 2010/11) through tax reform and strict control of current spending, aiming at lowering the debt burden to 45 percent of GDP in net terms by the end of the program period (Figure 2). This reduction will lower vulnerabilities by making the fiscal policy stance sustainable (DSA). The program will focus initially on the central government but will expand to encompass the general government before fiscal decentralization takes place. 
Figure 1. Kenya: Recent Developments and Outlook

Kenya's per-capita GDP dropped sharply in the last two years...

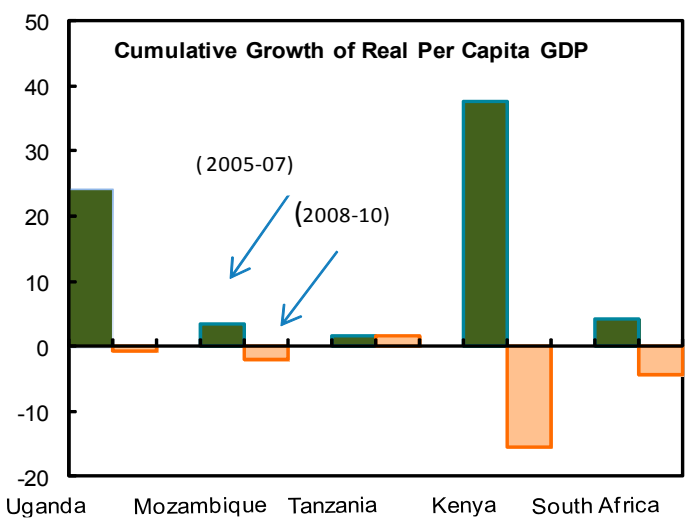

The country's market perception is favorable,...

Standard and Poor's Sovereign Foreign Rating (November 2010)

South Africa (BBB+)

Sri Lanka( $\mathrm{B}+$ )

Ukraine $(\mathrm{B}+)$

Belarus $(\mathrm{B}+)$

Uganda $(\mathrm{B}+)$

Nigeria $(\mathrm{B}+)$

Kenya(B+)

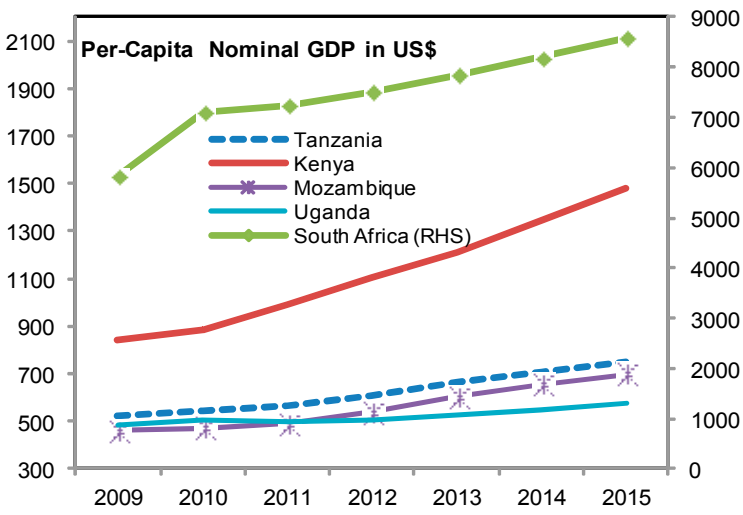

...governance indicators are improving, although corruption remains high, $1 / \ldots$.

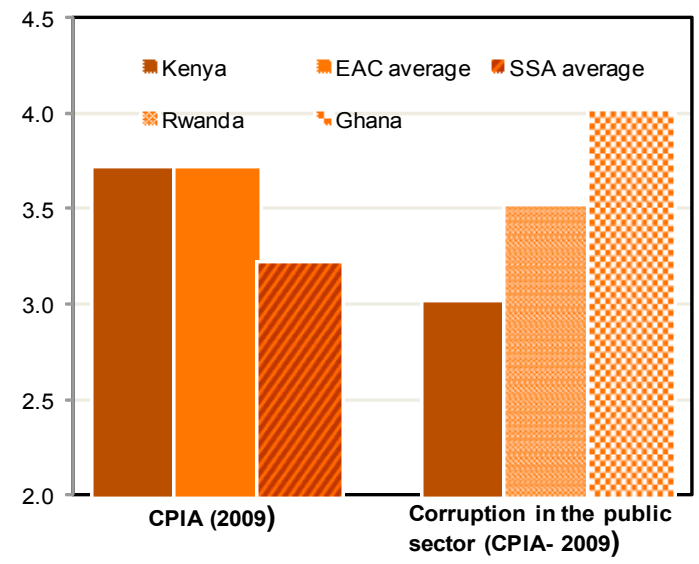

... and the business environment needs to improve.

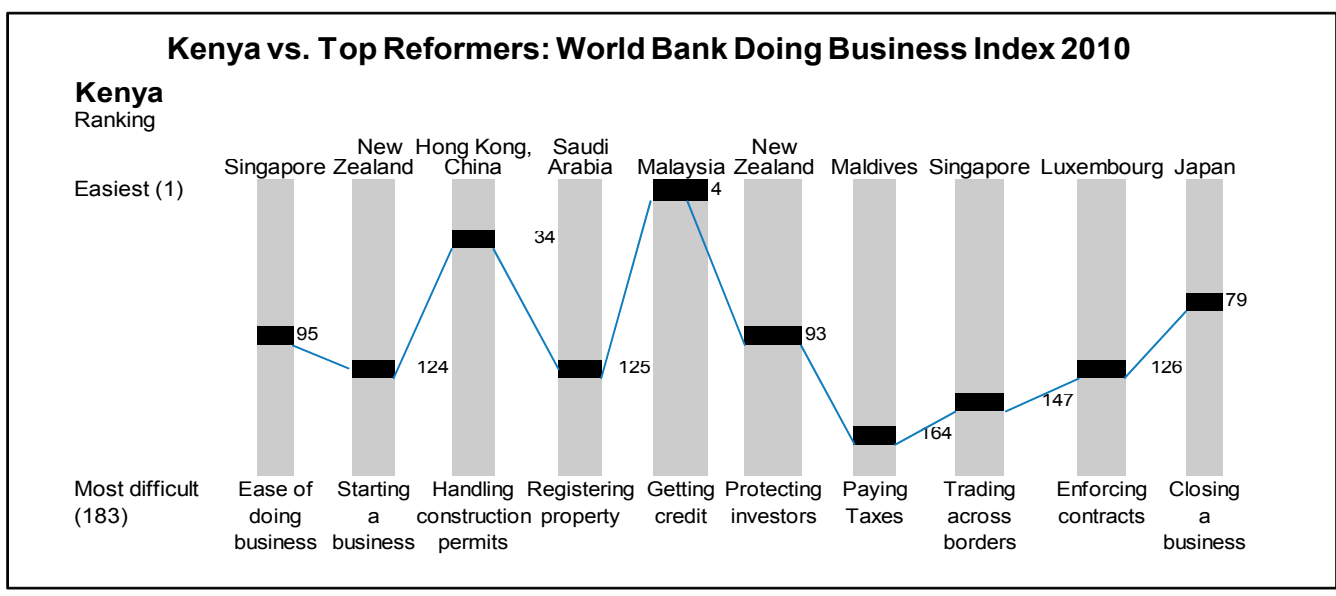

Sources: Countries' data; Country Policy and Institutional Assessment (CPIA); Standard and Poor's; World Bank; and staff estimates.

1/ A higher value of the index denotes better governance and less corruption respectively. 
Kenya: Medium-Term Program Fiscal Indicators

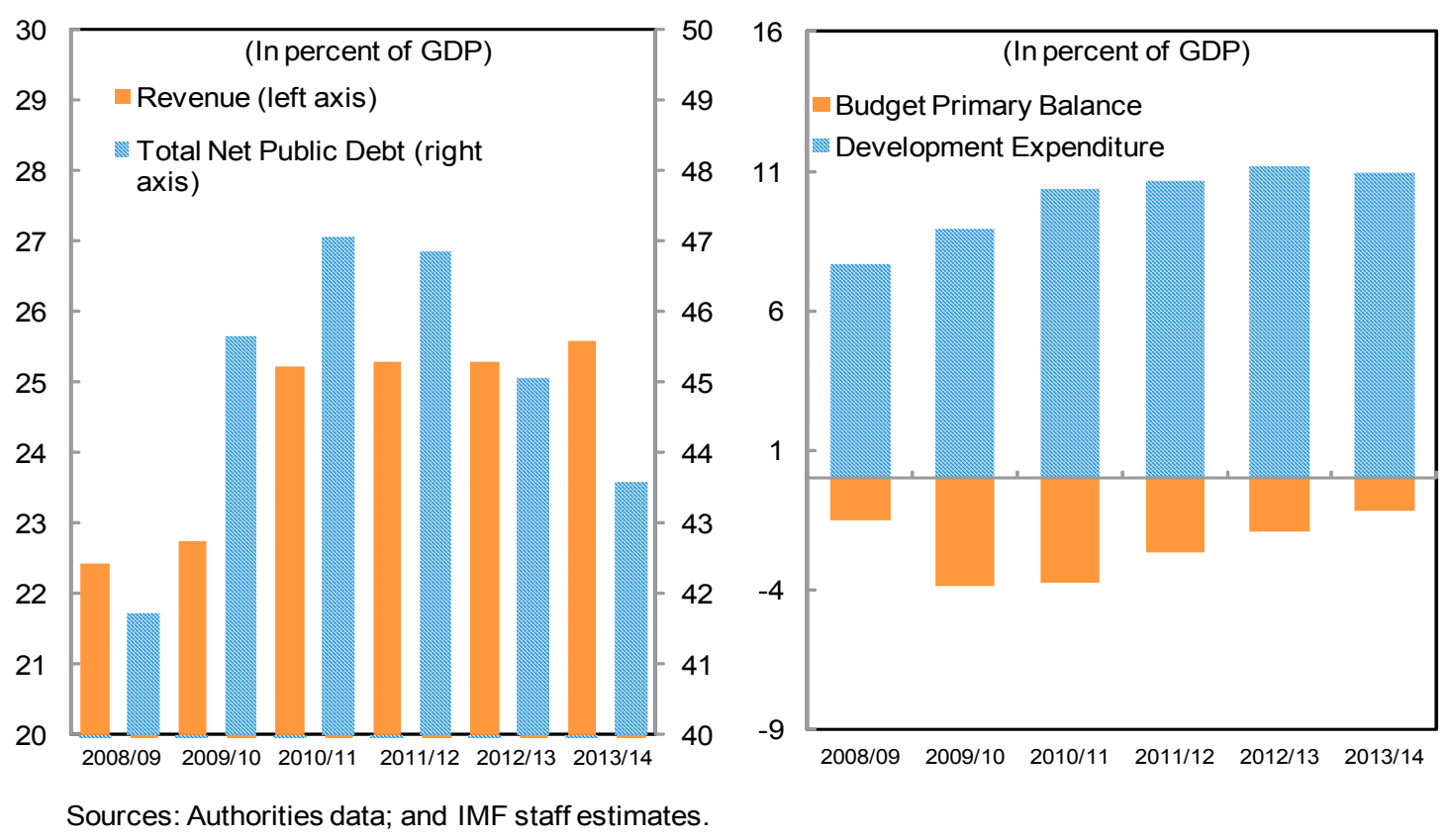

\section{Revenue measures}

10. Tax policy reform will focus on the VAT, streamlining tax incentives, and eliminating loopholes. The government will introduce a modern value-added tax (VAT) law that will improve administration and compliance (MEFP, p. 11). It will also appoint a Tax Reform Commission (TRC) that will oversee the simplification of the tax code to help improve tax compliance and raise revenue (MEFP, p. 10). Moreover, it will continue with the modernization of tax administration to help combat tax evasion (MEFP, p. 12), and upgrade custom administration in line with commitments under the EAC arrangements.

\section{Revenue projections show only a moderate increase after 2010/11 because of} one-off factors that have boosted tax collections this fiscal year. ${ }^{3}$ Tax measures are likely to have an impact of one percentage point of GDP on revenues over the program period. The reform of the VAT will be introduced in the 2012/13 fiscal year and will yield 0.4 percent of GDP. At the same time, the reform of the income tax will yield a permanent tax revenue increase of 0.6 percent of GDP.

\footnotetext{
${ }^{3}$ One-off revenue items include mainly regularization of arrears by large Kenyan corporations.
} 
Figure 2. Selected Fiscal Indicators in Kenya and Other Sub-Saharan African Countries

(2007-09 average, unless otherwise indicated)



Kenya is not an aid dependent country...

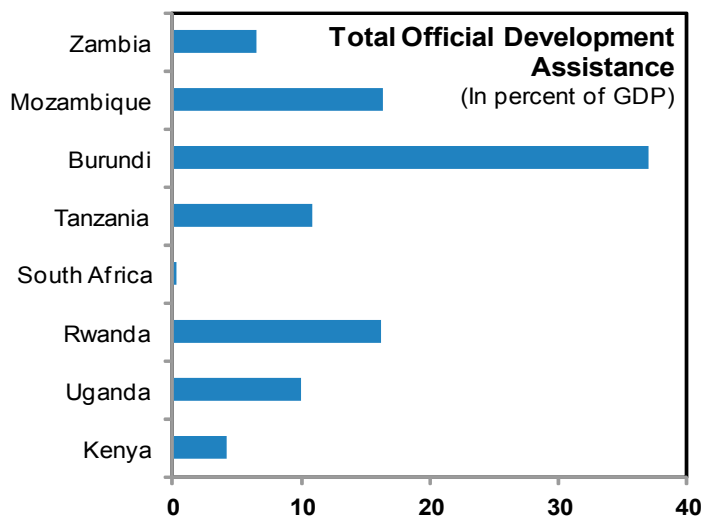

Unlike most of SSA, Kenya has not benefited from highly indebted poor countries (HIPC) ...



Sources: Authorities data; and IMF staff estimates.

1/ EAC coomprised of Burundi, Kenya, Rwanda, Tanzania, Uganda.
Yet, public investment seems relatively low.

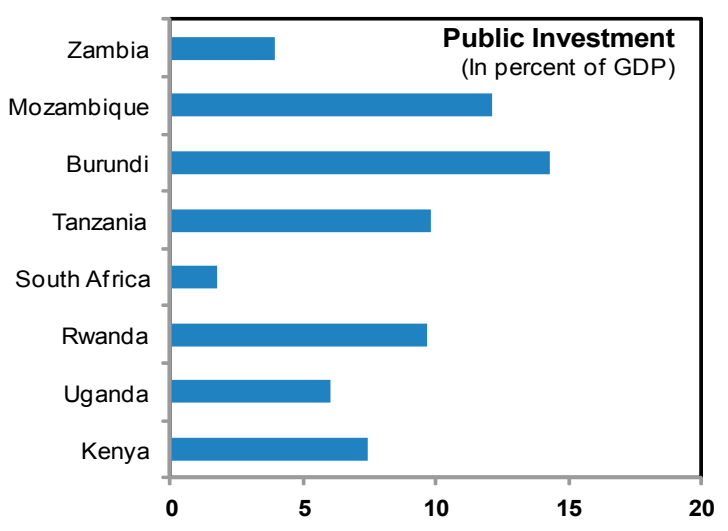

and domestic financing has partly filled the gap.



but total debt remains manageable.






\section{Expenditure measures and control}

\section{The program allows for the allocation of sufficient resources to implement the}

new constitution. In addition to the initial costs, the government has set aside an equivalent to 0.5 percent of GDP over the next two fiscal years for implementing the constitution, but a final estimate of its cost will be complete only once the legislative commissions in charge of organizing the transition to new constitutional arrangements become fully operational. ${ }^{4}$ Fiscal projections will be updated at the time of the first review to reflect corresponding revisions (MEFP, p. 9). Expenditure measures include: ${ }^{5}$

- $\quad$ no increase in real wages for public servants;

- $\quad$ rationalizing the pay grading system to eliminate existing distortions; and

- $\quad$ ensuring that fiscal decentralization (including borrowing and fiscal reporting) is consistent with the medium term fiscal policy strategy.

13. The government intends to reprioritize development expenditure to allow for (a) a prompt and effective transformation of the energy infrastructure that will help address climate-change challenges; (b) narrow the infrastructure gap; and (c) protect the vulnerable. On the latter, the government will continue pursuing the poverty reduction objectives and millennium development goals (MDGs), in line with the Poverty Reduction Strategy Paper (PRSP) (MEFP, p. 15)

\section{New public finance management (PFM) legislation will reinforce expenditure} control. The legislation will help revitalize PFM reforms under a coordinated strategy (MEFP, p. 14), and will provide the legal support for establishing a fully functioning single treasury account system for the central government to be replicated in the devolved governments when these are established. The government will fully implement an integrated financial management information system (IFMIS) comprising budget execution, cash management and procurement that will ensure effectiveness of expenditure control.

\section{Financing policy and contingent liability management}

\section{The program envisages a gradual reduction of the government's reliance on} domestic financing by increasing the use of concessional foreign financing. External financing will come mainly from concessional sources, including about US\$1.6 billion in

\footnotetext{
${ }^{4}$ These costs are partly offset by the elimination of one-off spending associated with the constitutional referendum.

${ }^{5}$ Following a one-off increase in civil service wages in 2011, wage growth will stay in line with inflation. Operational expenditure by hospitals and universities, included under "other," will be reduced as a percent of GDP by implementing strict management and control measures.
} 
project loans in the next two fiscal years, mainly from the World Bank (WB) and the African Development Bank (AfDB) (MEFP, p. 16). The government plans to issue a sovereign bond in 2012/13, but will monitor market conditions to assess the possibility of an earlier issuance, consistent with its medium-term debt-management strategy (Box 5).

\section{Box 5. Kenya: Medium-Term Debt Management Strategy}

At the time of the 2009/10 budget, Kenya adopted a Medium-Term Debt Management Strategy (MTDS) in line with the Guidance Note discussed by the Boards of the Fund and the World Bank in March 2009, helped by technical assistance (TA) from the Fund and the WB.

Main achievements are the following:

- The average maturity of domestic debt has increased to 5.5 years from 3.8 years in $\mathbf{2 0 0 8 / 0 9}$, helped by the introduction of infrastructure bonds with higher-than-average maturity.

- The newly introduced 364-day Treasury bill has been actively traded and widely demanded, providing the government with a stable source of retail funding.

- More extended use of bond re-opening has helped to establish government bonds as true benchmarks and encouraging greater liquidity in domesticcurrency securities.

- The increasing demand for government securities has allowed the government to place a large supply at declining interest rates, in nominal and real terms.

The MTDS has also helped the development of the securities market:

- Increased confidence has encouraged a more extended use of interbank repos, improving security of transactions.

- Turnover in the secondary market has increased by 230 percent in January-August 2010 relative to the same period in 2009 .

16. The government is committed to address regulatory gaps that could impede a successful implementation of the Private Public Partnership (PPP) agenda. The government will scrutinize PPP projects carefully to preserve fiscal discipline, and introduce a mechanism to properly assess fiscal risks arising from associated contingent liabilities. All guarantees arising from PPP contracts, together with all other contingent liabilities, will be integrated into the debt management process.

\section{B. Monetary and Financial Policies}

\section{Policy objectives: The program targets a gradual strengthening of the} international reserves buffer. The monetary program will favor an increase in the reserve coverage to over 4 month of prospective imports of goods and services by the end of the program period (MEFP, p. 18). The Central Bank of Kenya (CBK) will maintain a prudent policy stance, focusing on its medium term inflation target of 5 percent. It will refrain from financing the government beyond short-term use of its statutory overdraft facility. The monetary program assumes that the recent reduction in velocity and the rise in the money multiplier will continue, although at a slower pace, along with expanding financial intermediation and modernization. Private sector credit growth will remain higher than nominal GDP growth consistent with monetization trends (Figure 3).

\section{Monetary policy}

18. The CBK will closely monitor the growth in monetary aggregates to avoid renewed inflationary pressures. Main features of the monetary policy stance are as follows:

- $\quad$ Maintain the floating exchange rate regime while continuing to purchase foreign exchange as needed to achieve the foreign reserve target.

- $\quad$ Enhance coordination of monetary and fiscal policy to minimize the liquidity impact of short-term fluctuations in the government financial position with the CBK.

- $\quad$ Tighten monetary policy promptly if signs of inflationary expectations emerge.

19. The CBK will take steps to move to a forward-looking monetary framework with a view to anchoring inflation expectations:

- $\quad$ The CBK will report to the Ministry of Finance on deviations of 2 percent or more from the stated inflation target (MEFP, p. 19).

- $\quad$ The CBK Act will be revised to enhance its operational mandate within an improved framework of transparency, accountability, and operational autonomy.

- $\quad$ The CBK will upgrade its inflation forecasting capacity. 
Figure 3. Credit Market Developments in Kenya and Comparable Countries, 2007-10

Monetization in Kenya is similar to countries with comparable income level and financial stability.



Credit access is higher than in neighbor countries.

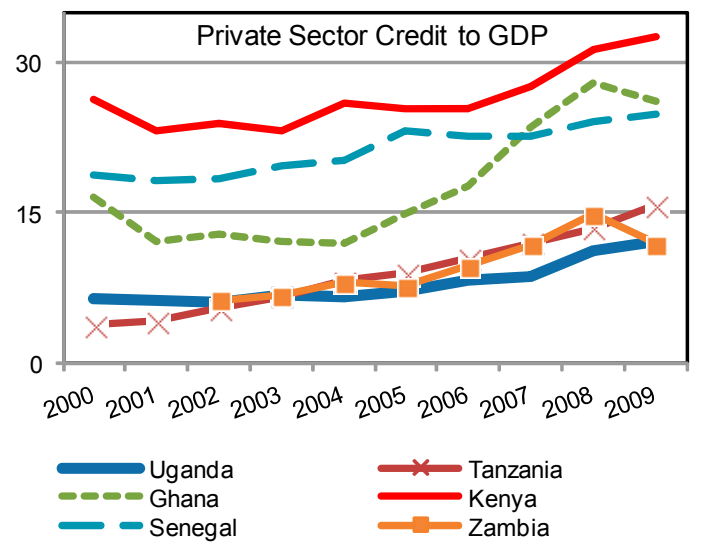

And private sector credit growth is high, but within sample range.

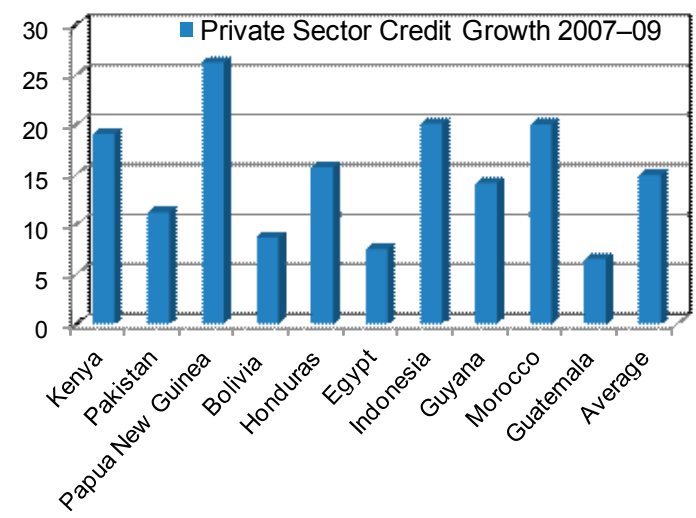

But lending rates remain sticky.

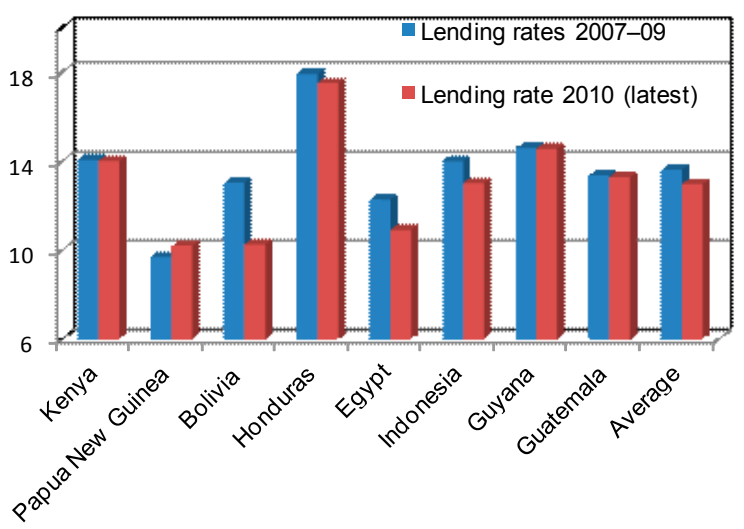

Sources: Countries' central banks; World Economic Outlook data base; and staff calculations.

1/ Except for the chart on private sector creidt to GDP, the sample comprises countries with per-capita GDP higher than Haiti and lower than China for which 2007-09 data was available, showing a 30 percentage point peak-to-through difference in private sector credit growth in 2004-09.

\section{Financial sector policy}

20. Financial supervision should adapt to new challenges associated with the modernization of the banking system, including cross-border operations and mobile banking. Specific measures include (MEFP, p. 20):

- $\quad$ Amend the Banking Act to reinforce prompt corrective action by the CBK.

- $\quad$ Adapt banking supervision to improve monitoring of financial business driven by bank agents and the use of mobile banking (Box 6). 


\section{Box 6. Kenya: Financial Sector Deepening and Modernization}

The Kenyan financial sector is the most complex and dynamic within the EAC and the third largest in SSA. Currently, 40.5 percent (7.6 million) of the adult population use the services of financial intermediaries compared to 26.4 percent in 2006.

The mobile-banking revolution and the introduction of "agency banking" have deepened financial intermediation:

- In the first half of 2010, mobile banking transfers (mainly through Equity Bank's $M$ pesa) increased by about US\$115 million, equivalent to about 10 percent of currency in circulation.

- Launched in May 2010, M-kesho (mobilephone based deposit accounts) broadens the range of money services and the potential mobilization of resources, further reducing the need to hold cash.

- Enabling third parties or "agents" to conduct banking activities on behalf of financial institutions will further reduce the barriers to financial penetration and the cost of credit.

This deepening has implications for the stability of monetary aggregates: Broad money growth doubled in 2006-10 relative to 2001-05; while increased use of mobile-based banking contributed to a significant increase in the money multiplier in the same period.

Prudential regulations need to be strengthened to cope with evaluation and monitoring of the new sources of risk. There is also a real risk that increased liquidity in the banking system may fuel credit growth further.


- Upgrade the crisis management framework taking into consideration the expansion of cross-border banking activities, in line with Financial Sector Assessment Program (FSAP) recommendations.

- Implement consolidated supervision and assess conglomeration risk of relevant local groups.

21. The financial supervisory and regulatory framework will be made more open and transparent. Main actions include the following: 
- $\quad$ Strengthen capital markets by demutualizing the stock market, in line with Monetary and Capital Market Department (MCM) recommendations.

- $\quad$ Enforce compliance of Retirement Benefit Authority (RBA) regulations on the insolvent National Social Security Fund (NSSF), in particular the appointment of external fund managers and external custodians.

- Fully implement the Crime and Anti-Money Laundering Act of 2009, including bylaws and regulations.

- $\quad$ Modify the CBK Act to allow the central bank to share information obtained from surveillance of authorized foreign exchange bureaus for criminal investigations.

22. The CBK will actively pursue to expand opportunities for small enterprises to access credit at affordable interest rates. Measures to be considered (MEFP, pp. 21, 22) are as follows:

- $\quad$ Increase the information coverage by the Credit Reference Bureau.

- Implement supervision of Savings and Credit Cooperatives (SACCOs), to help improve consumers' confidence.

- $\quad$ Simplify the collateral regime, reduce the number of stamp duties; create a unique registry and promote the use of alternative risk mitigators.

\section{Program Modalities AND Risks}

23. Program modalities. The ECF arrangement will cover the period January 2011-14. This will provide the Kenyan authorities enough time for implementing key structural reforms to strengthen the external and the fiscal position and establishing their credibility with international markets. The arrangement would include performance criteria and structural benchmarks in the fiscal, monetary and financial policy areas, with emphasis on public finance management reform. A list of benchmarks and performance criteria are included in Annexes 1 and 2.

24. Access. Staff estimate external financing needs of US\$1.4 billion over 2011-13 to facilitate foreign exchange reserves buildup while preventing an abrupt adjustment in domestic demand. Reflecting these needs and the strength of envisaged reforms, in particular those allowing for fiscal consolidation over the medium term, staff propose access of 120 percent of quota (SDR 325.7 million), which is the access norm for countries with outstanding concessional credit of less than 100 percent of quota. The programmed level of reserves would provide adequate financial buffers to withstand adverse shocks. 
25. Phasing. The proposed access will be split over three years. SDR 65.1 million (24 percent of quota) would be made available upon approval. Two additional disbursements of SDR 43.42 million (16 percent of quota) each, would be made during the first year of the program, following the March 31, 2011 and the June 30, 2011 reviews. In the subsequent years, disbursements would be made in equal tranches, following completion of semi-annual reviews. Such a schedule would support the envisaged pace of reserves buildup.

26. Financing. The program is fully financed. Of the original estimate of US\$1.4 billion financing gap, the Fund will finance US\$500 million under the proposed 120 percent of quota access. The remainder is reflected in the projected increase in grants and loan disbursements from both multilateral and bilateral donors. In particular, the World Bank is expected to disburse Development Policy Loans to support the budget in the amount of US\$300 million over the next three years. Additional budget support from the EU and investment financing from the AfDB is being discussed. Bilateral donors are expected to increase their financing, chiefly Japan and Agence Francaise de Development.

27. Poverty reduction. The government will continue to protect priority social expenditure to ensure progress in achieving social objectives as elaborated in the social pillar of the Vision 2030 Medium Term Plan, to consolidate gains in school attendance, vaccination rates, and access to improved water sources. The government will also undertake measures to mitigate the poverty impact of food insecurity on the vulnerable population (MEFP, p. 15). Priority spending including cash transfers to the vulnerable population, antiretroviral treatment expenditures, and free primary and secondary education, will be protected. A new household survey planned for 2011/12 will update information on poverty, measured at 47 percent based on the national poverty line and 20 percent for extreme poverty, based on the 2005/06 household consumption survey.

\section{The main risks to the program are:}

- a deterioration of the political outlook in the run up to elections in 2012 and during the implementation of fiscal decentralization, which could weaken political support for the program;

- a sharper-than-anticipated deterioration of the terms of trade and further weakening of the global economy, affecting the demand for nontraditional exports;

- $\quad$ a sudden reversal of the benign weather conditions in 2010 , leading to stronger pressures on the external position; and

- $\quad$ limited scope to relax fiscal policy in the event of adverse shocks given the high fiscal deficit and the gradual pace of its reduction in the baseline scenario.

29. Capacity to repay the Fund. Kenya has a strong capacity to repay the Fund. Its current obligations to the Fund are low (SDR 273.2 million at end-November 2010), the 
proposed access would not affect significantly the level of total debt (47 percent of GDP), and the risk of debt distress is low according to the most recent Debt Sustainability Analysis (DSA) (see Table 7).

30. Safeguards. An update of the safeguards assessment of the CBK that was completed on November 2, 2009, including an evaluation of the implementation of its recommendations, will take place by the time of the first review.

\section{Staff Appraisal}

31. Kenya now has the chance to become a dynamic, fast-growing economy. To seize this opportunity, the authorities need to build momentum in their reform efforts and create the conditions for a lasting acceleration in economic growth. The main macroeconomic challenge is to lower the country's external and fiscal vulnerabilities while devoting sufficient resources to implement the new constitution, and to raise infrastructure investment. By enhancing social cohesion, the new constitution could help solve many of the underlying issues behind the social and political strife that has hampered Kenya's growth performance. Higher infrastructure investment will remove key hurdles to growth and help the country to deal with global warming by increasing geothermal power capacity.

32. The policy buffers that were used in response to droughts, the 2007 social and political turmoil, and the global downturn need to be rebuilt to sustain higher growth over the medium term. Fund support will ease balance-of-payment constraints in the short term, but fiscal consolidation is needed to increase domestic savings and address the country's protracted balance of payments needs. The program envisages (a) a buildup in international reserves that should help to deal with unforeseen shocks; and (b) a gradual improvement in the fiscal position that should leave sufficient scope for pro-growth spending.

33. Despite the recent improvements, weak governance remains a chief impediment to growth. The program seeks to reduce the scope for corruption by increasing accountability in PFM. The implementation of the new constitution provides the opportunity to pass legislation that could help overhaul the PFM system, and allow stricter expenditure control by establishing a single Treasury account. Moreover, the authorities are working with the WB in the context of a Development Policy Loan (DPL) that would address governance issues as a key component of the government's strategy to improve the business climate.

\section{Kenya has made significant strides in domestic revenue mobilization, but} redesigning the tax structure is a key priority. Revamping the VAT legislation would further enhance tax collection by broadening its base. The authorities' intention to reduce income-tax exemptions would not only boost revenue, but would also improve tax-payers perception of the fairness of the tax system and thus benefit compliance. 


\section{The new constitution envisages a substantial decentralization of fiscal}

responsibilities that may improve service delivery. However, decentralization must not compromise sound fiscal management principles. It is encouraging that the authorities have already started to use Fund technical assistance in designing an effective decentralization strategy.

36. Monetary policy needs to anchor inflation expectations. The deepening of Kenya's financial system trough rapid financial inclusion has lowered the income velocity of money. Thus, inflation has declined despite abundant liquidity. In fact, the downward shift of the yield curve suggests lower inflation expectations. Nevertheless, the lagged impact of money growth on prices remains uncertain. Thus, the CBK should monitor developments closely and stand ready to tighten liquidity conditions, if signs of inflationary pressures materialize. Looking forward, the CBK should consider adopting a formal inflation targeting framework, and to this end further develop its inflation forecasting capacity.

\section{The mobile banking revolution has benefited Kenya's financial system, but it} poses challenges. The $\mathrm{CBK}$ needs to adapt banking supervision to the changing financial landscape and respond to the challenges deriving from both financial inclusion and growing regionalization of the banking system. Banking soundness indicators have continued to improve, but it will be important to avoid that the rapid credit growth associated with increasing financial inclusion translates into lower quality of banks' loan portfolio.

\section{Risks to the program are present, but the possible benefits from its successful} implementation largely outweigh them. A new flare up in social tensions cannot be ruled out, but its likelihood has declined following the approval of the new constitution. The improved political climate has opened a window of opportunity for pushing forward economic reforms that could radically transform the Kenyan economy. Fund support will help the authorities to seize this opportunity and to mobilize support from developments partners. Staff recommends approval of the authorities' request for an ECF arrangement. 
Table 1. Kenya: Selected Economic Indicators, 2008/09-2013/2014 1/

\begin{tabular}{|c|c|c|c|c|c|c|}
\hline & \multirow{2}{*}{$\begin{array}{r}\text { Actual } \\
2008 / 09\end{array}$} & \multirow{2}{*}{$\begin{array}{c}\text { Estimate } \\
2009 / 10\end{array}$} & \multicolumn{4}{|c|}{ Projections } \\
\hline & & & $2010 / 11$ & $2011 / 12$ & $2012 / 13$ & $2013 / 14$ \\
\hline & \multicolumn{6}{|c|}{ (Annual percentage change; unless otherwise indicated) } \\
\hline \multicolumn{7}{|l|}{ National accounts and prices } \\
\hline Nominal GDP (market prices, in billions of Kenya shillings) & 2,176 & 2,410 & 2,713 & 3,075 & 3,464 & 3,880 \\
\hline Real GDP growth (market prices) & 2.1 & 3.8 & 5.4 & 6.1 & 6.7 & 6.8 \\
\hline GDP deflator (average) 2/ & 9.3 & 6.7 & 6.9 & 6.8 & 5.6 & 4.9 \\
\hline Consumer price index (annual average) $2 /$ & 15.0 & 5.3 & 3.4 & 5.0 & 5.0 & 5.0 \\
\hline Consumer price index (end of period) $2 /$ & 8.6 & 3.5 & 4.0 & 5.0 & 5.0 & 5.0 \\
\hline Import volume growth, goods, and services & 13.6 & 8.7 & 5.5 & 5.8 & 6.6 & 7.2 \\
\hline Import value growth, goods, and services & 9.5 & 2.9 & 12.5 & 9.1 & 12.0 & 7.8 \\
\hline Export volume growth, goods, and services & -1.5 & 5.9 & 11.0 & 10.5 & 9.7 & 10.5 \\
\hline Export value growth, goods, and services & 0.5 & 12.8 & 8.8 & 5.6 & 15.5 & 8.3 \\
\hline Terms of trade, goods, and services (Base year 2000) & 6.5 & 12.1 & -7.3 & -7.4 & 0.2 & -2.7 \\
\hline Ksh per US $\$$ exchange rate (end of period) $3 /$ & 77.2 & 81.9 & 81.0 & & & \\
\hline Nominal effective exchange rate (- depreciation; end of period) & -10.4 & -1.2 & $\ldots$ & $\ldots$ & $\ldots$ & $\ldots$ \\
\hline Real effective exchange rate (- depreciation; end of period) & -4.3 & -2.4 & $\cdots$ & $\cdots$ & $\cdots$ & $\cdots$ \\
\hline \multicolumn{7}{|l|}{ Money and credit } \\
\hline \multirow[t]{2}{*}{ M3 (broad money and foreign currency deposits, end period) } & 13.0 & 26.2 & 19.2 & 16.6 & 14.1 & 14.7 \\
\hline & \multicolumn{6}{|c|}{ (In percent of GDP; unless otherwise indicated) } \\
\hline \multicolumn{7}{|l|}{ Investment and saving } \\
\hline Investment & 19.9 & 20.5 & 23.1 & 24.5 & 25.2 & 25.2 \\
\hline Central government & 7.5 & 8.9 & 10.3 & 10.6 & 11.1 & 10.9 \\
\hline Other & 12.4 & 11.6 & 12.8 & 13.9 & 14.1 & 14.3 \\
\hline Gross national saving & 11.9 & 15.1 & 15.8 & 17.0 & 18.7 & 20.2 \\
\hline Central government 4/ & 2.2 & 1.6 & 2.7 & 3.7 & 5.1 & 5.6 \\
\hline Other & 9.7 & 13.6 & 13.1 & 13.3 & 13.7 & 14.6 \\
\hline \multicolumn{7}{|l|}{ Central government budget 5 / } \\
\hline Total revenue & 22.4 & 22.7 & 25.2 & 25.3 & 25.3 & 25.6 \\
\hline Total expenditure and net lending & 27.8 & 30.1 & 32.9 & 31.8 & 31.2 & 30.8 \\
\hline Overall balance (commitment basis) excluding grants & -5.4 & -7.4 & -7.7 & -6.6 & -5.9 & -5.2 \\
\hline Overall balance (commitment basis) including grants & -4.5 & -6.5 & -6.5 & -5.3 & -4.6 & -3.9 \\
\hline Primary budget balance & -1.5 & -3.9 & -3.8 & -2.7 & -1.9 & -1.2 \\
\hline Net domestic borrowing & 3.1 & 5.5 & 4.6 & 3.2 & 1.3 & 1.2 \\
\hline \multicolumn{7}{|l|}{ Balance of payments } \\
\hline Exports value, goods, and services & 26.6 & 27.3 & 27.9 & 26.0 & 26.6 & 25.8 \\
\hline Imports value, goods, and services & 42.0 & 39.4 & 41.6 & 40.0 & 39.8 & 38.2 \\
\hline Current external balance, including official transfers & -8.0 & -5.3 & -7.3 & -7.5 & -6.5 & -5.0 \\
\hline Current external balance, excluding official transfers & -7.9 & -5.3 & -7.2 & -7.5 & -6.4 & -5.0 \\
\hline \multicolumn{7}{|l|}{ Gross international reserve coverage } \\
\hline In billions of U.S. dollars (end of period) & 3.2 & 3.8 & 4.2 & 4.6 & 5.9 & 7.4 \\
\hline In months of next year imports (end of period) & 3.1 & 3.3 & 3.3 & 3.3 & 3.8 & 4.4 \\
\hline \multicolumn{7}{|l|}{ Public debt 6/ } \\
\hline Total public debt, net (percent of GDP) & 41.7 & 45.7 & 47.1 & 46.9 & 45.1 & 43.6 \\
\hline Of which: external debt & 23.3 & 23.5 & 22.8 & 22.2 & 21.9 & 21.7 \\
\hline Domestic debt, net of deposits & 18.5 & 22.2 & 24.3 & 24.6 & 23.2 & 21.9 \\
\hline
\end{tabular}

Sources: Kenyan authorities; and IMF staff estimates and projections.

$1 /$ Fiscal year is from July 1st through June 30th.

2/ The CPI series was revised in November 2009 based on a new methodology.

3/ Actual as of June, 2010.

4/ Revenue plus program grants minus recurrent expenditure.

5/ Coverage increased in FY 2010/11 to incorporate tuitions and fees to universities and hospitals, and associated expenditure.

6/ Refers to the debt of the central government. 
Table 2a. Kenya: Central Government Financial Operations 1/

\begin{tabular}{|c|c|c|c|c|c|c|}
\hline & \multicolumn{2}{|c|}{ Estimate } & \multicolumn{4}{|c|}{ Projections } \\
\hline & $2008 / 09$ & $2009 / 10$ & $2010 / 11$ & $2011 / 12$ & $2012 / 13$ & $2013 / 14$ \\
\hline Revenues and grants & 507.6 & 568.8 & 716.5 & 815.0 & 920.4 & $1,043.9$ \\
\hline Revenue & 487.9 & 548.1 & 684.2 & 777.3 & 876.2 & 992.3 \\
\hline Tax revenue & 417.4 & 466.5 & 555.5 & 635.6 & 720.9 & 818.7 \\
\hline Income tax & 184.4 & 209.1 & 252.0 & 288.4 & 326.0 & 367.0 \\
\hline Import duty (net) & 36.2 & 41.3 & 48.6 & 54.2 & 57.8 & 67.0 \\
\hline Excise duty & 69.9 & 74.1 & 86.2 & 98.8 & 111.7 & 125.2 \\
\hline Value-added tax & 126.9 & 142.0 & 168.7 & 194.3 & 225.4 & 259.6 \\
\hline Nontax revenue & 68.2 & 81.6 & 128.7 & 141.7 & 155.3 & 173.6 \\
\hline Investment income & 6.9 & 8.4 & 11.9 & 13.5 & 15.2 & 17.0 \\
\hline Other & 31.5 & 32.7 & 36.8 & 41.7 & 46.9 & 52.6 \\
\hline Local Authorities Trust Fund (LATF) & 9.2 & 0.0 & 13.4 & 15.2 & 17.2 & 19.3 \\
\hline Ministerial and departmental fees (Appropriations in Aid-AIA) 2/ & 20.6 & 40.6 & 66.6 & 71.4 & 76.1 & 84.7 \\
\hline Grants & 19.7 & 20.7 & 32.3 & 37.8 & 44.2 & 51.6 \\
\hline Food/debt relief grants 3 / & 0.0 & 0.0 & 0.0 & 0.0 & 0.0 & 0.0 \\
\hline Project grants & 19.7 & 20.7 & 32.3 & 37.8 & 44.2 & 51.6 \\
\hline Program grants & 0.0 & 0.0 & 0.0 & 0.0 & 0.0 & 0.0 \\
\hline Expenditure and net lending & 601.8 & 725.2 & 892.3 & 978.7 & $1,081.4$ & $1,194.6$ \\
\hline Recurrent expenditure & 435.5 & 504.3 & 602.3 & 637.4 & 688.3 & 765.6 \\
\hline Interest payments & 47.3 & 63.5 & 74.9 & 82.0 & 94.7 & 104.9 \\
\hline Domestic interest & 41.2 & 57.4 & 67.9 & 73.3 & 82.5 & 89.6 \\
\hline Foreign interest due & 6.1 & 6.1 & 7.0 & 8.6 & 12.2 & 15.3 \\
\hline Wages and benefits (civil service) & 155.2 & 172.6 & 197.7 & 218.4 & 247.1 & 274.3 \\
\hline Civil service reform & 0.1 & 0.0 & 0.1 & 0.2 & 0.2 & 0.2 \\
\hline Pensions, etc. & 27.2 & 29.0 & 28.6 & 40.5 & 44.8 & 49.5 \\
\hline Other 2/ & 159.7 & 182.3 & 237.4 & 238.6 & 242.7 & 276.7 \\
\hline Defense and National Security Intelligence Service (NSIS) & 0.0 & 56.9 & 63.7 & 57.7 & 58.8 & 60.0 \\
\hline Development and net lending & 166.3 & 214.7 & 281.9 & 327.8 & 387.1 & 423.8 \\
\hline Domestically financed & 109.6 & 151.9 & 174.2 & 203.1 & 207.7 & 272.0 \\
\hline Foreign financed & 54.3 & 61.6 & 105.4 & 122.2 & 176.8 & 151.4 \\
\hline Net lending & 2.4 & 1.2 & 2.4 & 2.5 & 2.6 & 0.4 \\
\hline Civil Contingency Fund & 0.0 & 0.0 & 2.0 & 2.0 & 0.0 & 0.0 \\
\hline Expenditure measures & 0.0 & 0.0 & 0.0 & 0.0 & 0.0 & 0.0 \\
\hline Drought expenditures & 0.0 & 6.2 & 2.0 & 2.0 & 3.0 & 3.2 \\
\hline Constitutional Reform 4/ & 0.0 & 0.0 & 4.0 & 9.5 & 3.0 & 2.0 \\
\hline Balance (com mitment bas is, excluding grants) & -113.9 & -177.1 & -208.1 & -201.4 & -205.2 & -202.2 \\
\hline Balance (commitment bas is, including grants) & -94.2 & -156.4 & -175.8 & -163.7 & -161.0 & -150.6 \\
\hline Adjustments to cas $h$ bas is & 12.8 & 0.0 & -1.1 & 0.0 & 0.0 & 0.0 \\
\hline Balance (cas $\mathrm{h}$ bas is, including grants) & -81.5 & -156.4 & -176.9 & -163.7 & -161.0 & -150.6 \\
\hline Financing & 88.7 & 156.4 & 176.9 & 163.7 & 161.0 & 150.6 \\
\hline Net foreign financing & 16.8 & 22.9 & 51.9 & 66.5 & 115.6 & 102.9 \\
\hline Project loans & 32.3 & 39.8 & 71.9 & 83.0 & 91.8 & 104.7 \\
\hline Program loans & 0.0 & 0.0 & 0.0 & 8.1 & 8.1 & 8.1 \\
\hline Commercial borrow ing $5 /$ & 0.0 & 0.0 & 0.0 & 0.0 & 40.8 & 19.4 \\
\hline Repayments due & -17.5 & -18.7 & -20.5 & -24.6 & -25.1 & -29.3 \\
\hline Change in arrears & 1.5 & 1.4 & 0.0 & 0.0 & 0.0 & 0.0 \\
\hline Rescheduling/debt sw ap & 0.5 & 0.5 & 0.5 & 0.0 & 0.0 & 0.0 \\
\hline Net domestic financing & 69.4 & 133.5 & 125.0 & 97.2 & 45.4 & 47.7 \\
\hline Financing gap (stat. dis crepancy for outturns) & -7.2 & 0.0 & 0.0 & 0.0 & 0.0 & 0.0 \\
\hline \multicolumn{7}{|l|}{ Memorandum items: } \\
\hline Nominal GDP & $2,175.6$ & $2,409.6$ & $2,713.3$ & $3,074.7$ & $3,463.6$ & $3,880.1$ \\
\hline Primary budget balance including grants & -34.2 & -92.9 & -102.1 & -81.7 & -66.3 & -45.7 \\
\hline Primary budget balance including grants, excluding concessional & & & & & & \\
\hline project financing (above the line, cash) & -1.9 & -53.1 & -30.2 & 1.3 & 25.5 & 59.0 \\
\hline \multicolumn{7}{|l|}{ Primary budget balance including grants, excluding concessional } \\
\hline project financing (below the line) & -6.7 & -53.1 & -30.2 & 1.3 & 25.5 & 59.0 \\
\hline Stock of domestic debt, net (end of period) 6/ & 401.5 & 534.8 & 659.8 & 757.0 & 802.5 & 850.2 \\
\hline Net present value (NPV) of total public debt, net & 694.4 & 913.5 & $1,097.3$ & $1,251.6$ & $1,367.5$ & $1,476.9$ \\
\hline Total public debt, net of deposits & 907.7 & $1,100.4$ & $1,277.3$ & $1,441.0$ & $1,561.2$ & $1,692.5$ \\
\hline End-of-period exchange rate Ksh/US\$ & 77.2 & 81.9 & 81.0 & 81.0 & 81.0 & 81.0 \\
\hline
\end{tabular}

Sources: Kenyan authorities; and IMF staff estimates and projections.

$1 /$ Fiscal year runs from July to June.

2/ Coverage increased in FY 2010/11 to incorporate tuitions and fees to universities and hospitals, and associated expenditure.

$3 /$ Includes debt relief from a debt sw ap deal w ith Italy.

4/ Includes estimated expenditures associated $w$ ith the implementation of the new constitution.

$5 /$ Includes planned sovereign bonds.

6/ Refers to the debt of the central government. 
Table 2b. Kenya: Central Government Financial Operations 1/

\begin{tabular}{|c|c|c|c|c|c|}
\hline \multicolumn{2}{|c|}{ Estimate } & \multicolumn{4}{|c|}{ Projections } \\
\hline $2008 / 09$ & $2009 / 10$ & $2010 / 11$ & $2011 / 12$ & $2012 / 13$ & $2013 / 14$ \\
\hline 22.4 & 22.7 & 25.2 & 25.3 & 25.3 & 25.6 \\
\hline 19.2 & 19.4 & 20.5 & 20.7 & 20.8 & 21.1 \\
\hline 8.5 & 8.7 & 9.3 & 9.4 & 9.4 & 9.5 \\
\hline 5.8 & 5.9 & 6.2 & 6.3 & 6.5 & 6.7 \\
\hline 3.1 & 3.4 & 4.7 & 4.6 & 4.5 & 4.5 \\
\hline 0.3 & 0.3 & 0.4 & 0.4 & 0.4 & 0.4 \\
\hline 1.4 & 1.4 & 1.4 & 1.4 & 1.4 & 1.4 \\
\hline 0.4 & 0.0 & 0.5 & 0.5 & 0.5 & 0.5 \\
\hline 0.0 & 0.0 & 0.0 & 0.0 & 0.0 & 0.0 \\
\hline 27.7 & 30.1 & 32.9 & 31.8 & 31.2 & 30.8 \\
\hline 20.0 & 20.9 & 22.2 & 20.7 & 19.9 & 19.7 \\
\hline 2.2 & 2.6 & 2.8 & 2.7 & 2.7 & 2.7 \\
\hline 1.9 & 2.4 & 2.5 & 2.4 & 2.4 & 2.3 \\
\hline 0.3 & 0.3 & 0.3 & 0.3 & 0.4 & 0.4 \\
\hline 7.1 & 7.2 & 7.3 & 7.1 & 7.1 & 7.1 \\
\hline 0.0 & 0.0 & 0.0 & 0.0 & 0.0 & 0.0 \\
\hline 1.2 & 1.2 & 1.1 & 1.3 & 1.3 & 1.3 \\
\hline 7.3 & 7.6 & 8.8 & 7.8 & 7.0 & 7.1 \\
\hline 0.0 & 2.4 & 2.3 & 1.9 & 1.7 & 1.5 \\
\hline 0.0 & 0.0 & 0.1 & 0.3 & 0.1 & 0.1 \\
\hline-5.2 & -7.4 & -7.7 & -6.6 & -5.9 & -5.2 \\
\hline-4.3 & -6.5 & -6.5 & -5.3 & -4.6 & -3.9 \\
\hline 0.6 & 0.0 & 0.0 & 0.0 & 0.0 & 0.0 \\
\hline-3.7 & -6.5 & -6.5 & -5.3 & -4.6 & -3.9 \\
\hline 4.1 & 6.5 & 6.5 & 5.3 & 4.6 & 3.9 \\
\hline 0.8 & 1.0 & 1.9 & 2.2 & 3.3 & 2.7 \\
\hline 1.5 & 1.7 & 2.7 & 2.7 & 2.7 & 2.7 \\
\hline 0.0 & 0.0 & 0.0 & 0.3 & 0.2 & 0.2 \\
\hline 0.0 & 0.0 & 0.0 & 0.0 & 1.2 & 0.5 \\
\hline-0.8 & -0.8 & -0.8 & -0.8 & -0.7 & -0.8 \\
\hline 0.1 & 0.1 & 0.0 & 0.0 & 0.0 & 0.0 \\
\hline 0.0 & 0.0 & 0.0 & 0.0 & 0.0 & 0.0 \\
\hline 3.2 & 5.5 & 4.6 & 3.2 & 1.3 & 1.2 \\
\hline-0.3 & 0.0 & 0.0 & 0.0 & 0.0 & 0.0 \\
\hline \multicolumn{6}{|c|}{ Memorandum items: } \\
\hline $2,175.6$ & $2,409.6$ & $2,713.3$ & $3,074.7$ & $3,463.6$ & $3,880.1$ \\
\hline-1.6 & -3.9 & -3.8 & -2.7 & -1.9 & -1.2 \\
\hline 18.5 & 22.2 & 24.3 & 24.6 & 23.2 & 21.9 \\
\hline 31.9 & 37.9 & 40.4 & 40.7 & 39.5 & 38.1 \\
\hline
\end{tabular}

Sources: Kenyan authorities; and IMF staff estimates and projections.

$1 /$ Fiscal year runs from July to June.

2/ Coverage increased in FY 2010/11 to incorporate tuitions and fees to universities and hospitals, and associated expenditı

3 / Includes debt relief from a debt sw ap deal w ith Italy.

$4 /$ Includes estimated expenditures associated $w$ ith the implementation of the new constitution.

$5 /$ Includes planned sovereign bonds.

6/ Refers to the debt of the central government. 
Table 3. Kenya: Monetary Survey December 08-December 11

(In billions of Kenyan shillings, unless otherw ise indicated)

\begin{tabular}{|c|c|c|c|c|c|c|c|c|c|}
\hline & Dec-08 & Dec-09 & Jun-10 & Sep-10 & Dec-10 & Mar-11 & Jun-11 & Sep-11 & Dec-11 \\
\hline \multicolumn{10}{|l|}{ Central Bank of Kenya } \\
\hline Net foreign assets & 199.1 & 222.8 & 242.9 & 257.7 & 263.5 & 261.7 & 264.1 & 267.6 & 281.5 \\
\hline (In millions of U.S. dollars) $1 /$ & $2,560.7$ & $2,960.8$ & $2,967.1$ & $3,197.1$ & $3,265.2$ & $3,230.6$ & $3,260.0$ & $3,303.8$ & $3,474.8$ \\
\hline Net domestic assets & -35.5 & -40.8 & -32.6 & -47.8 & -47.8 & -47.5 & -47.5 & -46.9 & -29.1 \\
\hline Net domestic credit & -1.5 & -6.3 & 10.3 & 2.3 & -1.3 & -6.8 & -6.8 & -6.2 & 11.6 \\
\hline Government (net) & 0.0 & -25.5 & 7.5 & -0.6 & -0.6 & -2.0 & -2.0 & -2.0 & -2.0 \\
\hline Commercial banks (net) & -4.0 & 16.5 & 0.0 & 0.0 & -0.7 & -4.8 & -4.8 & -4.2 & 13.6 \\
\hline Other items (net) & -34.0 & -34.5 & -42.9 & -50.1 & -46.5 & -40.7 & -40.7 & -40.7 & -40.7 \\
\hline Reserve money & 163.6 & 182.0 & 210.3 & 209.9 & 215.7 & 214.1 & 216.6 & 220.7 & 252.4 \\
\hline Currency outside banks & 93.7 & 100.9 & 101.3 & 104.7 & 117.0 & 107.6 & 106.4 & 111.0 & 136.1 \\
\hline Bank reserves & 69.9 & 81.1 & 109.0 & 105.2 & 98.7 & 106.6 & 110.2 & 109.6 & 116.3 \\
\hline \multicolumn{10}{|l|}{ Banks } \\
\hline Net foreign assets & 50.4 & 21.0 & 37.4 & 15.0 & 37.1 & 35.4 & 31.2 & 29.7 & 33.4 \\
\hline (In millions of U.S. dollars) & 648.1 & 276.7 & 456.5 & 185.6 & 460.2 & 437.4 & 384.9 & 367.0 & 412.4 \\
\hline Reserves & 69.9 & 81.1 & 109.0 & 105.2 & 98.7 & 106.6 & 110.2 & 109.6 & 116.3 \\
\hline Credit to CBK & 4.0 & -16.5 & 0.0 & 0.0 & 0.7 & 4.8 & 4.8 & 4.2 & -13.6 \\
\hline Net domestic assets & 673.7 & 850.4 & 940.6 & $1,002.8$ & $1,067.3$ & $1,106.9$ & $1,149.8$ & $1,227.0$ & $1,300.1$ \\
\hline Net domestic credit & 813.1 & 978.6 & $1,076.5$ & $1,136.8$ & $1,206.1$ & $1,248.4$ & $1,287.4$ & $1,365.0$ & $1,439.0$ \\
\hline Government (net) & 155.3 & 230.5 & 270.2 & 278.9 & 305.7 & 339.5 & 336.4 & 364.6 & 381.9 \\
\hline Other public sector & 11.8 & 12.5 & 9.9 & 17.9 & 13.6 & 11.4 & 9.2 & 16.8 & 13.3 \\
\hline Private sector & 645.9 & 735.6 & 796.4 & 840.0 & 886.8 & 897.5 & 941.8 & 983.6 & $1,043.8$ \\
\hline Other items (net) & -139.4 & -128.2 & -135.9 & -134.0 & -138.8 & -141.5 & -137.6 & -138.0 & -139.0 \\
\hline Total deposits & 797.9 & 935.9 & $1,086.9$ & $1,123.0$ & $1,203.8$ & $1,253.8$ & $1,295.9$ & $1,370.6$ & $1,436.2$ \\
\hline \multicolumn{10}{|l|}{ Monetary survey } \\
\hline Net foreign assets & 249.5 & 243.8 & 280.3 & 272.7 & 300.6 & 297.1 & 295.2 & 297.3 & 314.9 \\
\hline (In millions of U.S. dollars) & $3,208.8$ & $3,237.5$ & $3,423.6$ & $3,382.7$ & $3,725.4$ & $3,668.0$ & $3,644.9$ & $3,670.8$ & $3,887.2$ \\
\hline Net domestic assets & 651.6 & 801.9 & 918.7 & 970.9 & $1,027.2$ & $1,091.3$ & $1,134.2$ & $1,220.5$ & $1,269.3$ \\
\hline Net domestic credit & 815.5 & 955.8 & $1,086.8$ & $1,139.1$ & $1,208.0$ & $1,248.9$ & $1,287.9$ & $1,365.5$ & $1,439.5$ \\
\hline Government (net) & 155.3 & 205.1 & 277.7 & 278.2 & 305.1 & 337.5 & 334.4 & 362.6 & 379.9 \\
\hline Other public sector & 11.8 & 12.5 & 9.9 & 17.9 & 13.6 & 11.4 & 9.2 & 16.8 & 13.3 \\
\hline Private & 648.4 & 738.3 & 799.2 & 842.9 & 889.3 & 900.0 & 944.3 & 986.1 & $1,046.3$ \\
\hline Other items (net) & -164.0 & -153.9 & -168.1 & -168.2 & -180.8 & -157.6 & -153.7 & -145.0 & -170.3 \\
\hline M1 & 392.8 & 442.2 & 511.6 & 536.9 & 571.1 & 597.1 & 619.0 & 657.3 & 688.5 \\
\hline Money and quasimoney (M2) & 766.4 & 898.1 & $1,033.7$ & $1,078.3$ & $1,153.9$ & $1,206.5$ & $1,250.8$ & $1,328.1$ & $1,391.1$ \\
\hline M2 plus resident foreign currency deposits (M3) & 901.1 & $1,045.7$ & $1,198.9$ & $1,243.6$ & $1,327.9$ & $1,388.4$ & $1,429.5$ & $1,517.8$ & $1,584.2$ \\
\hline M3 plus nonbank holdings of government debt $(L)$ & $1,091.9$ & $1,280.5$ & $1,443.4$ & $1,527.4$ & $1,598.6$ & $1,671.5$ & $1,720.9$ & $1,827.3$ & $1,907.2$ \\
\hline \multicolumn{10}{|c|}{ Memorandum items (annual percent change unless otherwise specified): } \\
\hline M1 & 5.2 & 12.6 & 27.7 & 23.9 & 29.1 & 28.4 & 21.0 & 22.4 & 20.6 \\
\hline M2 & 14.9 & 17.2 & 27.3 & 27.0 & 28.5 & 25.8 & 21.0 & 23.2 & 20.6 \\
\hline M3 & 15.9 & 16.0 & 26.2 & 26.0 & 27.0 & 25.3 & 19.2 & 22.0 & 19.3 \\
\hline Deposits & 17.4 & 17.3 & 27.2 & 26.0 & 28.6 & 25.1 & 19.2 & 22.0 & 19.3 \\
\hline Reserve money & 4.2 & 11.2 & 31.5 & 28.4 & 18.5 & 20.7 & 3.0 & 5.1 & 17.0 \\
\hline Currency outside banks & -2.3 & 7.6 & 16.0 & 19.5 & 16.0 & 11.6 & 5.1 & 6.0 & 16.3 \\
\hline Net domestic credit & 24.3 & 17.2 & 25.8 & 27.9 & 26.4 & 22.8 & 18.5 & 19.9 & 19.2 \\
\hline Government (net) & 13.0 & 32.0 & 59.7 & 48.7 & 48.8 & 36.7 & 20.4 & 30.3 & 24.5 \\
\hline Private & 28.6 & 13.9 & 16.8 & 22.9 & 20.5 & 18.5 & 18.2 & 17.0 & 17.7 \\
\hline Currency outside banks & -2.3 & 7.6 & 16.0 & 19.5 & 16.0 & 11.6 & 5.1 & & \\
\hline Net domestic assets of the banking sector & 24.8 & 23.1 & 34.6 & 32.7 & 28.1 & 30.0 & 23.5 & 25.7 & 23.6 \\
\hline NDA grow th (as a percent of the base period M3) & 13.3 & 13.8 & 20.3 & 19.6 & 17.6 & 18.6 & 14.9 & 16.3 & 15.1 \\
\hline Multiplier (average M3/RM) & 5.5 & 5.9 & 5.9 & 5.9 & 6.0 & 6.1 & 6.3 & 6.5 & 6.5 \\
\hline Velocity (GDP/M2) & 2.8 & 2.7 & 2.6 & 2.5 & 2.4 & 2.4 & 2.3 & 2.3 & 2.2 \\
\hline
\end{tabular}

Sources: Kenyan authorities; and IMF staff estimates and projections.

1/ For historical data, at implicit CBK exchange rate. 
Table 4. Kenya: Balance of Payments, 2007/08-2014/15

(In millions of dollars; unless otherw ise indicated)

\begin{tabular}{|c|c|c|c|c|c|c|c|c|}
\hline & \multirow[b]{2}{*}{$2007 / 08$} & \multicolumn{2}{|c|}{ Prel. } & \multicolumn{5}{|c|}{ Projections } \\
\hline & & $2008 / 09$ & $2009 / 10$ & $2010 / 11$ & $2011 / 12$ & $2012 / 13$ & $2013 / 14$ & $2014 / 15$ \\
\hline $\begin{array}{l}\text { Current account } \\
\text { Excluding official transfers }\end{array}$ & $\begin{array}{l}-1,182.6 \\
-1,214.5\end{array}$ & $\begin{array}{l}-2,288.6 \\
-2,267.7\end{array}$ & $\begin{array}{l}-1,680.6 \\
-1,661.3\end{array}$ & $\begin{array}{l}-2,430.4 \\
-2,409.9\end{array}$ & $\begin{array}{l}-2,851.1 \\
-2,829.1\end{array}$ & $\begin{array}{l}-2,776.6 \\
-2,754.6\end{array}$ & $\begin{array}{l}-2,408.2 \\
-2,386.2\end{array}$ & $\begin{array}{l}-2,015.8 \\
-1,993.8\end{array}$ \\
\hline Exports, f.o.b. & $4,545.4$ & $4,641.6$ & $4,885.5$ & $5,395.8$ & $5,846.5$ & $6,626.4$ & $7,072.9$ & $7,633.7$ \\
\hline Coffee & 169.3 & 179.4 & 194.2 & 228.4 & 187.7 & 183.7 & 184.9 & 187.9 \\
\hline Tea & 754.8 & 876.9 & $1,127.2$ & 789.4 & 802.5 & 832.7 & 847.4 & 881.0 \\
\hline Horticulture & 739.3 & 668.2 & 714.8 & 902.2 & 939.8 & $1,047.7$ & $1,102.4$ & $1,130.6$ \\
\hline Imports, f.o.b. & $-9,139.0$ & $-10,232.8$ & $-10,480.1$ & $-11,765.3$ & $-12,802.2$ & $-14,491.7$ & $-15,735.0$ & $-17,052.5$ \\
\hline Oil & $-2,448.0$ & $-2,555.7$ & $-2,635.6$ & $-3,080.4$ & $-3,373.5$ & $-3,839.0$ & $-4,306.0$ & $-4,735.2$ \\
\hline Other private & $-6,602.1$ & $-7,540.3$ & $-7,743.1$ & $-8,537.5$ & $-9,266.4$ & $-10,483.1$ & $-11,253.5$ & $-12,135.2$ \\
\hline Of which: Special—Maize and sugar & -100.0 & -441.5 & -360.9 & -224.9 & -153.7 & -157.0 & -160.5 & -165.0 \\
\hline Balance on goods & $-4,593.6$ & $-5,591.3$ & $-5,594.6$ & $-6,369.5$ & $-6,955.6$ & $-7,865.2$ & $-8,662.1$ & $-9,418.8$ \\
\hline $\begin{array}{l}\text { Balance on services } \\
\text { Credit } \\
\text { Of which: Foreign travel credit } 1 / \\
\text { Debit }\end{array}$ & $\begin{array}{r}1,190.4 \\
3,024.8 \\
763.2 \\
-1,834.4\end{array}$ & $\begin{array}{r}1,185.0 \\
2,967.8 \\
705.8 \\
-1,782.8\end{array}$ & $\begin{array}{r}1,815.4 \\
3,701.6 \\
871.1 \\
-1,886.3\end{array}$ & $\begin{array}{r}1,804.4 \\
3,949.9 \\
1,020.6 \\
-2,145.5\end{array}$ & $\begin{array}{r}1,652.9 \\
4,021.7 \\
1,178.2 \\
-2,368.8\end{array}$ & $\begin{array}{r}2,262.0 \\
4,767.7 \\
1,395.1 \\
-2,505.8\end{array}$ & $\begin{array}{r}\mathbf{2 , 6 8 0 . 8} \\
5,265.0 \\
1,555.5 \\
-2,584.2\end{array}$ & $\begin{array}{r}3,121.3 \\
6,013.4 \\
1,822.2 \\
-2,892.1\end{array}$ \\
\hline Balance on goods and services & $-3,403.2$ & $-4,406.2$ & $-3,779.2$ & $-4,565.1$ & $-5,302.7$ & $-5,603.2$ & $-5,981.3$ & $-6,297.6$ \\
\hline Income (net) & -51.0 & -76.7 & -82.9 & -61.7 & -33.0 & 0.2 & 35.0 & 110.6 \\
\hline Credit & 192.5 & 161.6 & 197.2 & 192.6 & 235.6 & 291.0 & 361.6 & 492.8 \\
\hline Debit & -243.5 & -238.3 & -280.1 & -254.3 & -268.5 & -290.8 & -326.6 & -382.2 \\
\hline \multicolumn{9}{|l|}{ Of which: } \\
\hline Official interest payments & -86.6 & -83.2 & -79.2 & -92.6 & -106.0 & -123.9 & -151.6 & -196.6 \\
\hline Other & -156.9 & -155.1 & -200.1 & -161.7 & -162.5 & -166.9 & -175.0 & -185.6 \\
\hline Current transfers (net) & $2,271.7$ & $2,194.4$ & $2,181.5$ & $2,196.3$ & $2,484.5$ & $2,826.4$ & $3,538.1$ & $4,171.2$ \\
\hline Private (net) & $2,239.8$ & $2,215.3$ & $2,200.8$ & $2,216.9$ & $2,506.5$ & $2,848.4$ & $3,560.1$ & $4,193.2$ \\
\hline Of which: Remittances & 952.8 & 981.4 & 989.5 & $1,049.3$ & $1,220.1$ & $1,458.4$ & $1,713.6$ & $2,013.5$ \\
\hline Official (net) & 31.9 & -20.9 & -19.3 & -20.6 & -22.0 & -22.0 & -22.0 & -22.0 \\
\hline Capital and financial account & $1,881.4$ & $1,863.6$ & $2,282.5$ & $2,711.6$ & $3,157.7$ & $3,933.6$ & $3,892.2$ & $3,488.9$ \\
\hline Capital account (incl. capital transfers) & 309.9 & 258.2 & 259.8 & 399.0 & 466.0 & 545.7 & 637.0 & 637.0 \\
\hline Financial account $2 /$ & $1,571.4$ & $1,605.4$ & $2,022.8$ & $2,312.6$ & $2,691.7$ & $3,387.9$ & $3,255.1$ & $2,851.9$ \\
\hline Net foreign direct investment & 891.7 & 652.5 & 403.5 & 549.2 & 661.0 & 721.2 & 786.7 & 744.0 \\
\hline In Kenya & 994.4 & 672.0 & 431.1 & 573.2 & 685.0 & 745.2 & 810.7 & 765.0 \\
\hline Abroad & -102.7 & -19.5 & -27.6 & -24.0 & -24.0 & -24.0 & -24.0 & -21.0 \\
\hline Net Portfolio investment & -36.5 & -10.3 & -73.5 & 3.4 & 7.4 & 8.5 & 9.6 & 10.8 \\
\hline Liabilities & 6.1 & 8.9 & 9.8 & 12.9 & 16.6 & 17.8 & 19.2 & 20.6 \\
\hline Assets & -42.6 & -19.2 & -83.3 & -9.5 & -9.2 & -9.4 & -9.6 & -9.8 \\
\hline Net other investment & 716.3 & 614.8 & $1,349.8$ & $1,760.0$ & $2,023.2$ & $2,658.2$ & $2,458.8$ & $2,097.1$ \\
\hline Official, medium, and long term & 94.9 & 204.9 & 624.1 & 639.2 & 874.6 & $1,449.8$ & $1,339.8$ & $1,195.7$ \\
\hline Inflows & 323.0 & 417.3 & 844.5 & 888.2 & $1,124.9$ & $1,733.3$ & $1,628.6$ & $1,497.8$ \\
\hline Outflow s & -228.1 & -212.4 & -220.3 & -248.9 & -250.3 & -283.6 & -288.8 & -302.1 \\
\hline Private, medium, and long term & 25.6 & -23.9 & -54.8 & -30.1 & 95.6 & 358.4 & 269.0 & 51.3 \\
\hline Energy financing & 48.7 & 65.3 & 38.1 & 55.0 & 67.9 & 73.1 & 49.7 & 16.8 \\
\hline Kenya Airw ays & -5.9 & -76.5 & -46.2 & -65.4 & 27.7 & 285.3 & 219.3 & 34.5 \\
\hline Other & -17.2 & -12.7 & -46.7 & -19.7 & 0.0 & 0.0 & 0.0 & 0.0 \\
\hline Short-term capital & 322.2 & 433.8 & 780.5 & $1,150.8$ & $1,053.0$ & 850.0 & 850.0 & 850.0 \\
\hline Errors and omissions & 273.6 & 348.5 & 342.9 & 0.0 & 0.0 & 0.0 & 0.0 & 0.0 \\
\hline Overall balance & 698.8 & -425.0 & 601.9 & 281.2 & 306.6 & $1,157.0$ & $1,484.0$ & $1,473.1$ \\
\hline Financing items & -698.8 & 425.0 & -601.9 & -281.2 & -306.6 & $-1,157.0$ & $-1,484.0$ & $-1,473.1$ \\
\hline Reserve assets (gross) & -719.8 & 224.0 & -579.6 & -354.6 & -474.0 & $-1,244.4$ & $-1,491.9$ & $-1,402.0$ \\
\hline Use of Fund credit and loans to the Fund (net) & 47.0 & 195.1 & -22.4 & -25.3 & -35.8 & -44.2 & -57.8 & -71.1 \\
\hline Disbursements & 57.4 & 209.5 & 0.0 & 0.0 & 0.0 & 0.0 & 0.0 & 0.0 \\
\hline Repayments & -10.5 & -14.4 & -22.4 & -25.3 & -35.8 & -44.2 & -57.8 & -71.1 \\
\hline Change in arrears & -25.9 & 0.0 & 0.0 & 0.0 & 0.0 & 0.0 & 0.0 & 0.0 \\
\hline Rescheduling/debt su & 0.0 & 5.9 & 0.0 & 0.0 & 6.0 & 0.0 & 0.0 & 0.0 \\
\hline Proposed IMF ECF financing & 0.0 & 0.0 & 0.0 & 99 & 197 & 132 & 66 & 0 \\
\hline \multicolumn{9}{|l|}{ Memorandum items: } \\
\hline Gross official reserves (end of period) & 3,443 & 3,219 & 3,799 & 4,153 & 4,627 & 5,872 & 7,363 & 8,765 \\
\hline (In months of follow ing year's imports of goods and services) & 3.4 & 3.1 & 3.3 & 3.3 & 3.3 & 3.8 & 4.4 & 4.8 \\
\hline Current account balance (excl. official transfers, percent of GDP) & -4.1 & -7.9 & -5.3 & -7.2 & -7.5 & -6.4 & -5.0 & -3.7 \\
\hline Current account balance (including official transfers, percent of GDP) & -4.0 & -8.0 & -5.3 & -7.3 & -7.5 & -6.5 & -5.0 & -3.8 \\
\hline Import volume grow th, goods, and services (percent) & -0.3 & 13.6 & 8.7 & 5.5 & 5.8 & 6.6 & 7.2 & 7.3 \\
\hline Import value grow th, goods, and services (percent) & 20.1 & 9.5 & 2.9 & 12.5 & 9.1 & 12.0 & 7.8 & 8.9 \\
\hline Export volume grow th, goods, and services (percent) & 8.8 & -1.5 & 5.9 & 11.0 & 10.5 & 9.7 & 10.5 & 10.5 \\
\hline Export value grow th, goods, and services (percent) & 16.1 & 0.5 & 12.8 & 8.8 & 3.6 & 15.5 & 8.3 & 10.6 \\
\hline Change in the terms of trade (goods and services, percent) $3 /$ & -12.0 & 6.5 & 12.1 & -7.3 & -7.4 & 0.2 & -2.7 & -1.4 \\
\hline
\end{tabular}

Sources: Kenyan authoritıes; and IMr statt estımates and projectıons.

$1 /$ The foreign travel credit is comprised of tw o components, recorded tourism inflow s and an estimate of additional under-reported tourism receipts. 2/ Historical figures include errors and omissions.

3/ Base year: 2000. 
Table 5. Kenya. Financial soundness Indicators of the Banking Sector

(In percent)

\begin{tabular}{|c|c|c|c|c|c|c|}
\hline & Dec-07 & Dec-08 & Jun-09 & Dec-09 & Jun-10 & Sep-10 \\
\hline \multicolumn{7}{|l|}{ Capital adequacy } \\
\hline Regulatory capital to risk-weighted assets & 18.0 & 18.9 & 19.8 & 19.5 & 19.6 & 20.6 \\
\hline Regulatory tier 1 capital to risk-weighted assets & 16.8 & 16.9 & 17.6 & 17.2 & 17.5 & 18.6 \\
\hline Total capital to total assets & 12.0 & 12.6 & 13.0 & 12.7 & 14.0 & 12.8 \\
\hline \multicolumn{7}{|l|}{ Asset quality } \\
\hline Nonperforming loans to total gross loans & 10.6 & 9.0 & 9.0 & 7.9 & 7.4 & 7.0 \\
\hline Nonperforming loans net of provisions to capital & 12.8 & 11.3 & 12.4 & 12.6 & 10.0 & 8.0 \\
\hline Earning assets to total assets & 79.4 & 88.6 & 88.1 & 85.0 & 85.1 & 88.7 \\
\hline \multicolumn{7}{|l|}{ Earning and profitability } \\
\hline Return on assets & 3.0 & 2.8 & 3.1 & 2.9 & 3.5 & 3.4 \\
\hline Return on equity & 27.5 & 25.2 & 28.5 & 24.8 & 31.4 & 31.3 \\
\hline Interest margin to gross income & 34.6 & 34.9 & 35.6 & 35.6 & 33.2 & 33.7 \\
\hline Noninterest expenses to gross income & 50.6 & 51.7 & 49.1 & 50.8 & 47.1 & 49.2 \\
\hline \multicolumn{7}{|l|}{ Liquidity } \\
\hline Liquid assets to total assets & 37.5 & 36.4 & 35.0 & 35.9 & 39.7 & 39.9 \\
\hline Liquid assets to short-term liabilities & 40.0 & 37.0 & 40.6 & 39.8 & 45.1 & 46.7 \\
\hline Liquid assets to total deposits & 47.4 & 46.4 & 45.7 & 46.3 & 50.4 & 51.5 \\
\hline Total loans to total deposits & 71.3 & 73.3 & 75.7 & 72.4 & 68.0 & 69.2 \\
\hline \multicolumn{7}{|l|}{ Sensitivity to market risk } \\
\hline Net open position in foreign exchange to capital & $\ldots$ & 5.6 & 6.1 & 4.9 & 4.9 & 4.8 \\
\hline Interest bearing assets to interest bearing liabilities & 100.5 & 113.0 & 114.4 & 113.2 & 111.6 & 114.4 \\
\hline FX currency denominated assets to total assets & 8.7 & 9.7 & 9.9 & 8.1 & 10.1 & 9.7 \\
\hline FX currency denominated liabilities to total liabilities & 16.3 & 20.2 & 17.6 & 18.1 & 16.3 & 17.1 \\
\hline Spread between lending and deposit rate & 7.5 & 7.8 & 8.8 & 8.7 & 9.4 & 9.3 \\
\hline Total expenses to gross income & 68.9 & 72.0 & 70.0 & 71.4 & 65.6 & 66.1 \\
\hline
\end{tabular}

Source: Central Bank of Kenya. 


\section{Table 6. Proposed Timing of Disbursements and Reviews under the 2011-14 ECF Arrangement}

\begin{tabular}{|c|c|c|c|}
\hline \multirow[b]{2}{*}{ Date of Availability } & \multirow[b]{2}{*}{ Conditions for Fund Disbursement } & \multicolumn{2}{|c|}{ Disbursements } \\
\hline & & Millions of SDRs & Percent of quota 1/ \\
\hline January 31, 2011 & Board approval of the arrangement & 65.136 & 24.00 \\
\hline July 15, 2011 & $\begin{array}{l}\text { Observance of the end-March } 2011 \text { performance criteria } \\
\text { and structural benchmarks, and completion of first review }\end{array}$ & 43.424 & 16.00 \\
\hline October 15, 2011 & $\begin{array}{l}\text { Observance of the end-June } 2011 \text { performance criteria } \\
\text { and structural benchmarks, and completion of second review }\end{array}$ & 43.424 & 16.00 \\
\hline April 15, 2012 & $\begin{array}{l}\text { Observance of the end-December } 2011 \text { performance criteria } \\
\text { and structural benchmarks, and completion of third review }\end{array}$ & 43.424 & 16.00 \\
\hline October 15, 2012 & $\begin{array}{l}\text { Observance of the end-June } 2012 \text { performance criteria } \\
\text { and structural benchmarks, and completion of fourth review }\end{array}$ & 43.424 & 16.00 \\
\hline April 15, 2013 & $\begin{array}{l}\text { Observance of the end-December } 2012 \text { performance criteria } \\
\text { and structural benchmarks, and completion of fifth review }\end{array}$ & 43.424 & 16.00 \\
\hline \multirow[t]{2}{*}{ October 15, 2013} & $\begin{array}{l}\text { Observance of the end-June } 2013 \text { performance criteria } \\
\text { and structural benchmark, and completion of the sixth review }\end{array}$ & 43.424 & 16.00 \\
\hline & & $\underline{\underline{325.680}}$ & $\underline{\underline{120.00}}$ \\
\hline
\end{tabular}

1/ Kenya's quota is SDR 271.4 million. 
Table 7. Kenya: Indicators of Capacity to Repay the Fund, 2010-19

\begin{tabular}{|c|c|c|c|c|c|c|c|c|c|c|}
\hline & \multirow[b]{2}{*}{2010} & \multicolumn{9}{|c|}{ Projections } \\
\hline & & 2011 & 2012 & 2013 & 2014 & 2015 & 2016 & 2017 & 2018 & 2019 \\
\hline \multicolumn{11}{|l|}{$\begin{array}{l}\text { Fund obligations based on existing credit } 1 / \\
\text { (In millions of SDRs) }\end{array}$} \\
\hline Principal & 2.5 & 15.0 & 18.8 & 30.0 & 38.6 & 47.1 & 42.1 & 38.4 & 27.1 & 13.6 \\
\hline Charges and interest & 0.0 & 0.2 & 0.8 & 0.7 & 0.7 & 0.6 & 0.5 & 0.4 & 0.3 & 0.2 \\
\hline \multicolumn{11}{|l|}{$\begin{array}{l}\text { Obligations to the Fund from existing } \\
\text { and prospective credit } 1 / 2 /\end{array}$} \\
\hline (In millions of SDRs) & 2.5 & 15.2 & 20.0 & 31.4 & 40.1 & 48.5 & 49.9 & 74.2 & 80.2 & 79.4 \\
\hline Principal & 2.5 & 15.0 & 18.8 & 30.0 & 38.6 & 47.1 & 48.7 & 73.1 & 79.3 & 78.7 \\
\hline ESF Rapid Access Component (RAC) & 0.0 & 0.0 & 0.0 & 0.0 & 13.6 & 27.1 & 27.1 & 27.1 & 27.1 & 13.6 \\
\hline ECF Arrangements & 2.5 & 15.0 & 18.8 & 30.0 & 25.0 & 20.0 & 21.5 & 46.0 & 52.1 & 65.1 \\
\hline Charges and interest & 0.0 & 0.2 & 1.3 & 1.4 & 1.5 & 1.4 & 1.3 & 1.1 & 0.9 & 0.7 \\
\hline \multicolumn{11}{|l|}{$\begin{array}{l}\text { Obligations to the Fund from existing and } \\
\text { prospective credit } 1 / 2 /\end{array}$} \\
\hline In millions of U.S. dollars & 4.0 & 24.2 & 31.9 & 50.1 & 63.8 & 77.2 & 79.5 & 118.2 & 127.6 & 126.4 \\
\hline In percent of gross international reserves & 0.1 & 0.5 & 0.6 & 0.8 & 0.8 & 0.8 & 0.8 & 1.0 & 1.0 & 0.9 \\
\hline In percent of exports of goods and services & 0.0 & 0.3 & 0.3 & 0.4 & 0.5 & 0.5 & 0.5 & 0.7 & 0.7 & 0.6 \\
\hline In percent of debt service & 1.1 & 5.9 & 6.9 & 10.4 & 11.7 & 13.4 & 11.0 & 13.6 & 12.6 & 11.4 \\
\hline In percent of GDP & 0.0 & 0.1 & 0.1 & 0.1 & 0.1 & 0.1 & 0.1 & 0.2 & 0.2 & 0.1 \\
\hline In percent of quota & 0.9 & 5.6 & 7.4 & 11.6 & 14.8 & 17.9 & 18.4 & 27.4 & 29.5 & 29.3 \\
\hline \multicolumn{11}{|l|}{ Outstanding Fund credit (end-of-period) 1/ 2/ } \\
\hline In millions of SDRs & 273.2 & 410.2 & 478.3 & 535.1 & 496.6 & 449.4 & 400.8 & 327.6 & 248.4 & 169.7 \\
\hline In millions of U.S. dollars & 435.4 & 653.1 & 762.0 & 852.7 & 790.6 & 715.5 & 638.1 & 521.6 & 395.5 & 270.2 \\
\hline In percent of gross international reserves & 10.7 & 14.6 & 14.6 & 12.9 & 10.1 & 7.7 & 6.1 & 4.6 & 3.2 & 2.0 \\
\hline In percent of exports of goods and services & 4.9 & 7.0 & 6.9 & 7.0 & 6.3 & 5.0 & 4.0 & 3.2 & 2.1 & 1.3 \\
\hline In percent of GDP & 1.4 & 1.8 & 1.9 & 1.9 & 1.6 & 1.3 & 1.0 & 0.7 & 0.5 & 0.3 \\
\hline In percent of quota & 100.7 & 151.1 & 176.2 & 197.2 & 183.0 & 165.6 & 147.7 & 120.7 & 91.5 & 62.5 \\
\hline \multicolumn{11}{|l|}{ Memorandum items: } \\
\hline Nominal GDP & 32,088 & 35,611 & 40,349 & 45,172 & 50,632 & 56,726 & 63,789 & 71,463 & 79,600 & 88,641 \\
\hline Exports of goods and services & 8,845 & 9,340 & 10,977 & 12,098 & 12,644 & 14,371 & 15,915 & 16,520 & 18,409 & 20,538 \\
\hline Gross international reserves & 4,083 & 4,488 & 5,215 & 6,609 & 7,807 & 9,294 & 10,461 & 11,412 & 12,450 & 13,742 \\
\hline Debt service (in millions of U.S. dollars) $3 /$ & 366 & 413 & 461 & 483 & 543 & 578 & 722 & 868 & 1,009 & 1,112 \\
\hline Quota (in millions of SDRs) & 271.4 & 271.4 & 271.4 & 271.4 & 271.4 & 271.4 & 271.4 & 271.4 & 271.4 & 271.4 \\
\hline
\end{tabular}

Sources: Fund staff estimates; and projections.

1/ For 2010, outstanding credit and Fund obligations are as of end-November 2010.

2/ Assumes access of 120 percent of quota during 2011-13 and semi-annual disbursements starting in July 2011. Outstanding credit at end-2010 stands at SDR 270.7 million, equivalent to 99.7 percent of quota.

3 / Total debt service includes repayments to the IMF. 


\section{Appendix I. Letter of Intent}

January 17,2011

Mr. Dominique Strauss-Kahn

Managing Director

International Monetary Fund

$70019^{\text {th }}$ Street NW

Washington, DC 20431 USA

Dear Mr. Strauss-Kahn:

Following shocks associated with the post-election violence of 2008, consecutive droughts, and the impact of the global economic downturn in 2009, Kenya's economy is recovering steadily. Economic prospects have been boosted by the promulgation of our new constitution on August 27, which will both help to address the long-standing social and political problems that have held back our growth potential and stimulate renewed interest from foreign investors.

For 2010 and beyond, the main goal of our policies is to bring Kenya back to the growth trajectory set in the medium-term plan of Vision 2030. Key priorities include investing in infrastructure and the energy sector to deal with the challenges of climate change, spending on implementation of the new constitution, and ensuring adequate allocations to the social sectors. At the same time, we intend to strike a balance between devoting resources for these priorities and gradual fiscal adjustment to ensure long-term debt sustainability. Monetary policy will aim at meeting the medium-term inflation target, maintaining the floating exchange rate regime, and gradually building up foreign exchange reserves to meet our import coverage targets. Structural reforms for the near and medium-term will focus on strengthening the public financial management framework, modernizing and simplifying the tax system, increasing access to financial services while ensuring adequate supervision, and enhancing the business environment.

The main features of the government's economic reform program are described in the attached Memorandum of Economic and Financial Policies (MEFP), which lays out the economic objectives and policies of the Government of Kenya for the 2010/11-2013/14 period. These objectives and policies are consistent with the aspirations of Kenya's Vision 2030. Based on our balance of payments needs and the policies described in the MEFP, we request that the Executive Board approve a three-year Fund arrangement under the Extended Credit Facility (ECF) in the amount of SDR 325.68 million in total for the period January 2011-January 2014, and the first disbursement of SDR 65.14 million.

The government believes that the policies set out in this letter are adequate to achieve the objectives of its program, but it will take any further measures that may become appropriate 
for this purpose. The government will consult with the Fund on the adoption of these measures, and in advance of revisions to the policies contained in the MEFP, in accordance with the Fund's policies on such consultations. The Government of Kenya will provide such information as the Fund may request in connection with progress in implementing its economic and financial policies.

We have no objection to publication of the staff report for the request of the ECF arrangement, this letter of intent, and the attached memorandum of economic and financial policies and technical memorandum of understanding.

Sincerely yours,

$$
/ \mathrm{s} /
$$

Uhuru M. Kenyatta

Deputy Prime Minister and Minister for Finance
Njuguna Ndung'u Governor Central Bank of Kenya

Attachments: Memorandum on Economic and Financial Policies Technical Memorandum of Understanding 


\section{Attachment 1. Memorandum of Economic and Financial Policies for 2011-13 under the Three-Year Program of the Extended Credit Facility Arrangement}

\section{BACKGROUND}

1. Kenya stands at a critical juncture in its post-independence history. The new constitution approved in August 2010 has raised expectations in the country that a permanent solution to long-standing social and political challenges may be achievable. The new legal framework envisaged in the constitution will allow for strong institutions to provide checks and balances on the government, judiciary reform, the devolution of resources to the regions, land-ownership reform, and improvements in the public expenditure framework. The successful implementation of the new constitution will enhance the chances of achieving Kenya's Vision 2030 aspirations of substantial improvements in the economic, social and political fronts.

2. The economic gains achieved since 2003 were disrupted in $2008 / 09$, but recovery is underway. Our economy staged a strong recovery during 2003-07, in response to bold economic and structural reforms. Real GDP growth reached 7 percent by 2007 before suffering major setbacks as a result of the post-election violence in early 2008, a severe drought, high international commodity prices, and spillover effects of the global financial crisis. These shocks threatened to reverse the gains we had achieved in terms of expanding employment opportunities for our people and fighting poverty. A concerted effort by the government, including easing of macroeconomic policies, has begun to bear fruit with recovery now underway. However, our per capita GDP has not increased sufficiently to make a dent on poverty and reduce unemployment, and long-standing energy and infrastructure gaps remain. Despite improved macroeconomic policy management, strong growth needs bolder measures to address governance-related weaknesses, deep-seated political differences and an unfavorable business environment.

3. The government is committed to achieve a higher and sustainable growth of the economy in a more equitable environment, with increased employment opportunities. Our first Medium-Term Plan (MTP) of Vision 2030 (Poverty Reduction Strategy PaperPRSP) aims at raising real GDP growth to 10 percent. Achieving this growth will enable us to direct more resources to pro-poor sectors including education and health to achieve social development and reduce poverty. With agriculture the mainstay of the economy, we plan to improve productivity in agriculture by reducing the cost of farm inputs, improving farm prices, expanding irrigation to new areas, increasing water access, and preserving the environment. Public investment in construction and upgrading of the road, railroad network, ports, and information and communication technology will be complemented by private sector investment under the Public Private Partnership (PPP) framework. In addition, to meet the power needs for the expanding economy, the National Electricity Supply Master Plan will identify new generation and supply sources with the goal of tripling the national electricity 
supply of dependable energy to 3,000 megawatts by 2018, with emphasis on the development of alternative power sources, especially geothermal power.

\section{RECENT ECONOMIC DEVELOPMENTS}

\section{Recent data indicate that Kenya's economy is bouncing back from the major}

shocks in 2008/2009. Real GDP growth reached 5 percent in the first half of 2010 reflecting in part the lagged effects of last year's fiscal and monetary stimulus, as well as favorable weather conditions that supported a rebound in agriculture growth. The government's renewed efforts to upgrade infrastructure together with lower interest rates supported a strong expansion in construction activity. By June 2010, credit growth had picked up, with an increasing share going to personal loans helped by rapid modernization of financial services that has seen increased financial inclusion. Inflation has remained well below the 5 percent target, helped by ample food supply and low telecommunication rates driven down by intense competition in a vibrant mobile telephony market.

5. Low reserves and increasing public debt entail vulnerabilities. Despite Fund support under the ESF and the 2009 SDR allocation, international reserves have remained below target as capital inflows remain subdued relative to other developing economies. Increased tea exports, tourism receipts and remittances helped improve the external current account, but the risk of adverse exogenous shocks remains high. Notwithstanding strong public investment that will support future growth, public debt in net present value (NPV) terms now lies at the 40 percent debt-to-GDP (or over 45 percent of GDP in nominal terms) ceiling set in our medium-term debt-management framework, which is consistent with the debt sustainability threshold for a country like Kenya - a medium performer according to the World Bank 's Country Policy and Institutional Assessment (CPIA) Index. Our debt strategy aims at maintaining a sustainable level of debt, diversifying sources of financing with emphasis on concessional external financing and shifting the composition of domestic debt towards long term maturities to minimize both cost and risk. However, pressing needs for large investment in infrastructure - especially in the energy sector - and the cost of implementing the constitution, will keep the fiscal deficit at around $7 \frac{1}{2}$ percent of GDP for a second consecutive year in 2010/11. While recognizing these increased demands, we will ensure that our public debt including contingent liabilities remains within sustainable path over the medium term.

\section{Program ObJectives and Macroeconomic Policies}

\section{The 2011-13 Economic Program under the three-year ECF will help to protect} Kenya's external position, while preserving stability and supporting the conditions for sustainable growth. The program will aim at addressing balance-of-payments financing needs arising from the impact on domestic demand of the government's investment plan, especially into geothermal energy; providing a reserve cushion to help address adverse 
external shocks; and maintaining macroeconomic stability in the implementation of the decentralization process envisaged in the new constitution.

7. Our policies outlined in the 2010 Medium-Term Budget Strategy Paper and updated in the 2011 Budget Outlook Paper aim at consolidating the economic recovery and put our economy back onto a high and sustainable growth path. Over the program period, we expect real GDP growth to rise close to 7 percent, with an increased reliance on domestic private savings and investment consistent with a decline in the external current account deficit to about 5 percent of GDP. To this end, we will up-scale investment in the key priority social and economic sectors as well as in critical physical infrastructure such as roads, rail, power generation, and ports. This will be complemented by deepening structural reforms in the area of governance, public financial management; public services, business regulation, and the financial sector, with support from the World Bank and other development partners. These efforts will help reduce the cost of doing business and thus enhance Kenya's competitiveness as well as private sector investment and growth.

\section{A. Fiscal Policy}

8. Policy objective: Fiscal policy will continue to support economic activity while allowing for implementation of the new constitution within a context of sustainable public financing. As such, we are committed to gradually reduce the overall fiscal deficit to about 5 percent of GDP over the medium term. This will help to bring down the debt-to-GDP ratio to a sustainable level and contribute to reducing the external imbalance. Fiscal measures will seek a reduction of the primary budget deficit to about 2 percent of GDP in 2012/13 (performance criterion) from 3.8 percent projected for 2010/11. The government will maintain the fiscal revenue-to-GDP ratio at around 25 percent and keep budgetary expenditures consistent with medium-term priorities while providing sufficient room for implementation of the new constitution.

9. Implementation of the new constitution: We plan to provide sufficient resources to implement the new constitution to meet the timeline provided in the transitional arrangement. Constitution-related expenditures include drafting of new laws; review of existing legislation to conform with the provisions of the new constitution; stakeholder participation and civic education; winding up and setting up new offices; capacity building for counties; construction and/or rehabilitation of county assemblies, National Assembly and Senate; and, establishment of the new judicial system. While final costing is not yet completed at this stage, we have set aside Ksh 4 billion in this current fiscal year and Ksh 9.5 billion for the next year for this purpose. As detailed costing of these expenditures will become clear in the course of the program, we plan to update the fiscal targets to ensure smooth implementation of this national objective, in the context of program reviews. Updating of the targets will take into account available support from development partners while ensuring continued public finance sustainability. 
10. Revenue measures: We plan to appoint a Tax Reform Commission (TRC) that will be in charge of simplifying our tax code in line with best practices, in order to help improve tax compliance, minimize delays, and raise revenue. Key measures we plan to implement include rationalizing existing tax incentives, expanding the income tax base and removing tax exemptions as envisaged in the constitution. In addition, we will improve the infrastructure for enhanced collection of property taxes to strengthen the revenue base of the new counties within the devolved system. We have requested technical assistance from the Fund in support of these reform efforts.

11. We will modernize our VAT legislation to help improve administration and compliance (structural benchmark). VAT reform will include accompanying policies to reinforce its effectiveness, with measures to rationalize VAT expenditures and exemptions as well as measures to improve efficiency of VAT filing, VAT refund processing, and VAT withholding. We will continue to rely on assistance from the World Bank and the Fund in these areas.

12. Revenue administration measures: We will continue with efforts to increase automation of KRA processes to help combat tax evasion and to support customs reform. This will include more intensive use of cameras and digital video clips to accompany verification reports and making further progress in rolling out the Electronic Cargo Tracking System. To further enhance governance, we will continue our policies to reduce face-to-face interaction with KRA officials and to shift from paper to electronic based applications.

13. Expenditure measures: We intend to maintain a policy of rationalizing recurrent expenditure including restraining growth in wage payments to free resources towards development expenditure. Over the medium term, we will continue to protect pro-poor wage expenditure in health and education sectors while limiting new recruitment in the public service to sustainable levels. Given the need to implement the new constitution, we will ensure that adequate resources are set aside for this purpose. We will also ensure that development expenditure remains supportive of critical infrastructure that will help reduce the cost of doing business and crowd in private sector investment in areas identified in the Vision 2030's first MTP.

14. Expenditure control will be enhanced with the implementation of a new public finance management (PFM) legislation. In line with constitutional implementation process, a draft PFM Bill will be submitted to the Commission for the Implementation of the Constitution by December 2011 (structural benchmark) for consideration and submission to parliament. The new PFM legislation will help to accelerate reforms in public financial management under a coordinated strategy to revitalize Public Finance Management Reforms, as well as facilitate adoption of a Single Treasury Account by June 2012 to strengthen cash management system and improve resource management (structural benchmark). To enhance effectiveness of the Public Procurement Oversight Authority, we will review the procurement legislation and enhance its capacity. In addition, we will implement the Integrated Financial 
Management Information System (IFMIS) across government Ministries and Departments. The IFMIS will ensure effective budgetary management at all stages, including budget preparation, treasury management, budgetary control, fiscal reporting and preparation of annual accounts. The IFMIS will be eventually rolled out to the county governments. Meanwhile, we will deepen implementation of Performance Based Budgeting that will help improve control and accountability.

15. Poverty and social impact: We will continue pursuing the poverty reduction objectives and MDGs. Public investment is still needed to achieve the MDGs, but because fiscal space remains limited, strong donor assistance will be needed to raise and sustain high public investment levels. Nevertheless, we will continue to protect priority social expenditures to ensure progress in achieving social objectives as elaborated in the social pillar of our Vision 2030 MTP (indicative target). We will also ensure efficient utilization of existing resource allocations to these critical social sectors while we undertake additional measures to boost benefits to mitigate the poverty impact of food insecurity problems to vulnerable members of our society. Over the medium term, we plan to rationalize and expand existing social protection programs, while at the same time ensure adequate spending on education and health.

16. Financing policy: With gradual fiscal consolidation, we expect the share of domestic financing to decline from 70 percent of financing the current fiscal year to about 60 percent next year. The composition for subsequent years will depend on the timing of the issuance of a sovereign bond. External financing will come mainly from concessional external sources, including about US\$2 billion in project loans in the next two fiscal years, mainly from the World Bank (WB) and the African Development Bank (AfDB). The WB is expected to provide budget support of US $\$ 100$ million through a Development Policy Loan In the absence of concessional financing, the government will maintain nonconcessional financing including guarantees at below US\$700 million in the remainder of fiscal year 2010/11 and US\$700 million in the year 2011/12 (performance criterion). The nonconcessional financing including the sovereign bond proceeds will be limited to investment projects that demonstrate revenue streams and of high social returns. While we plan to issue a sovereign bond in 2012/13, we will monitor developments in the international capital markets with a view to issue the bond early to take advantage of the favorable interest rates, market conditions permitting. As before, we will continue with our policy of not incurring external arrears (performance criterion).

17. Contingent liabilities management: Following issuance of the government's Public Procurement and Disposal regulations in March 2009, the government is committed to adopt a PPP policy that seeks to address any gaps in the legal and regulatory framework that could impede successful implementation of the PPP agenda, and provide for a clear and mandatory regulatory framework to guide PPP investments. As the government embraces the PPP framework, we will introduce administrative arrangements and processes that help guide the selection and implementation of projects. We will scrutinize PPP projects carefully to 
preserve fiscal discipline and safeguard the interest of tax payers. All guarantees provided under the PPP agenda, together with all other contingent liabilities will be integrated into the debt management process.

\section{B. Monetary Policy}

18. Policy objective: The CBK will keep the 5 percent inflation target and maintain a floating exchange rate regime. For that purpose, the CBK will pursue a prudent money supply growth and embark on a gradual accumulation of international reserves that will lift the import coverage to over four months of following year's imports of goods and services by the end of the program period (performance criterion), consistent with the CBK statutory obligation. Monetary policy will focus on its medium-term inflation target, with net domestic assets as an intermediate target (performance criterion). To that effect, the CBK will monitor the appropriate monetary aggregates to ensure consistency with market developments to pre-empt any inflationary pressures, and enhance coordination of monetary and fiscal policy to minimize the liquidity impact of government financial transactions as well as respond timely to external shocks.

19. Monetary policy framework: The government will set up a strategy to move away from monetary targeting towards an appropriate monetary policy framework that will take into account the expected continuation of a reduction of velocity of circulation and the rise in the money multiplier explained by deeper financial intermediation and increased modernization of the financial system. With inflation targeting a viable option, the CBK will continue to refine the required infrastructure for its successful adoption. As a step towards this goal and with a view to strengthening central bank accountability in the conduct of monetary policy, the Minister of Finance will continue to convey to the CBK the inflation objective for the fiscal year after the budget presentation to parliament. The CBK will report on deviations of 2 percent or more from the stated inflation target. In line with the new constitution, the CBK Act will be refined to enhance its operational mandate within an improved framework of transparency and accountability, while preserving its operational autonomy.

\section{Financial Policy}

20. Policy objective: We will step up the efforts to strengthen the financial infrastructure to adapt to the new challenges associated with the modernization of the banking system, including cross-border operations and mobile banking. Measures conducive to that goal are the following:

- $\quad$ Upgrade the crisis management frameworks to deal with the challenges of crossborder banking activities, including implementation of consolidated supervision.

- $\quad$ Reinforce prompt corrective action by the banking supervision authority, by amending the Banking Act by March 2011 (structural benchmark). 
- $\quad$ Give powers to the Deposit Protection Fund Board to hold and sell unrealizable securities after winding up an institution.

- $\quad$ Strengthen risk-based supervision including adopting a time-bound program to put in place an assessment of conglomeration risk of relevant local groups.

- $\quad$ Expand supervisory procedures to ensure full monitoring of financial business driven by bank agents under the newly introduced bank agency model and through the use of mobile banking.

- $\quad$ Modify the CBK Act to allow the central bank to share or disclose information obtained from surveillance of authorized foreign exchange bureaus for criminal investigations.

- Introduce legislation by March 2011 to allow the demutualization of the Nairobi Stock Exchange, in an effort to strengthen capital markets (structural benchmark).

- $\quad$ Enforce compliance of Retirement Benefit Authority (RBA) rules on National Social Security Fund (NSSF), in particular the appointment of external fund managers and external custodians.

- $\quad$ Fully implement the Crime and Anti-Money Laundering Act of 2009, including introducing necessary by-laws and regulations.

21. Financial access: Financial access to the population has improved, with a share of 40 percent of people with access to formal financial services in 2009, up from 26 percent in 2006. Innovative financial products and services are expanding financial access, helped by the use of mobile-based technology and widespread use of mobile phones (one cell phone per adult). According to World Bank estimates, about $3 / 4$ of the population already transfer cash equivalent to 20 percent of GDP annually, and the number of mobile-based deposit accounts products is growing rapidly. The recent introduction of a Credit Reference Bureau and the agency banking model are helping reaching previously unbanked segments of the population by mitigating credit risk and reducing transaction costs. Following approval of the Microfinance Act in 2008, the CBK has licensed three deposit-taking microfinance institutions (MFIs), and many other institutions have started the licensing process. We are considering the following measures to support the drive towards higher credit access:

- $\quad$ Facilitate the increase in information coverage by the newly introduced Credit Reference Bureau.

- Amend the Microfinance Act to facilitate the use of third party agents by DepositTaking Microfinance Institutions. 
- $\quad$ Complete the regulatory and supervisory frameworks for Savings and Credit Cooperatives (SACCOs) to help improve consumers' confidence.

- Implement currency centers out of Nairobi to reduce cash-in-transit costs to banks' branches.

22. More fundamental reforms are needed to ensure adequate access to productive credit to small and medium-sized enterprises. Some elements of the constitutional reform would favor broadening access to credit, such as the envisaged decentralization and judiciary reform. Recently introduced regulation and supervision of specialized institutions such as MFIs and SACCOs are necessary steps to help strengthen their financial soundness. These institutions have potentially large financial outreach that could favor lower transaction costs in lending to micro-enterprises, in particular in the rural area where about 250 deposit-taking SACCOs already constitute an important source of credit for rural households. Many of these institutions experience the same difficulties as other financial institutions in trying to expand lending to the productive sector, namely a lengthy and costly collateralization, complex and uncertain loan recovery processes in the event of default, inefficient registry, and weak legal basis for the use of alternative risk mitigators. In adapting Kenya's institutional framework to the new constitution, reform in the following areas will be assessed:

- Unify the framework for the use of immovable property as collateral, including land, and eliminating restrictions to free contracting. Create a streamlined and transparent system for the treatment of noncorporate movable assets as collateral.

- $\quad$ Significantly simplify the regime for stamp duties charged at different stages of collateralization and execution of collateral.

- $\quad$ Create a unique registry for encumbrances within the Register General, and establish a simplified registration process. In amending the Company's Act, remove limits to the type of security interests that a company can register, and give the same preference to creditors charging fixed and floating rates (the latter normally preferred for lending to some agriculture activities).

- $\quad$ Reform the legal framework and tax treatment of alternative risk mitigators such as hire purchases, invoice discounting and factoring, and warehouse receipts. Over time, local governments may promote the use of these alternative risk mitigators by financially sound SACCOs.

\section{SAFEguARdS AND Statistics IsSUES}

23. The government remains committed to protecting the financial soundness of the $\mathrm{CBK}$, and to adhering to the principles of good governance and best practices, including those encapsulated in the IMF's safeguards policy. A safeguards assessment of the CBK was conducted in September 2009, which found that the CBK had made notable progress in 
strengthening its safeguards. The bank is committed to implementing measures to address the remaining vulnerabilities, further strengthen the CBK's operations, and minimize the possibility of misreporting and misuse of Fund resources.

24. On statistical issues, the government plans to release a revised set of national accounts data in 2012. The government is also taking steps to improve the monitoring of fiscal data, in particular, the preparation, on a regular basis, of summary statistics on domestic arrears and pending bills. Moreover, the government intends to develop a framework for systematically monitoring productivity changes in the economy, and to produce, on a regular basis, trade data. Kenya participates in the General Data Dissemination System (GDDS) project for Anglophone Africa.

\section{Program Monitoring For 2011/12}

25. Technical memorandum of understanding (TMU). The program will be monitored using the definitions, data sources, and frequency of monitoring set out in the accompanying TMU. The government will make available to Fund staff all data appropriately reconciled and on a timely basis, as specified in the TMU.

26. Performance criteria and structural benchmarks. Annex 1 shows the quantitative performance criteria to be used in monitoring performance in 2011. Structural benchmarks, with corresponding dates, are identified in Annex 2.

27. Program review. The first review under the ECF arrangement, assessing end-March 2011 performance criteria, will be completed by July 15 2011. This review will focus on maintaining fiscal discipline while keeping expenditure in favor of social and economic spending as a priority. The second review under the ECF arrangement, assessing end-June performance criteria, is expected to be completed by October 15, 2011. The third review under the ECF arrangement assessing end-December performance criteria, is expected to be completed by April 15, 2012.

28. Coverage. Fiscal performance criteria will cover the central government, but the authorities' will prepare to expand coverage to include the general government as early as possible, in anticipation of fiscal decentralization. 


\section{Annex 1. Kenya: Proposed Performance Criteria}

(In billions of Kenyan shillings; unless otherwise indicated)

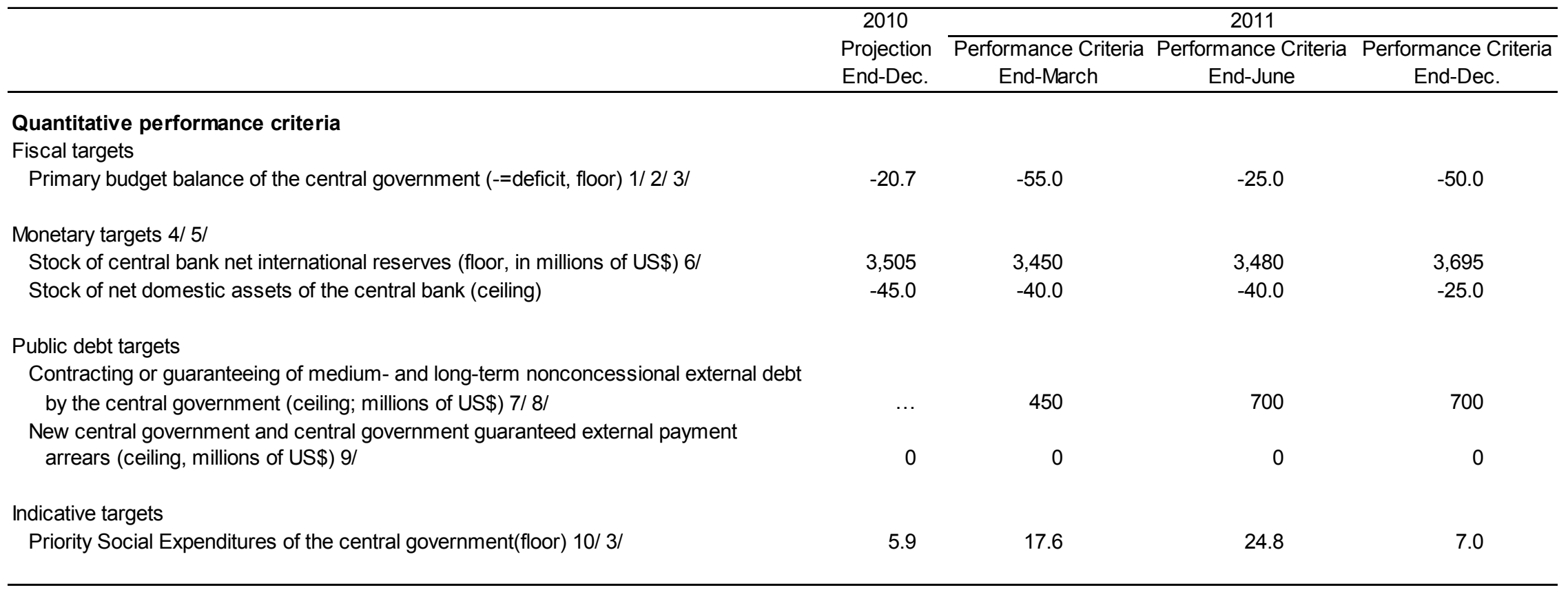

1/ The primary budget balance of the central government is defined as overall balance including grants, minus concessional project loans, plus interest payments. 2/ For 2010, end-March 2011, and end-June 2011: cumulative flow, from October 1, 2010 (beginning of the second quarter of the 2010/2011 fiscal year).

3/ For end-December 2011: cumulative flow, from July 1, 2011 (beginning of the 2011/2012 fiscal year).

4/ For program monitoring, the daily average for the month when testing dates are due.

5/ The NIR floor will be adjusted upward (downward) by the excess (shortfall) of external budgetary support (grants and loans) and external commercial debt relative to the programmed amounts. The NDA ceiling will be adjusted downward (upward) by the excess (shortfall) of external budgetary support (grants and loans) and external commercial debt relative to the programmed amounts.

6/ Excludes encumbered reserves.

7/ Cumulative flow of contracted debt, from January 1, 2011.

8/ For 2011, the targets do not include the planned issue of sovereign bond that is now scheduled for 2012/13 fiscal year.

9/ Continuous.

10/ For 2010, end March 2011, and end-June 2011: the target is cumulative from July 1, 2010. 


\section{Annex 2. Kenya. Structural Benchmarks under the Extended Credit Facility Arrangement}

\begin{tabular}{|c|c|}
\hline \multirow{4}{*}{$\begin{array}{l}\text { Measure } \\
\text { Tax measures } \\
\text { Submit VAT legislation to help improve administration and compliance. } \\
\text { Macro criticality: The VAT reform will allow for higher mobilization of revenue that } \\
\text { will reduce the fiscal imbalance. }\end{array}$} & Proposed Time Frame \\
\hline & \multirow{3}{*}{ Second Review } \\
\hline & \\
\hline & \\
\hline \multicolumn{2}{|l|}{ Expenditure control } \\
\hline Submit PFM legislation to the Commission for the Implementation of the & Third Review \\
\hline Constitution, to help accelerate reforms in public financial management. & \\
\hline \multirow{2}{*}{\multicolumn{2}{|c|}{$\begin{array}{l}\text { Macro criticality: PFM management legislation is crucial for increasing both } \\
\text { spending efficiency and improving fiscal management. }\end{array}$}} \\
\hline & \\
\hline $\begin{array}{l}\text { Adopt a Single Treasury Account to strengthen cash management and improve } \\
\text { resource management. }\end{array}$ & Fourth Review \\
\hline \multirow{2}{*}{\multicolumn{2}{|c|}{$\begin{array}{l}\text { Macro criticality: Single Treasury Account adoption will improve both liquidity } \\
\text { management and expenditure control. }\end{array}$}} \\
\hline & \\
\hline \multicolumn{2}{|l|}{ Banking supervision } \\
\hline $\begin{array}{l}\text { Amend the Banking Act to reinforce prompt corrective action by the banking } \\
\text { supervision authority. }\end{array}$ & First Review \\
\hline \multirow{2}{*}{\multicolumn{2}{|c|}{$\begin{array}{l}\text { Macro criticality: Reinforcing the banking supervision authority is crucial to } \\
\text { reducing the risk of macroeconomic instability. }\end{array}$}} \\
\hline & \\
\hline \multicolumn{2}{|l|}{ Capital markets } \\
\hline $\begin{array}{l}\text { Introduce legislation to allow the demutualization of the Nairobi Stock Exchange, to } \\
\text { remove the conflict of interest from the governing body of the exchange and to } \\
\text { strengthen capital markets. }\end{array}$ & First Review \\
\hline $\begin{array}{l}\text { Macro criticality: Demutualization of the Nairobi Stock Exchange is essential for the } \\
\text { development of deeper financial markets that will enhance financial stability. }\end{array}$ & \\
\hline
\end{tabular}




\section{Attachment 2. Technical Memorandum of Understanding}

1. This memorandum sets out the understandings between the Kenyan authorities and the IMF regarding the definitions of quantitative performance criteria and indicative targets, their adjusters and data reporting requirements for the three-year Extended Credit Facility (ECF) arrangement.

\section{Quantitative Performance Criteria And Benchmarks}

2. Quantitative performance criteria are proposed for March 31, 2011, June 30, 2011 and December 31, 2011 with respect to:

- $\quad$ the primary balance of the central government including grants, excluding external concessional project loans, cash basis (floor);

- $\quad$ the net official international reserves (NIR) of the Central Bank of Kenya (CBK) (floor);

- $\quad$ the net domestic assets (NDA) of the CBK (ceiling);

- $\quad$ nonconcessional medium- and long-term external debt contracted or guaranteed by the central government (ceiling); and

- $\quad$ medium- and long-term external public debt arrears (continuous ceiling).

3. The program sets indicative targets for March 31, 2011; June 30, 2011; and December 31, 2011 with respect to:

- $\quad$ priority social spending of the central government (floor).

\section{Performance Criterion on the Primary Balance Including Grants of the Central Government}

4. The central government primary balance excluding external concessional project loans on cash basis is defined as central government revenues and grants minus expenditures and net lending, plus due interest payments and investment expenditure financed through external concessional project loans, adjusted for cash basis.

5. For program purposes, the central government primary balance excluding external concessional project loans on cash basis is cumulative from October 1, 2010 and will be measured from the financing side as the sum of the following: (a) the negative of net domestic financing of the central government; (b) the negative of net external financing of the central government, excluding external concessional project loans; and (c) domestic and external interest payments of the central government. For the December 31, 2011 test 
date, the central government primary balance including grants will be measured cumulative from July 1, 2011.

The above items are defined as follows:

- Net domestic financing of the central government is defined as the sum of:

net domestic bank financing;

$>$ net nonbank financing;

$>$ change in the stock of domestic arrears of the central government; and

$>$ proceeds from privatization.

- Net external financing excluding external concessional project loans is defined as the sum of:

$>$ disbursements of external nonconcessional project loans, including securitization;

$>$ disbursements of budget support loans;

$>$ principal repayments on all external loans;

$>$ net proceeds from issuance of external debt;

$>$ any exceptional financing (including rescheduled principal and interest);

$>$ net changes in the stock of short-term external debt; and

$>$ any change in external arrears including interest payments.

- External concessional project loans of the central government are defined as external project loans contracted by the central government, which are considered concessional according to the definition in paragraph 11. All other external project loans are deemed nonconcessional external project loans.

- Domestic and external interest payments of the central government are defined as the due interest charges on domestic and external central government debt.

\section{Performance Criterion on the Net International Reserves of the Central BANK OF KENYA}

6. The net official international reserves (stock) of the CBK will be calculated as the difference between total gross official international reserves and official reserve liabilities.

- Gross official international reserves are defined as the sum of:

$>\quad$ the CBK's holdings of monetary gold (excluding amounts pledged as collateral);

holdings of Special Drawing Rights (SDRs), including the August 28, 2009 General allocation and the September 9, 2009 Special allocation; 
CBK holdings of convertible currencies in cash or in nonresident financial institutions (deposits, securities, or other financial instruments).

- Gross official international reserves exclude:

$>\quad$ the reserve position in the IMF;

pledged, swapped, or any encumbered reserve assets, including but not limited to reserve assets used as collateral or guarantees for third-party external liabilities;

$>\quad$ deposits with Crown agents; and

precious metals other than gold, assets in nonconvertible currencies and illiquid foreign assets.

- Official reserve liabilities are defined as:

$>\quad$ the total outstanding liabilities of the CBK to the IMF except those arising from the August 28, 2009 SDR general allocation and the September 9, 2009 SDR special allocation;

$>\quad$ convertible currency liabilities of the CBK to nonresidents with an original maturity of up to and including one year;

$>\quad$ commitments to sell foreign exchange arising from derivatives (such as futures, forwards, swaps, and options).

- The following adjustors will apply to the target for NIR:

$>\quad$ If budgetary support (external grants and loans) and external commercial debt exceed the programmed amounts, the target for NIR will be adjusted upward by the difference.

If budgetary support (external grants and loans) and external commercial debt fall short of the programmed amounts, the target for NIR will be adjusted downward by the difference.

7. NIR are monitored in U.S. dollars, and, for program monitoring purposes, assets and liabilities in currencies other than the U.S. dollar shall be converted into dollar equivalent values, using the exchange rates as specified in Table 1, and net international reserves will be computed as the daily average for the month when the testing date is due. 


\section{Performance Criterion on the Net Domestic Assets (NDA) OF the Central BANK OF KENYA}

8. Net domestic assets are defined as reserve money minus NIR converted in shillings at the accounting exchange rate of 81 shillings for one U.S. dollar, plus medium and long term liabilities (i.e., liabilities with a maturity of one year or more) of the CBK, including those arising from the August 28, 2009 SDR General allocation and the September 9, 2009 SDR Special allocation; minus the value in shillings of encumbered reserves converted at the accounting exchange rate of 81 shillings for one U.S. dollar.

- $\quad$ NDA is composed of:

$>\quad$ net CBK credit to the central government;

$>\quad$ outstanding net credit to domestic banks by the CBK (including overdrafts); and

$>\quad$ other items net.

Reserve money is defined as the sum of:

$>\quad$ currency in circulation; and
$>\quad$ required and excess reserves.

- $\quad$ The following adjustors will apply to the target for NDA:

$>\quad$ If budgetary support (external grants and loans) and external commercial debt exceed the programmed amounts, the target for NDA will be adjusted downward by the difference.

$>\quad$ If budgetary support (external grants and loans) and external commercial debt fall short of the programmed amounts, the target for NDA will be adjusted upward by the difference.

9. NDA are monitored in shillings, and will be computed as the daily average for the month when the testing date is due.

\section{Performance Criterion on Nonconcessional External Debt Contracted or GuARANTEED BY THE CENTRAL GovernMENT}

10. The definitions of "debt" and "concessional borrowing" for the purposes of this memorandum of understanding are as set out in point 9 of Executive Board Decision No. 6230-(79/140), as subsequently amended, including by Executive Board Decision No. 14416-(09/91), effective December 1, 2009. This definition also includes the following: 
- Debt is understood to mean a direct, that is, not contingent, liability, created under a contractual agreement through the provision of value in the form of assets (including currency) or services, which requires the obligor to make one or more payments in the form of assets (including currency) or services at some future point(s) in time; these payments will discharge the principal and/or interest liabilities incurred under the contract. Debts can take a number of forms, the primary ones being as follows:

$>\quad$ loans, that is, advances of money to the obligor by the lender made on the basis of a undertaking that the obligor will repay the funds in the future (including deposits, bonds, debentures, commercial loans, and buyers' credits) and temporary exchanges of assets that are equivalent to fully collateralized loans under which the obligor is required to repay the funds, and usually pay interest, by repurchasing the collateral from the buyer in the future (such as repurchase agreements and official swap arrangements);

$>\quad$ suppliers' credits, that is, contracts where the supplier permits the obligor to defer payments until sometime after the date on which the goods are delivered or services are provided; and

leases, that is, arrangements under which property is provided which the lessee has the right to use for one or more specified period(s) of time that are usually shorter than the total expected service life of the property, while the lessor retains title to the property. For the purpose of this guideline, the debt is the present value (at the inception of the lease) of all lease payments expected to be made during the period of the agreement excluding those payments that cover the operation, repair or maintenance of the property.

- Under the definition of debt set out above, arrears, penalties, and judicially awarded damages arising from failure to make payment under a contractual obligation that constitutes debt are debt. Failure to make payment on an obligation that is not considered debt under this definition (for example, payment on delivery) will not give rise to debt.

11. Debt is considered concessional if, on the date on which the contract was signed, the ratio of the present value of the loan, based on the commercial interest reference rates (CIRRs), to the nominal value of the loan is less than 65 percent (that is, the grant element of the loan is at least equal to 35 percent of its nominal value). The present value of the loan will be calculated by discounting future payments of interest and principal using the CIRRs established by the Organization for Economic Cooperation and Development (OECD). Specifically, the 10-year average of CIRRs reported by the OECD will be used for loans with maturities longer than 15 years while the six-month average of CIRRs will be used for loans with shorter maturities. To both the 10-year and 6-month averages of the reference rate, the margin for different repayment periods will be added, as established by the OECD 
( 0.75 percent for repayment periods of less than 15 years; 1 percent for repayment periods of 15-19 years; 1.15 percent for repayment periods of 20-29 years; and 1.25 percent for repayment periods of 30 years or more).

12. The definition of external debt, for the purposes of the program, is any debt as defined in paragraph 9, which is denominated in foreign currency, i.e., currency other than Kenyan shillings (Ksh). Similarly, external borrowing is borrowing denominated in foreign currency.

13. The performance criterion on nonconcessional external debt contracted or guaranteed by the central government is measured as a cumulative flow of nonconcessional external debt contracted or guaranteed by the central government. The ceiling on non concessional external borrowing will exclude the proceeds of the issuance of a sovereign bond up to a maximum amount of US\$500 million. Medium- and long-term debt refers to debt with maturity of one year or longer.

\section{Performance Criterion on the Stock of Central Government and Central Government Guaranteed External Payment Arrears to Official Creditors}

14. Central government and central government guaranteed external payment arrears to official creditors are defined as overdue payments (principal or interest), which were not made by their contract due date nor during the applicable grace period thereafter, on debt contracted or guaranteed by the central government. The definition excludes arrears relating to debt subject to renegotiation (dispute) or rescheduling.

15. The performance criterion on the stock of central government and central government guaranteed external payment arrears applies only to newly accumulated arrears on or after December 31, 2010.

\section{Indicative Target on Priority Social SPENDING}

16. The program sets a floor on priority social spending of the central government. For the purposes of the program, priority social spending of the government is defined as the sum of:

- $\quad$ cash transfers to orphans and vulnerable children;

- $\quad$ cash transfers to elderly;

- anti-retroviral treatment expenditures;

- $\quad$ free primary education expenditure; and

- $\quad$ free secondary education expenditure. 


\section{Coverage}

17. All the references to central government in the current TMU will be changed to general government, in order to include local governments whenever the fiscal decentralization takes place according to the new constitution.

\section{Table 1. Kenya: Program Exchange Rates for the Extended Credit Facility}

(Rates as of September 1, 2010)

\begin{tabular}{lcc}
\hline & $\begin{array}{c}\text { Kenyan Shillings } \\
\text { Per Curreny Unit }\end{array}$ & $\begin{array}{c}\text { Dollars } \\
\text { Per Curreny Unit }\end{array}$ \\
\hline Australian dollar & 72.88 & 0.9000 \\
Canadian dollar & 77.14 & 0.9526 \\
Chinese yuan & 11.89 & 0.1468 \\
Danish krone & 13.92 & 0.1719 \\
Euro & 103.65 & 1.2800 \\
Japanese yen & 0.96 & 0.0118 \\
Norwegian krone & 13.05 & 0.1612 \\
Pound sterling & 124.65 & 1.5394 \\
Swedish krone & 10.99 & 0.1358 \\
SDR & 122.65 & 1.5147 \\
U.S. dollar & 81.00 & 1.0000 \\
\hline
\end{tabular}

Table 2. Kenya: External Budget Support to the Central Government 2010-11 1/

(In millions of U.S. dollars)

\begin{tabular}{lcrrr}
\hline & 2010 & & 2011 \\
\cline { 3 - 5 } & End-December & End-March & End-June & End-December \\
\hline & & & & 0.0 \\
External Budget Grants & 0.0 & 0.0 & 0.0 & 100.0 \\
External Budget Concessional Loans & 0.0 & 0.0 & 0.0 & 0.0 \\
External Budget Commercial Loans & 0.0 & 0.0 & 0.0 & 100.0 \\
Total External Budgetary Support & 0.0 & 0.0 & 0.0 \\
\hline
\end{tabular}

Source: Kenyan authorities data.

1/ For end-December, 2010, end-March 2011 and end-June 2011, the programmed amounts are cumulative from July 1 , 2010. For end-December 2011, the programmed amounts are cumulative from July 1, 2011. 
Table 3. Summary of Data to Be Reported

\begin{tabular}{|c|c|c|c|}
\hline Information & Frequency & Reporting Deadline & $\begin{array}{l}\text { Responsible } \\
\text { Entity }\end{array}$ \\
\hline \multicolumn{4}{|l|}{$\begin{array}{l}\text { 1. Primary balance of the central government } \\
\text { including grants }\end{array}$} \\
\hline $\begin{array}{l}\text { Net domestic bank financing (including net } \\
\text { commercial bank credit to the central government } \\
\text { and net CBK credit to the central government) }\end{array}$ & Monthly & $\begin{array}{l}\text { Within } 15 \text { days after } \\
\text { the end of the month. }\end{array}$ & CBK \\
\hline Net nonbank financing & Monthly & $\begin{array}{l}\text { Within } 15 \text { days after } \\
\text { the end of the month. }\end{array}$ & CBK \\
\hline $\begin{array}{l}\text { Central government arrears accumulation to domestic } \\
\text { private parties and public enterprises outstanding for } \\
60 \text { days or longer. }\end{array}$ & Monthly & $\begin{array}{l}\text { Within } 15 \text { days after } \\
\text { the end of the month. }\end{array}$ & $\begin{array}{l}\text { Ministry of } \\
\text { Finance (MoF) }\end{array}$ \\
\hline Proceeds from privatization & Monthly & $\begin{array}{l}\text { Within } 15 \text { days after } \\
\text { the end of the month. }\end{array}$ & CBK \\
\hline Interest paid on domestic debt & Monthly & $\begin{array}{l}\text { Within } 15 \text { days after } \\
\text { the end of the month. }\end{array}$ & CBK \\
\hline Interest paid on external debt & Quarterly & $\begin{array}{l}\text { Within } 4 \text { weeks after } \\
\text { the end of the quarter. }\end{array}$ & CBK \\
\hline $\begin{array}{l}\text { Disbursements of external nonconcessional project } \\
\text { loans, including securitization }\end{array}$ & Quarterly & $\begin{array}{l}\text { Within } 45 \text { days after } \\
\text { the end of the quarter. }\end{array}$ & $\mathrm{MoF}$ \\
\hline Disbursements of budget support loans & Quarterly & $\begin{array}{l}\text { Within } 45 \text { days after } \\
\text { the end of the quarter. }\end{array}$ & MoF \\
\hline Principal repayments on all external loans & Monthly & $\begin{array}{l}\text { Within } 15 \text { days after } \\
\text { the end of the month. }\end{array}$ & CBK \\
\hline Net proceeds from issuance of external debt & Monthly & $\begin{array}{l}\text { Within } 15 \text { days after } \\
\text { the end of the month. }\end{array}$ & CBK \\
\hline $\begin{array}{l}\text { Any exceptional financing (including rescheduled } \\
\text { principal and interest) }\end{array}$ & Monthly & $\begin{array}{l}\text { Within } 15 \text { days after } \\
\text { the end of the month. }\end{array}$ & $\mathrm{MoF}$ \\
\hline Net changes in the stock of short-term external debt & Quarterly & $\begin{array}{l}\text { Within } 45 \text { days after } \\
\text { the end of the quarter. }\end{array}$ & $\mathrm{MoF}$ \\
\hline Net change in external arrears, including interest & Quarterly & $\begin{array}{l}\text { Within } 45 \text { days after } \\
\text { the end of the quarter. }\end{array}$ & MoF \\
\hline \multicolumn{4}{|l|}{ 2. Gross official international reserves } \\
\hline $\begin{array}{l}\text { CBK's holding of monetary gold (excluding amounts } \\
\text { pledged as collateral) }\end{array}$ & Monthly & $\begin{array}{l}\text { Within } 15 \text { days after } \\
\text { the end of the month. }\end{array}$ & CBK \\
\hline Holdings of SDRs & Monthly & $\begin{array}{l}\text { Within } 15 \text { days after } \\
\text { the end of the month. }\end{array}$ & CBK \\
\hline $\begin{array}{l}\text { CBK holdings of convertible currencies in cash or in } \\
\text { nonresident financial institutions (deposits, securities, } \\
\text { or other financial instruments) }\end{array}$ & Monthly & $\begin{array}{l}\text { Within } 15 \text { days after } \\
\text { the end of the month. }\end{array}$ & CBK \\
\hline
\end{tabular}




\begin{tabular}{|c|c|c|c|}
\hline 3. Official reserve liabilities & & & \\
\hline $\begin{array}{l}\text { Total outstanding liabilities of the CBK to the IMF } \\
\text { except those arising from the August } 28,2009 \text { SDR } \\
\text { general allocation and the September 9,2009 SDR } \\
\text { special allocation; }\end{array}$ & Monthly & $\begin{array}{l}\text { Within } 15 \text { days after } \\
\text { the end of the month. }\end{array}$ & CBK \\
\hline $\begin{array}{l}\text { Convertible currency liabilities of the CBK to } \\
\text { nonresidents with an original maturity of up to and } \\
\text { including one year }\end{array}$ & Monthly & $\begin{array}{l}\text { Within } 15 \text { days after } \\
\text { the end of the month. }\end{array}$ & CBK \\
\hline $\begin{array}{l}\text { Commitments to sell foreign exchange arising from } \\
\text { derivatives. }\end{array}$ & Monthly & $\begin{array}{l}\text { Within } 15 \text { days after } \\
\text { the end of the month. }\end{array}$ & CBK \\
\hline \multicolumn{4}{|l|}{ 4. Net domestic assets } \\
\hline Net CBK credit to the central government & Monthly & $\begin{array}{l}\text { Within } 15 \text { days after } \\
\text { the end of the month. }\end{array}$ & CBK \\
\hline $\begin{array}{l}\text { Outstanding net CBK credit to domestic banks } \\
\text { (including overdrafts) }\end{array}$ & Monthly & $\begin{array}{l}\text { Within } 15 \text { days after } \\
\text { the end of the month. }\end{array}$ & CBK \\
\hline \multicolumn{4}{|l|}{ 5. Reserve money } \\
\hline Currency in circulation & Monthly & $\begin{array}{l}\text { Within } 15 \text { days after } \\
\text { the end of the month. }\end{array}$ & CBK \\
\hline Required and excess reserves & Monthly & $\begin{array}{l}\text { Within } 15 \text { days after } \\
\text { the end of the month. }\end{array}$ & CBK \\
\hline $\begin{array}{l}\text { Nonconcessional medium- and long-term external } \\
\text { debt contracted or guaranteed by the central } \\
\text { government }\end{array}$ & Quarterly & $\begin{array}{l}\text { Within } 45 \text { days after } \\
\text { the end of the quarter. }\end{array}$ & $\mathrm{MoF}$ \\
\hline $\begin{array}{l}\text { Accumulation of central government and central } \\
\text { government guaranteed external payment arrears. }\end{array}$ & Quarterly & $\begin{array}{l}\text { Within } 45 \text { days after } \\
\text { the end of the quarter. }\end{array}$ & $\mathrm{MoF}$ \\
\hline Social priority spending & Quarterly & $\begin{array}{l}\text { Within } 45 \text { days after } \\
\text { the end of the quarter. }\end{array}$ & MoF \\
\hline
\end{tabular}




\section{INTERNATIONAL MONETARY FUND}

\section{KENYA}

\section{Request for a Three-Year Arrangement Under the Extended Credit Facility Informational Annex}

Prepared by the African Department

Approved by Saul Lizondo and Dominique Desruelle

January 14, 2011

Contents

Page

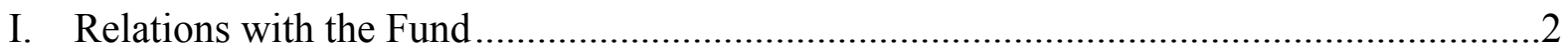

II. World Bank and IMF Collaboration-JMAP Implementation 2010-11 _......................6

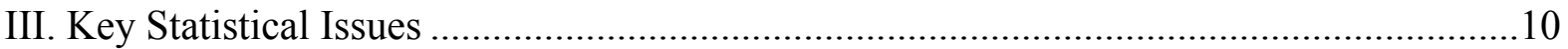




\section{KENYA: RELATIONS WITH THE FUND}

(As of October 31, 2010)

I. Membership Status: Joined February 3, 1964; Article VIII.

\section{General Resources Account:}

Quota

Fund holdings of currency

Reserve Tranche Position

III. SDR Department:

Net cumulative allocation

Holdings

IV. Outstanding Purchases and Loans:

ESF RAC Loan

ECF Arrangements
SDR million

271.40

258.47

12.96

SDR million

259.65

209.34

SDR million

135.70

137.50
Percent of quota

100.00

95.24

4.78

Percent of allocation

100.00

80.62

Percent of quota

50.00

50.66

\section{Latest Financial Arrangements:}

$\begin{array}{lrrrr}\text { Type } & \begin{array}{r}\text { Approval } \\ \text { Date }\end{array} & \begin{array}{r}\text { Expiration } \\ \text { Date }\end{array} & \begin{array}{r}\text { Amount } \\ \text { Approved } \\ \text { (SDR Million) }\end{array} & \begin{array}{r}\text { Amount Drawn } \\ \text { (SDR Million) }\end{array} \\ \mathrm{ECF}^{1} & \underline{\underline{\text { SDR }}} & & & \\ \mathrm{ECF}^{1} & 11 / 21 / 03 & 11 / 20 / 07 & 150.00 & 150.00 \\ \mathrm{ECF}^{1} & 08 / 4 / 00 & 08 / 3 / 03 & 190.00 & 33.60 \\ & 04 / 26 / 96 & 04 / 25 / 99 & 149.55 & 24.93\end{array}$

${ }^{1}$ Former PRGF

The last three-year PRGF arrangement was approved on November 21, 2003, in the amount of SDR 175 million. Access was augmented to SDR 225 million at the time of the first review on December 20,2004, to address the impact of a drought and higher oil prices. In light of a balance of payments improvement and at the request of the authorities, access was reduced to SDR 150 million at the time of the second review. The arrangement expired on November 20, 2007. In May 2009, the Executive Board approved the disbursement of 
SDR135.7 under the RAC-ESF to address the impact of exogenous shocks and the balance of payments gap.

\section{Projected Obligations to Fund ${ }^{2}$}

(SDR million; based on existing use of resources and present holdings of SDRs):

\begin{tabular}{lrrrrr}
\hline & $\frac{2010}{2.50}$ & $\frac{2011}{15.00}$ & $\frac{2012}{18.75}$ & $\frac{2012}{30.00}$ & $\frac{2013}{38.57}$ \\
$\begin{array}{l}\text { Principal } \\
\text { Charges/interest } \\
\text { Total }\end{array}$ & $\underline{0.04}$ & $\underline{0.20}$ & $\underline{0.82}$ & $\underline{0.76}$ & $\underline{0.69}$ \\
\hline 2 when a member has overdue financial obligations outstanding for more than three months, the \\
amounts of such arrears will be shown in this section
\end{tabular}

VII. Implementation of HIPC Initiative: Not Applicable

VIII. Implementation of Multilateral Debt Relief Initiative (MDRI): Not Applicable

\section{Safeguards Assessments}

An update safeguards assessment of the CBK took place in Spring 2009 with respect to the authorities request for a disbursement under the Rapid-Access Component of the ESF. The update assessment found increased safeguards risk at the CBK since the 2005 assessment. The report noted that deficiencies found in recent external audits and noncompliance with the benchmark financial reporting framework confirmed that audit oversight is not yet fully effective, and that it has impaired external audit and financial reporting. Steps were taken by the CBK to strengthen the 2009/10 external audit process.

\section{Exchange Arrangements}

Kenya's currency is the shilling, which floats against other currencies. The official exchange rate, which is set at the previous day's average market rate, applies only to government and government-guaranteed external debt-service payments and to government imports for which there is a specific budget allocation. The exchange rate regime is a float, in which the U.S. dollar is the principal intervention currency. Kenya has accepted the obligations of Article VIII, Sections 2, 3, and 4, and maintains an exchange system free of restrictions on payments and transfers for current international transactions, other than restrictions notified to the Fund under Decision No. 144 (52/51). On November 30, 2010, the exchange rate was KSh $80.97=\mathrm{US} \$ 1.00$. 


\section{Article IV Consultations}

The last Article IV consultation with Kenya concluded on December 7, 2009. Kenya is subject to a 12-month consultation cycle.

\section{FSAP Participation}

A joint IMF/WB mission assessed Kenya's financial sector as part of the Financial Sector Assessment Program (FSAP) update during September 2009. The staff report on the Financial Sector Stability Assessment was issued to the Executive Board concurrently with the 2009 Article IV Consultation Staff Report.

\section{Technical Assistance}

\begin{tabular}{|c|c|c|}
\hline Department & Purpose & Time of Delivery \\
\hline STA & ROSC Mission & January 2005 \\
\hline MFD & $\begin{array}{l}\text { Monetary Framework, Monetary Operations, } \\
\text { and Banking Supervision }\end{array}$ & October 2005 \\
\hline FAD & Revenue Administration & November 2005 \\
\hline FAD & $\begin{array}{l}\text { AFRITAC East Semi-Autonomous Agencies } \\
\text { and EBFs/Fiscal Reporting Mission }\end{array}$ & March 2006 \\
\hline FAD & $\begin{array}{l}\text { HQ Mission: Strengthening the Budget and } \\
\text { Reporting System }\end{array}$ & August 2006 \\
\hline FAD & $\begin{array}{l}\text { AFRITAC East TA: Revision of Public } \\
\text { Finance Management Act }\end{array}$ & October/November 2006 \\
\hline $\mathrm{MCM}$ & $\begin{array}{l}\text { AFRITAC East TA: Review of Risk-Based } \\
\text { Supervision Manual }\end{array}$ & November/December 2006 \\
\hline MCM & Monetary Operations TA & January 2007 \\
\hline STA & External Debt Statistics Workshop & February / March 2007 \\
\hline FAD & AFRITAC East TA: Customs Administration & July 2007 \\
\hline FAD & Fiscal ROSC & July 2007 \\
\hline MCM & Payment Systems & February 2008 \\
\hline STA & Consumer Price Statistics & March 2008 \\
\hline $\mathrm{MCM}$ & Monetary Operations/Banking & April 2008 \\
\hline STA & National Accounts & April 2008 \\
\hline FAD & AFRITAC East TA: Budget & May 2008 \\
\hline STA/MCM & Financial Soundness Indicators & June 2008 \\
\hline FAD & Budgeting & July 2008 \\
\hline MCM & Bond-Pricing Techniques Workshop & July 2008 \\
\hline FAD & $\begin{array}{l}\text { AFRITAC East TA: Regional PFM Advisor: } \\
\text { Budget and Tax review }\end{array}$ & August 2008 \\
\hline FAD & AFRITAC East TA: Customs Administration & \\
\hline MCM & Payment Systems & August 2008 \\
\hline STA & $\begin{array}{l}\text { AFRITAC East TA: Monetary and Financial } \\
\text { Statistics }\end{array}$ & 2008 \\
\hline FAD & Tax Administration Workshop & November 2008 \\
\hline
\end{tabular}




\begin{tabular}{|c|c|c|}
\hline FAD & PFM and Fiscal Decentralization & November 2008 \\
\hline STA & Reweighing and Rebasing of CPI & December 2008 \\
\hline STA & Producer Price Index compilation & December 2008 \\
\hline MCM & Risk based Supervision & January 2009 \\
\hline MCM & Capital Market Regulation and Supervision & March 2008 \\
\hline FAD & $\begin{array}{l}\text { Several missions on fiscal decentralization, } \\
\text { customs administration, and public finance } \\
\text { management }\end{array}$ & $2009-2010$ \\
\hline \multirow[t]{2}{*}{ FAD } & PFM mission on Strengthening Program & \\
\hline & Budgeting & July 2010 \\
\hline FAD & Several missions on Customs Administration & 2010 \\
\hline \multirow[t]{2}{*}{ FAD } & PFM mission on IFMIS and budget module & \\
\hline & Preparation & September 2010 \\
\hline FAD & Several other review missions on PFM & January 2011 \\
\hline \multirow[t]{2}{*}{ LEG } & Missions on Anti-Money Laundering & \\
\hline & legislation and the Banking Law & 2010 \\
\hline $\mathrm{MCM}$ & Mission on asset and liability management & May 2010 \\
\hline MCM & Aligning off-site Surveillance & August 2010 \\
\hline MCM & Mission to evaluate financial stability & January 2011 \\
\hline MCM & Mission on insurance and pension supervision & February 2011 \\
\hline MCM & $\begin{array}{l}\text { Conducting onsite inspection and } \\
\text { consolidated supervision }\end{array}$ & February 2011 \\
\hline $\mathrm{MCM}$ & $\begin{array}{l}\text { Several mission on monetary operations, bank } \\
\text { supervision, payments system and capital } \\
\text { markets development }\end{array}$ & $2009-2010$ \\
\hline STA & $\begin{array}{l}\text { Several missions on national accounts, CPI, } \\
\text { and external sector statistics }\end{array}$ & $2009-2010$ \\
\hline STA & Consumer price Indices & May 2010 \\
\hline STA & Train staff on PPI Method & May 2010 \\
\hline STA & Assist with producing quarterly GDP & October 2010 \\
\hline STA & Mission on balance of payments & \\
\hline STA & $\begin{array}{l}\text { statistics (DFID III) } \\
\text { DFID III follow-up GFS to implement }\end{array}$ & $\begin{array}{l}\text { November } 2010 \\
\text { January } 2011\end{array}$ \\
\hline
\end{tabular}

\section{Resident Representative}

Mr. Ragnar Gudmundsson, since August 2010. 


\section{KenYa: Joint WB-IMF MANAgement ACTiOn Plan OCTOBER 2010-SePtember 2011}

1. The Fund Kenya team led by Mr. Fanizza (mission chief) met with the World Bank Kenya team led by Mr. Zutt (country director) on September 21, 2010 to identify macro critical structural reforms and coordinate the two teams' work for the period October 2010-September 2011.

2. The teams agreed that Kenya's main macroeconomic challenges are to ensure public debt sustainability while strengthening the investment climate to diversify the economy. To meet these challenges, Kenya needs to consolidate its fiscal position over the medium term to bring the public debt-to-GDP ratio back to about 45 percent while creating the right environment for investments to unlock the country's growth potential.

3. Based on this shared assessment, the teams identified the following structural reform areas as macro critical, as they will be key to achieving sustained growth, a strengthening of the fiscal position, and debt sustainability:

- Public financial management reform: Progress has been made recently in linking expenditure to medium term policy objectives. However, the preparation, execution, and monitoring of the budget need to be strengthened. This is important in light of the planned devolution of at least fifteen percent of government revenues to local levels of government by 2015 . Key elements of a reform include (i) supporting the complete roll out of IFMIS in all ministries through the Institutional Reform and Capacity Building project; (ii) establishing a Single Treasury Account to improve Treasury control; and (iii) implementing a comprehensive Public Finance Administration bill prior to the transfer of resources to decentralized units.

- Tax reform: Efforts are under way to enhance tax policy and administration, and will center on the implementation of a new VAT to be modernized and simplified in line with best practice. Customs administration is also set to be strengthened in line with commitments under the EAC Common Market Protocol.

- Financial sector reform: The ongoing modernization of the financial system and the growth in the regional activities of financial institutions call for enhanced consolidated and cross-border supervision as well as an upgrade in the crisis management framework. Focus also needs to be placed on strengthening Kenya's capital markets through the demutualization of the stock exchange, enhancing the Capital Markets Authority and further development of debt and capital markets in the regional context. Similar efforts are required to strengthen insurance markets, highlighted by the FSAP update as one of the weaker areas in the system. An additional weak area highlighted by the FSAP update is related to the NSSF and other sustainability aspects of the public pension system. 
- Infrastructure reform: Infrastructure has been identified as one of the most problematic factors for doing business in Kenya. Reforms focus in three specific areas (i) improvements at the Mombasa port; (ii) strengthening Kenya's infrastructure in transportation, energy, water and sanitation; and (iii) improving PPP institutional capacity at the ministries.

- Investment climate reform: Kenya has made important progress in improving the investment climate. Nonetheless, other countries have been reforming even faster, and post-election violence slowed down the reform momentum in Kenya. The slack in reforms partly explains Kenya's (relative) regression in international rankings of investment climate. The authorities need to revive the reform momentum to create a better investment climate, both through interventions in private sector competitiveness as well as financial sector deepening and strengthening.

\section{The teams agreed on the following division of labor:}

- Public financial management reform: The Fund is providing technical assistance (TA) on a comprehensive Public Financial Management bill, on program budgeting, as well as support on the establishment of a Single Treasury Account. The WB is supporting the implementation of IFMIS and essential PFM reforms through the Institutional Reform and Capacity Building Project and proposed DPL.

- Tax reform: The Fund is providing technical assistance on enhancing customs administration, including through improvements to inspection procedures and information technology.

- Public Expenditure Review: The WB will lead in the PER with specific focus on service delivery in agriculture education and health sectors and building capacity for social accountability, including expenditure tracking and public expenditure analysis.

- Financial sector reform: Reform efforts are being supported by the WB through the Financial and Legal Sector Technical Assistance Loan (FLSTAP) since 2005 with a strong agenda on all regulatory and supervisory agents in the market. Support from the Fund has been through technical assistance on focalized aspects such as stress testing. The WB and the Fund should coordinate technical assistance on the establishment of a crisis management framework, per discussions prior and during the FSAP update. Efforts should also be coordinated on pension markets (particularly with regard to the NSSF). Ad-hoc support related to the insurance sector should be carefully linked to ongoing reform effort through the FLSTAP.

- Infrastructure reform: The WB has been supporting the developments related to PPPs and infrastructure finance for the last few years, intensified early this year with the creation of the PPP secretariat. The authorities have requested support for the growing 
agenda, including through the preparation of a new PPP/infrastructure finance project. Additional elements are emerging, such as contingent liabilities analysis related to PPP and beyond, which will be coordinated with the Fund.

- Investment climate reform: The WB has been supporting investment climate reforms through the FLSTAP (i.e., support for the drafting, peer reviewing, and sharing of international practices on key laws; reforms to companies and land registries, and legal framework for lending) which the proposed DPL is expected to complement. It also supports targeted interventions related to business environment through the Kenya investment climate program and the micro, small, and medium-sized enterprises (MSME) competitiveness project. Analytical work on industry mapping/clusters is also ongoing.

- Fiscal decentralization: The WB and Fund will jointly provide TA on fiscal decentralization envisaged in the new constitution. The TA will focus on setting up equitable and efficient fiscal and public finance management systems and mechanisms that allow county governments to perform their functions effectively over the long term.

- ECF arrangement with the Fund: ECF-supported program will focus on strengthening Kenya's external position, promoting gradual fiscal consolidation while stepping up infrastructure investment and providing sufficient resources to implement the new constitution, bringing back the debt burden to the government's medium-term target, maintaining the floating exchange rate regime, and moving gradually to an inflation targeting framework. The program will seek to strengthen public financial management, modernize and simplify tax policy, and broaden access to financial services.

4. The teams have the following requests for information from their counterparts:

- $\quad$ The Fund team requests to be kept informed of progress in the above macro critical structural reform areas. Timing: when milestones are reached (and at least semiannually).

- $\quad$ The WB team requests to be kept informed of the Fund's assessments of macroeconomic policies and prospects. Timing: in the context of Article IV and other missions (and at least semi-annually).

5. The table below lists the teams' separate and joint work programs during October 2010-September 2011.

\begin{tabular}{|c|c|c|c|}
\hline Title & Products & $\begin{array}{c}\text { Provisional Timing of } \\
\text { Mission }\end{array}$ & $\begin{array}{c}\text { Expected Delivery } \\
\text { Dates }\end{array}$ \\
\hline $\begin{array}{l}\text { 1. WB Work } \\
\text { Program }\end{array}$ & $\begin{array}{l}\text { Kenya economic update } \\
\text { (bi-annually) } \\
\text { Institutional reform and } \\
\text { Capacity building: which has }\end{array}$ & $\begin{array}{l}\mathrm{N} / \mathrm{A} \\
\mathrm{N} / \mathrm{A}\end{array}$ & $\begin{array}{l}\text { Dec. } 2010 \text { \& June } 2011 \\
\text { Continuous }\end{array}$ \\
\hline
\end{tabular}




\begin{tabular}{|c|c|c|c|}
\hline & $\begin{array}{l}\text { two components (i) PFM; } \\
\text { (ii) institutional reform and } \\
\text { capacity building } \\
\text { Public expenditure review } \\
\text { Development Policy Loan } \\
\text { (DPL) } \\
\text { PPPs/Infrastructure Finance } \\
\text { FLSTAP_financial regulators } \\
\text { and market development (i.e., } \\
\text { insurance, banking, capital } \\
\text { markets); housing finance. } \\
\text { analysis } \\
\text { MSME, KICP, analytical work } \\
\text { on investment climate }\end{array}$ & $\begin{array}{l}\text { November } 2010 \\
\text { TBD } \\
\text { N/A } \\
\text { N/A }\end{array}$ & $\begin{array}{l}\text { April } 2011 \\
\text { July } 2011 \\
\text { July } 2011 \\
\text { Continuous } \\
\text { Continuous }\end{array}$ \\
\hline $\begin{array}{ll}\text { 2. } & \text { Fund } \\
\text { Work } \\
\text { Program }\end{array}$ & $\begin{array}{l}\text { Missions } \\
\text { Staff mission for negotiations } \\
\text { on a financing arrangement } \\
\text { Article IV consultation } \\
\text { TA provision on fiscal } \\
\text { regulations; program } \\
\text { budgeting; fiscal } \\
\text { decentralization; IFMIS and } \\
\text { treasury single account support } \\
\text { TA provision on customs } \\
\text { administration and modern } \\
\text { risk management } \\
\text { TA provision on crisis } \\
\text { management framework }\end{array}$ & $\begin{array}{l}\text { November } 2010 \\
\text { To be determined } \\
\text { (TBD) } \\
\text { Ongoing } \\
\text { November } 2010 \\
\text { November } 2010\end{array}$ & $\begin{array}{l}\text { January } 2011 \\
\text { TBD } \\
\text { Implementation } \\
\text { throughout fiscal year } \\
2011 \\
\text { TA report in February } \\
2011 \\
\text { January } 2011\end{array}$ \\
\hline $\begin{array}{ll}\text { 3. } & \text { Joint } \\
\text { Work } \\
\text { Program }\end{array}$ & $\begin{array}{l}\text { Various TA provisions on } \\
\text { PFM reforms, fiscal } \\
\text { decentralization, crisis } \\
\text { management framework } \\
\text { (FLSTAP, FIRST) and } \\
\text { Pensions (NSSF and } \\
\text { contingent liabilities) } \\
\text { Public and external debt } \\
\text { Sustainability Analysis }\end{array}$ & $\begin{array}{l}\text { December } 2010 \text { as part } \\
\text { of the DPL and } \\
\text { continuous } \\
\text { November } 2010\end{array}$ & January 2011 \\
\hline
\end{tabular}




\section{KENYA: Key STATISTICAL ISSUES}

(As of January 10, 2011)

\section{A. Assessment of Data Adequacy for Surveillance}

\section{General}

1. Data provision has some shortcomings, but is broadly adequate for surveillance and program monitoring. Further improvements in the methodology of compiling real, fiscal, and external sector statistics would be desirable in order to facilitate enhanced design and monitoring of economic policies. However, the overall quality, timeliness, and coverage of macroeconomic statistics have significantly improved over the past few years. The Fund has substantially facilitated this process through technical assistance from the Statistics Department, the Fiscal Affairs Department, and the Monetary and Capital Markets Department. Monetary, exchange rate, and some external data are published on a monthly and biannual basis by the Kenya National Bureau of Statistics (KNBS), and by the CBK in its Monthly Economic Review. Core financial data are also made available to the Fund on a regular basis. A detailed account of various sectoral activities and the corresponding statistical data are published annually by the KNBS in its Economic Survey.

\section{National accounts}

2. The KNBS has made significant efforts to enhance national statistics compilation. Annual national accounts estimates for the years 1996-2008 in current and constant (2001 prices) have been published both from the expenditure and production approaches. The KNBS has made significant progress in developing estimates of quarterly GDP at constant prices that are now published. However, problems remain including estimates of the informal sector, use of deflators, coverage of electricity generation, mining/petroleum exploration, private water supply and sewerage, and excessive use of fixed ratios for estimates.

\section{Price statistics}

3. The KNBS still faces significant challenges in it is compilation of price indices. In particular, the compilation and dissemination of a CPI series with a historical dimension. In early 2002, the KNBS (then CBS) began publication of a new national CPI (covering 13 urban towns), with 1997 as reference year and outdated weights and basket of items derived from the 1993-94 Household Budget Survey. Indices are produced for lower and middle/upper income groups in Nairobi and other cities. In March 2008, a TA mission assisted the authorities in identifying the methodological issues relating to the measurement of inflation. The current methodology of aggregating prices at the elementary level was found to impart a substantial upward bias on CPI measurement. In line with STA recommendations, the KNBS released in November 2009 a revised series that corrected the elementary aggregation problem. In February 2010, the KNBS rebased the series. The current series starts in February 2009 and the KNBS only plans to publish a historical series 
based on this methodology in 2012. The KNBS does not compile producer, export, or import price indices.

\section{Government finance statistics}

4. Since the beginning of FY 2005/06, Kenya has followed a new economic classification of the budget based on the GFSM 2001. The migration to GFSM 2001 may help reduce the significant differences between KNBS data and the data compiled by the $\mathrm{MoF}$ and reported to AFR for surveillance and program monitoring purposes. However, serious delays have emerged in reporting, reflecting difficulties in establishing budget execution and accounting systems consistent with the new classification. To ensure timely reconciliation and monitoring of budget execution, the existing systems would need to be promptly upgraded and made operational. Progress has been made toward moving to the IFMIS. There are still important gaps in ensuring proper reconciliation of fiscal data from different sources, including from various units within the MoF. The discrepancies in budget outturn data (between deficit/surplus and financing) remain significant, and the recording of external financing and expenditure directly financed from abroad is still an important area for improvement. The government has taken some steps to initiate a project to rationalize/ eliminate extrabudgetary funds, but the progress in compiling consolidated fiscal statistics has remained limited.

\section{Monetary statistics}

5. Progress has been shown in the implementation of the Monetary and Financial Statistics Manual and development of the SRFs. A major issue for future work relates to the expansion of the coverage of the ODCs to include the Savings and Credit Cooperatives (SACCOS). It is estimated that about 3,800 SACCOs operate in Kenya, accounting for about 30 percent of the total deposits of the banking system. When implemented, a new bill providing for increased supervision over SACCOs would generate data as part of the supervisory function. In addition to SACCOs, ODCs coverage needs to be expanded to include licensed microfinance institutions, the Post Office Savings Bank, deposit taking investment banks, and mutual funds. Kenya will benefit from participating in the monetary module of the DFID/Enhanced Data Dissemination Initiative (EDDI) project for Africa. The first mission under this project will take place in April 2011.

\section{Balance of payments}

6. The KNBS compiles annual balance of payments statistics in Kenya shillings, following the Fifth Edition of the Balance of Payments Manual (BPM5) classifications. The financial account is largely drawn from the International Transactions Reporting System (ITRS) which suffers from a large and growing element of unclassified inflows and outflows that might well include settlements of current account transactions. 
7. Kenya participates in the external sector module of the EDDI Project. Three TA missions have been conducted under the auspices of this project to undertake a comprehensive enterprise survey of cross-border financial flows and stocks with a view to significantly improving the quality of balance of payments (BOP) and to compile international investment position (IIP) statistics. The latest November 8-19, 2010 TA mission assessed the results of the 2009 IIP survey and the 2010 Foreign Investment Survey (2010 FIS), and designed the frame for a 2011 FIS.

8. The 2009 IIP Survey was conducted on a very limited budget and confined to the collection of position data needed for IIP compilation and did not cover related cross-border financial account transactions. The 2010 FIS was conducted with financial assistance from the World Bank Statistics Capacity Building project, to collect BOP data for 2008 and 2009 and related IIP data. This survey can be judged a success as an effective use of external resources, although the survey frame was smaller than planned.

9. The mission advised on steps needed to ensure that the 2011 FIS will meet the needs of the Fund's Coordinated Direct Investment Survey and the Sixth Edition of the Fund's Balance of Payments and International Investment Position Manual. However, a decision on the size of the frame will have to await clarification of available external funding and staff resource.

10. The establishment of a database developed by the Macroeconomic and Financial Management Institute of Eastern and Southern Africa (MEFMI) for use within the region for private capital flows surveys and for generating reports proved very effective. This database generates tables that are needed to review reported data to assess gaps and outliers. However, a number of additional tables should be added by MEFMI to the menu of output tables that are generated in the database.

11. Once established on an annual basis, the FIS will be a major data source in support of direct reporting and the integration of the balance of payments and IIP compilation, replacing the International Transactions Reporting System as a data source.

\section{B. Data Standards and Quality}

12. Kenya participates in the Fund's GDDS and the EDDI project for Anglophone Africa. A Data ROSC was conducted in October 2005.

\section{Reporting to STA}

13. The data for the budgetary central government, submitted for publication in the GFS Yearbook, have been reported in GFSM 2001 format, albeit with a significant lag. Monthly and quarterly GFS data are regularly reported for inclusion in the International Financial Statistics (IFS). Monetary data for the central bank and ODCs are regularly reported for inclusion in the IFS. Annual balance of payments data, following BPM5 classifications, are regularly reported to STA. 


\title{
INTERNATIONAL DEVELOPMENT ASSOCIATION
}

\author{
INTERNATIONAL MONETARY FUND
}

\author{
KENYA
}

\section{Joint IMF/World Bank Debt Sustainability Analysis}

\author{
Prepared by the Staffs of the International Monetary Fund and \\ the World Bank
}
Approved by Saul Lizondo and Dominique Desruelle (IMF) and Jeffrey Lewis and Jan Walliser (World Bank)

January 14, 2011

This analysis updates the May 2009 joint Bank/Fund debt sustainability analysis (DSA). ${ }^{1}$ Compared to the 2009 analysis (Country Report No. 09/191), debt sustainability indicators have deteriorated somewhat, reflecting a projected faster debt accumulation over the medium term. Risks are somewhat greater for public debt, particularly in the event of lower growth. Nevertheless, Kenya remains at low risk of external debt distress. ${ }^{2,3}$ The projected investment in infrastructure and the assumed improvement in the investment climate would be crucial to sustaining strong exports and GDP growth. Strategies to guard against shocks could include a build-up in international reserves as envisaged in the ECF framework.

\section{Background}

1. At end-2009, nominal public external debt was estimated at $\mathbf{\$ 7 . 1}$ billion (23\% $3 / 4$ percent of GDP). About 60 percent of this debt was to multilateral creditors (including 47 percent owed to the World Bank) and 39 percent to bilateral creditors. A small share (under 2 percent), owed to commercial creditors, represents disputed arrears on securityrelated contracts.

\footnotetext{
${ }^{1}$ It has benefited from consultation with African Development bank staff.

${ }^{2}$ Kenya still classifies as a medium performer in terms of the quality of its policies and institutions as measured by a three-year average of the World Bank's Country Policy and Institutional Assessment (CPIA) index. Available at http://go.worldbank.org/AXO6I14PK0.

${ }^{3}$ For a medium performer, the indicative thresholds for external debt sustainability are a net present value (NPV) of debt-to-GDP ratio of 40 percent, an NPV of debt-to-exports ratio of 150 percent, an NPV of debt-torevenue ratio of 250 percent, a debt service-to-exports ratio of 20 percent, and a debt service-to-revenue ratio of 30 percent.
} 
2. Kenya has managed its debt relatively well and has regularly met its obligations, except for some disputed commercial arrears. Limited external borrowing has left Kenya with more manageable debt ratios than many of its low-income country peers. Kenya benefited from Paris club rescheduling but did not qualify for heavily indebted poor countries (HIPC) debt relief as its debt indicators have been below the HIPC Initiative thresholds.

\section{The disputed external commercial arrears estimated at US\$242 million are a} subject of on-going investigations and litigation. The time-line for clearance of these arrears has not been determined. The amount of arrears has been revised upward from an earlier estimate of US\$91 million following completion of independent valuation of works, goods, and services delivered under each contract. The authorities think that it is more prudent to estimate a higher figure to reflect the likelihood of court rulings in favor of all creditors.

4. Kenya's net domestic debt stood at Ksh 584 billion at end-2009 $(20 \% / 4$ percent of GDP), but potential contingent liabilities could be very large. During 2003-07, domestic debt decline to $13 \frac{1}{2}$ percent of GDP, thanks to strong economic growth, prudent fiscal policies, and lower interest rates. The downward trend was reversed during 2008-09 reflecting fiscal stimulus measures implemented to mitigate the impact of adverse shocks. However, Kenya's relatively low reported domestic debt-to-GDP ratio masks vulnerabilities from possible realization of contingent liabilities associated with parastatals debt and unfunded obligations of the National Social Security Fund and government's current pay-asyou-go pension scheme for civil servants (equivalent to 11.8 percent of 2008/09 GDP).

5. The DSA is based on nonreconciled debt data provided by the authorities, available data on private sector debt, and staff estimates. It consists of two partsexternal and fiscal.

- $\quad$ The external DSA covers external debt of the central government (including parastatal borrowing with a government guarantee) and the central bank, and also includes estimates of private sector debt based on available information. External debt sustainability is assessed in relation to policy-dependent debt-burden thresholds. A single discount rate is used.

- The fiscal DSA covers total debt-external and domestic - incurred or guaranteed by the central government. ${ }^{4}$

\footnotetext{
${ }^{4}$ Public domestic debt comprises central government debt. In this analysis, total public debt refers to the sum of public domestic and public external debt, but does not cover the entire public sector (e.g., parastatal borrowing without a government guarantee is not covered).
} 


\section{Macroeconomic Assumptions}

\section{Key medium-term assumptions underlying the DSA are consistent with the Staff Report for Request for Access to Extended Credit Facility (ECF):}

- $\quad$ Real GDP growth is projected at 5 percent for 2010, a pickup from the average growth of 2.1 percent recorded during 2008-09, as a result of adverse shocks, including the global financial crisis. It is projected at an annual average of about

- $\quad 61 / 3$ percent during 2010-15, and just above 6 percent thereafter. While the projected growth exceeds the average of the past decade, it is not overly optimistic. It remains, on average, about the same as the pre-crisis five-year average of just above 6 percent and represents a deceleration from the 2007 growth of 7 percent. As such, the projected growth reflects in part a resumption of the momentum that was abruptly disrupted by the 2008 post-elections violence. ${ }^{5}$ Growth is also predicated on the improvement in road and energy infrastructure, the business climate, and productivity. It is expected that private investment will accelerate, taking advantage of lower energy costs and new opportunities in an expanded regional market.

- $\quad$ Average inflation of about 5 percent for 20011-15 as measured by the GDP deflator.

- A broadly constant real exchange rate is assumed during the medium term.

- $\quad$ The noninterest external current account deficit rises to about $71 / 4$ percent of GDP in 2010, before falling to about 3 percent of GDP by 2015 as the increase in the imports bill subsides and private transfers-to-GDP return to the pre-crisis levels.

- Assumptions in the fiscal area include broadly constant revenue and grants as a share of GDP (about 26 percent) ${ }^{6}$ domestically financed development spending gradually increasing from just about 6.0 percent of GDP in 2008 to about 6.8 percent by 2029; a constant wage bill of 7.1 percent of GDP, and a gradual decline in other recurrent spending from 8.9 percent of GDP in 2008 to 7.2 percent of GDP over the long-term in line with the government's budget strategy. The primary fiscal deficit was 3.7 percent of GDP in 2009/10 and gradually declines to 1.2 by the end of the forecast period.

\footnotetext{
${ }^{5}$ The likelihood of the domestic shocks of this nature has been reduced following the August 2010 ratification of the new constitution.

${ }^{6}$ The increase from the average of about 23 percent of GDP in the past three years reflect an expected improvement in the revenue mobilization effort stemming from tax reforms, as well as in the capacity to absorb project grants.
} 
- $\quad$ Real interest rates on domestic public debt are assumed at 3 percent for short-term debt and 5 percent for medium- and long-term debt.

- New domestic borrowings consist of a quarter of short-term debt and three-quarters of medium- and long-term debt, with the latter having an average maturity of about seven years. The NPV of domestic debt is assumed to be equal to its face value.

- New external borrowing as a share of GDP (including nonconcessional borrowing described below) increases over the medium and then declines gradually. It is projected to average $2 \frac{1}{2}$ percent of GDP during 2011-15, up from 2 percent of GDP in 2009. It subsequently declines, falling below 1 percent by the end of the forecast period. New external borrowing assumptions include sovereign bond issuance of $\$ 500$ million in 2012, additional commercial borrowing of about US\$450 million during 2013-14 and about US\$200 million per year in the long run. Assumptions on terms include a 71/2 percent fixed interest rate and a bullet amortization in year 10 .

7. Continued eligibility for concessional borrowing from the International Development Association is assumed, although achievement of assumed growth rates could imply graduation during the forecast period.

\section{External DebT Sustainability}

8. Kenya faces a low risk of external debt distress reflecting the limited reliance on external borrowing and an expected improvement in macroeconomic performance.

- $\quad$ Under the baseline scenario, initial debt ratios are well below all of the indicative thresholds for a medium performer, even if they increase over the medium-term reflecting a higher rate of debt accumulation (see Figure 1 and Table $2 a$ and $2 b$ ).

- $\quad$ Alternative scenarios and stress tests indicate that Kenya's external debt situation is generally resilient. Standard stress tests reveal an initial upward trend for the debt indicators but do not result in a breach of the thresholds during the projection period. Over the period 2011-15, a shock combining lower GDP growth, weaker exports, a lower GDP deflator, and a fall in nondebt creating flows would push the NPV of public external debt as a share of GDP from 181/4 percent to 25 percent, and the NPV of debt-to-exports from almost 66 percent to $961 / 3$ percent. The most extreme shocks to debt dynamics by 2020 generally stem from a one-time 30 percent depreciation in 2011 or from a one standard deviation shock to the growth of exports proceeds. ${ }^{7}$

\footnotetext{
${ }^{7}$ The most extreme shock to the NPV of debt-to-GDP, the NPV of debt-to-revenue, and debt service-to-revenue results from a 30 percent exchange rate depreciation in 2011, whereas the most extreme shock to the PV of debt-to-exports results from an exports growth subdued during 2011-12 at only 1.3 percent (the historical average minus one standard deviation).
} 
Summary: External Debt Sustainability Assessment

\begin{tabular}{|c|c|c|c|c|c|c|}
\hline \multicolumn{7}{|c|}{ (In percent of GDP) } \\
\hline & 2010 & 2011 & 2012 & 2013 & 2014 & 2015 \\
\hline \multicolumn{7}{|c|}{$\begin{array}{l}\text { NPV of PPG External Debt } \\
\text { In percent of GDP (threshold=40) }\end{array}$} \\
\hline Baseline & 18.2 & 18.2 & 19.3 & 19.6 & 19.4 & 18.9 \\
\hline Combined shocks & 18.2 & 19.8 & 26.4 & 26.4 & 25.9 & 25.0 \\
\hline \multicolumn{7}{|c|}{ In percent of exports (threshold=150) } \\
\hline Baseline & 66.0 & 69.2 & 70.9 & 73.2 & 77.8 & 74.7 \\
\hline Combined shocks & 66.0 & 71.5 & 94.7 & 96.2 & 101.1 & 96.3 \\
\hline \multicolumn{7}{|c|}{$\begin{array}{l}\text { PPG External Debt Service } \\
\text { In percent of exports (threshold=20) }\end{array}$} \\
\hline Baseline & 4.1 & 4.1 & 3.9 & 4.0 & 4.4 & 4.2 \\
\hline Combined shocks & 4.1 & 4.1 & 4.4 & 4.9 & 5.3 & 5.0 \\
\hline
\end{tabular}

\section{Public Debt Sustainability}

\section{Kenya's public debt shows some vulnerability to growth shocks and potentially large contingent liabilities also pose additional risks to the sustainability of public debt.}

- $\quad$ Under the baseline scenario, the NPV of total public debt-to-GDP, at 42 percent in 2010, increases and peaks at 43 percent in 2012 and gradually trends down to 40 percent of GDP by 2015. Afterwards, it trends down to around 26 percent (Figure 2 and Table 1a). Given Kenya's relatively strong revenue performance, the NPV of debt-to-revenue ratio declines to below 150 percent after 2015. The debt service-to-revenue ratio falls to 22 percent by 2015, from 25 in 2009 . It declines to below 20 percent by 2030 .

- $\quad$ Alternative scenarios and stress tests indicate that Kenya's debt indicators are vulnerable to slower growth, unchanged primary balance, and materialization of contingent liabilities (see Figure 2 and Table 1b). A scenario assuming that 10 percent of 2010 GDP in potential domestic currency liabilities as of end-2010 would be paid by the government in equal tranches over a 10 -year period shows that debt indicators deteriorate notably compared with the baseline. An alternative scenario shows that a two-year growth shock leads to a rise in the NPV of debt-to-GDP ratio to 55 percent by 2014, an NPV of debt-to-revenue ratio to over 200 percent by 2020, and a rise in the debt service-to-revenue ratio to over 31 percent by 2030. Also, the scenario of permanently lower growth - baseline minus half a percentage point - results in debt indicators that are considerably higher in the long-term (e.g., by the end of the forecast period, the NPV of debt-to-GDP ratio would be 50 percent). This result reinforces the importance of implementing fiscal consolidation and expanding productive capacity in the medium term, in addition to pursuing a prudent borrowing approach, to avoid a rising debt burden. 


\section{Conclusions}

10. Kenya faces a low risk of external debt distress, reflecting the limited reliance on external borrowing and an expected improvement in macroeconomic performance. All external public debt indicators remain below the relevant country-specific debt burden thresholds. Further, although standard stress tests reveal a worsening in debt indicators, they do not result in a breach of the thresholds during the projection period.

11. Total public debt, however shows greater risk of unfavorable debt developments, especially under a shock to GDP growth, unchanged fiscal policy, or materialization of some contingent liabilities. Even temporarily lower GDP growth would set the NPV of public debt-to-GDP, the NPV of debt-to-revenue, and the ratio of debt service-to-revenue on a sharply increasing trend. A permanently unchanged primary balance from its 2010 level worsens debt dynamics notably. Potentially large but unreported contingent liabilities also pose additional risks to the sustainability of public debt.

12. The sustainability of Kenya's debt depends on macroeconomic performance and a prudent borrowing strategy. The projected investment in infrastructure and the assumed improvement in the investment climate would be crucial to sustaining strong exports and GDP growth. Additionally, Kenya's success in avoiding unsustainable debt to date reflects good management, but also limited willingness on the part of creditors to provide financing, at times due to governance concerns.

\section{The authorities were involved in the DSA exercise and concur with its}

conclusions. The staffs encourage Kenyan authorities to build on their recent medium-term debt strategy and to use tools such as the joint IMF/WB DSA template to help maintain a prudent borrowing strategy. Such a strategy should continue to consider the total concessionality and interest costs of Kenya's borrowing, maturity structure, and steps that would help guard against volatility, whether due to shocks such as droughts or to fluctuations in external assistance. Strategies to guard against shocks could include a build-up in international reserves as envisaged in the ECF framework. 
Figure 1. Kenya: Indicators of Public and Publicly Guaranteed External Debt under Alternatives Scenarios, 2010-30 1/



Rate of Debt Accumulation

- Grant-equivalent financing (\% of GDP)

Grant element of new borrowing (\% right scale)
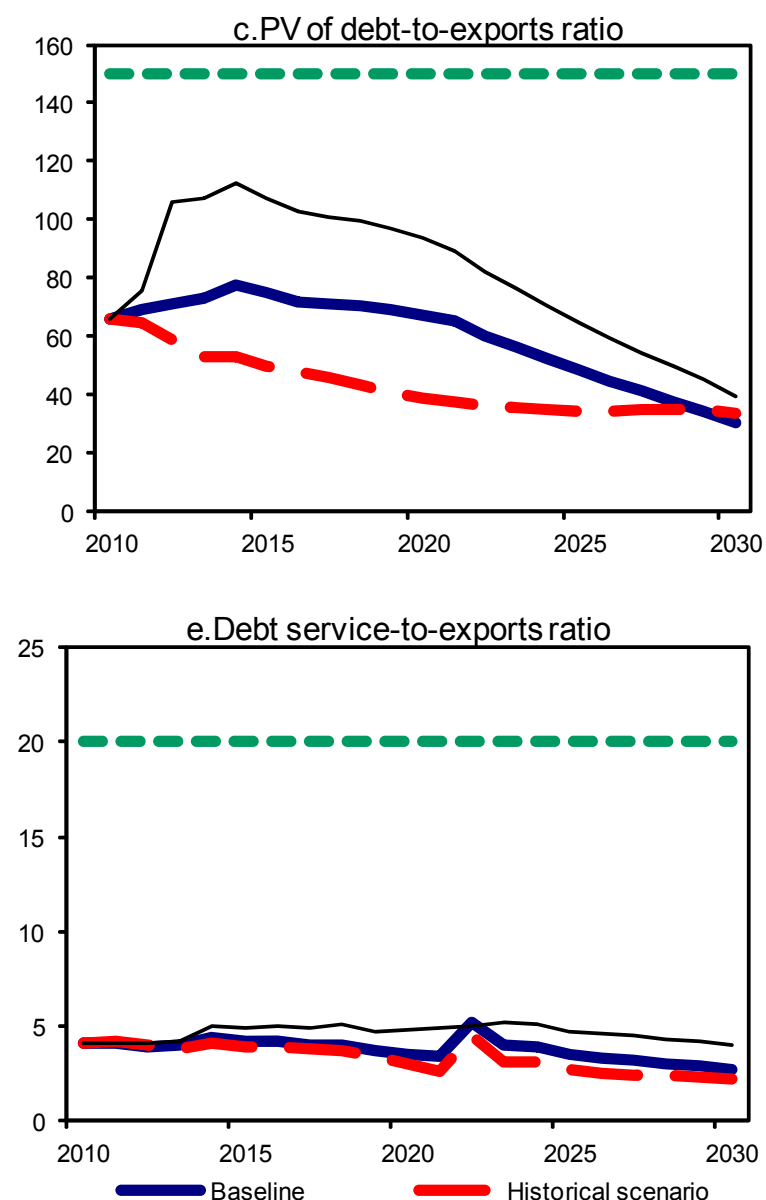

Sources: Country authorities; and staff estimates and projections
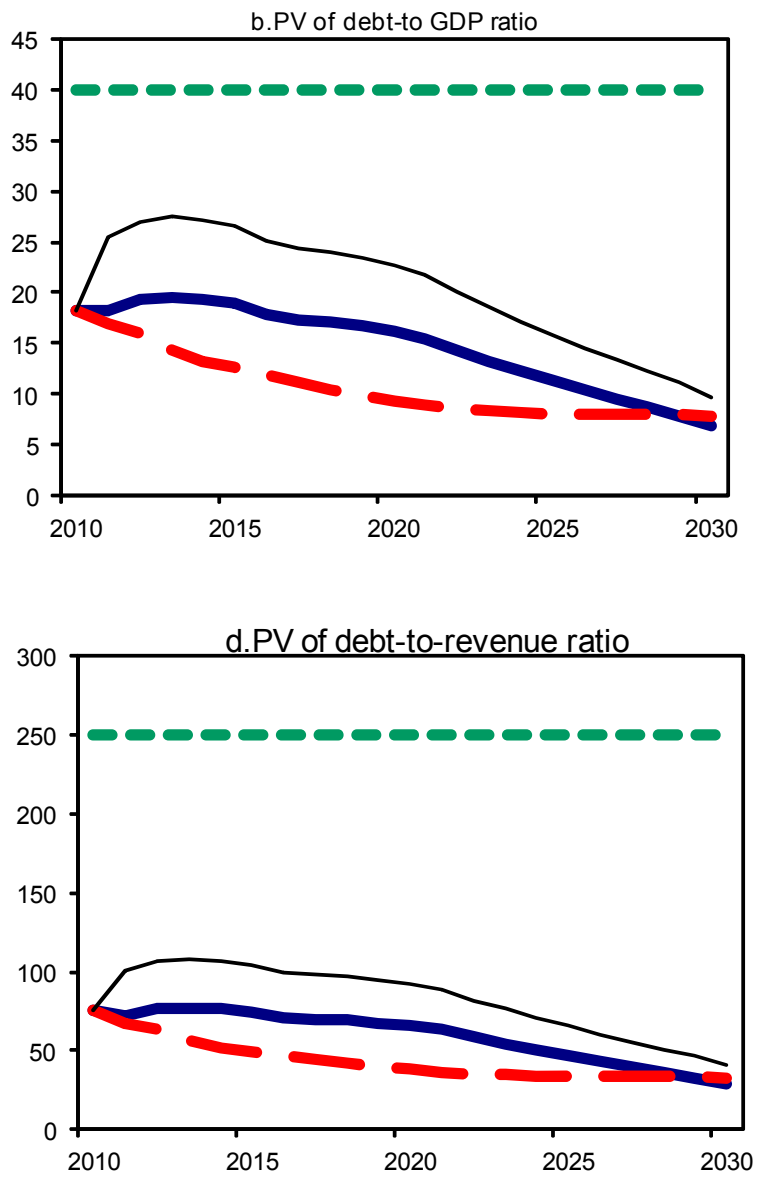

f.Debt service-to-revenue ratio

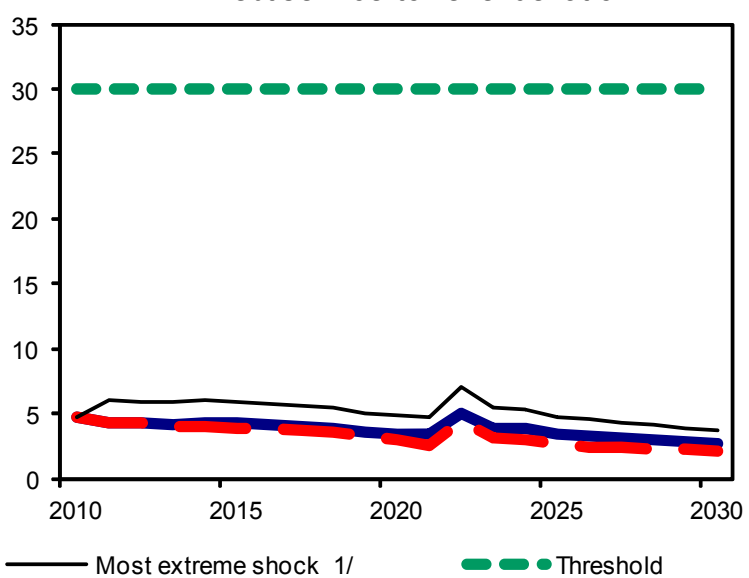

1/ The most extreme stress test is the test that yields the highest ratio in 2020. In figure b. it corresponds to a One-time depreciation shock; in c. to a Exports shock; in d. to a One-time depreciation shock; in e. to a debt service shock and in figure f. to a One-time depreciation shock. 
Figure 2.Kenya: Indicators of Public Debt Under Alternative Scenarios, 2010-30 1/
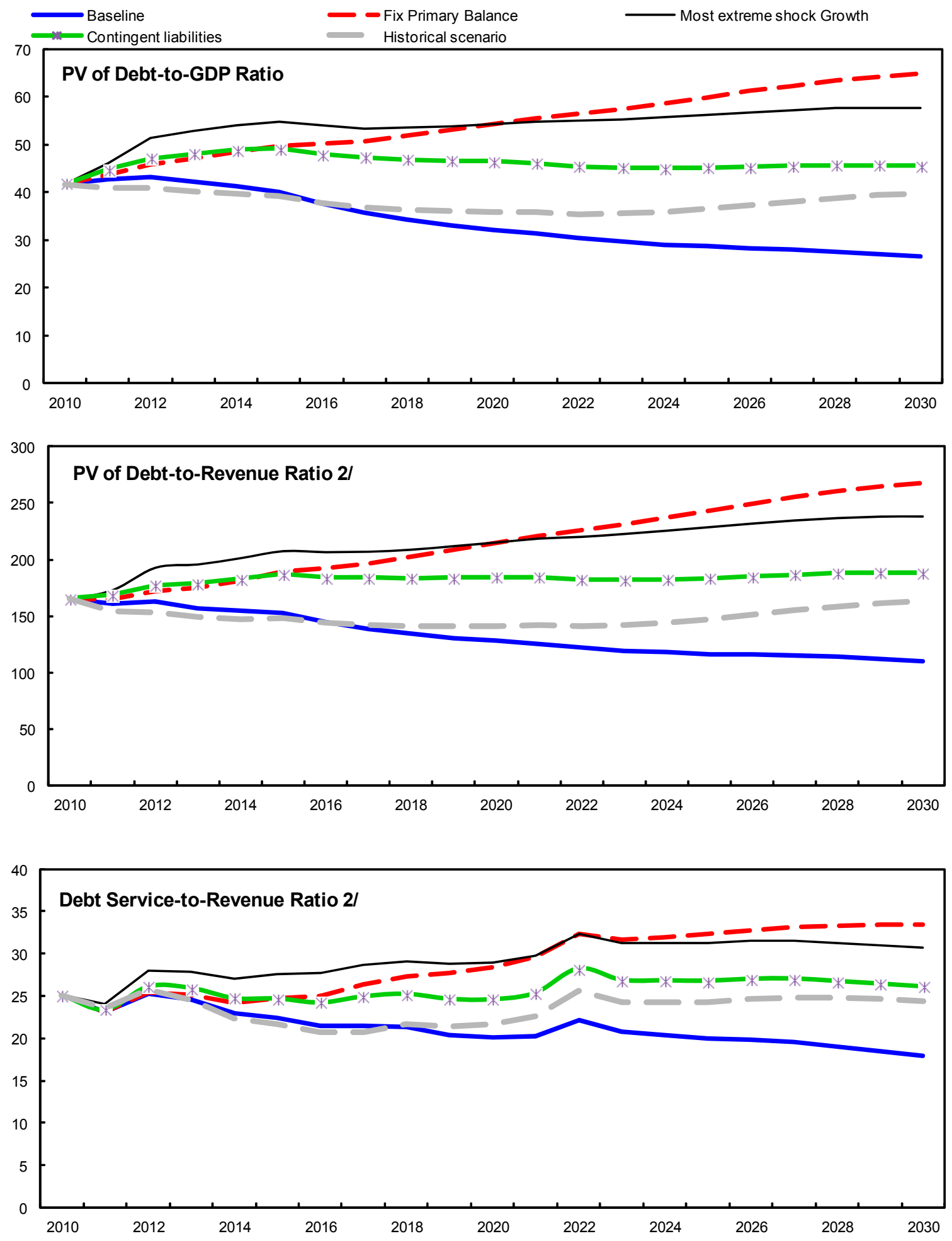

Sources: Country authorities; and staff estimates and projections.

$1 /$ The most extreme stress test is the test that yields the highest ratio in 2020.

$2 /$ Revenues are defined inclusive of grants. 
Table 1a.Kenya: Public Sector Debt Sustainability Framework, Baseline Scenario, 2007-30 (In percent of GDP, unless otherwise indicated)

\begin{tabular}{|c|c|c|c|c|c|c|c|c|c|c|c|c|c|c|c|}
\hline & \multicolumn{3}{|c|}{ Actual } & \multirow[b]{2}{*}{ Average } & \multirow[b]{2}{*}{$\begin{array}{l}\text { Standard } \\
\text { Deviation }\end{array}$} & \multirow{2}{*}{$\begin{array}{c}\text { Estimate } \\
2010 \\
\end{array}$} & \multicolumn{9}{|c|}{ Projections } \\
\hline & 2007 & 2008 & 2009 & & & & 2011 & 2012 & 2013 & 2014 & 2015 & $\begin{array}{l}2010-15 \\
\text { Average } \\
\end{array}$ & 2020 & 2030 & $\begin{array}{l}2016-30 \\
\text { Average } \\
\end{array}$ \\
\hline Public sector debt $1 /$ & 34.6 & 41.3 & 44.4 & & & 47.6 & 49.0 & 49.1 & 48.1 & 47.0 & 45.4 & & 36.9 & 28.8 & \\
\hline $\mathrm{o} / \mathrm{w}$ foreign-currency denominated & 21.1 & 23.7 & 23.8 & & & 24.2 & 24.4 & 25.2 & 25.5 & 25.1 & 24.3 & & 20.9 & 9.2 & \\
\hline Change in public sector debt & -3.5 & 6.7 & 3.1 & & & 3.2 & 1.4 & 0.1 & -1.0 & -1.1 & -1.6 & & -1.1 & -0.6 & \\
\hline Identified debt-creating flows & -5.6 & 2.5 & 1.0 & & & 2.5 & 0.1 & -1.2 & -1.4 & -1.6 & -2.1 & & -1.5 & -1.2 & \\
\hline Primary deficit & 0.5 & 2.0 & 3.1 & -0.1 & 1.7 & 3.8 & 3.2 & 2.3 & 1.6 & 1.3 & 0.8 & 2.2 & 0.5 & 0.1 & 0.3 \\
\hline Revenue and grants & 23.1 & 23.2 & 23.7 & & & 25.2 & 26.6 & 26.5 & 26.8 & 26.8 & 26.3 & & 25.2 & 24.2 & \\
\hline of which: grants & 1.1 & 1.1 & 0.9 & & & 1.0 & 1.2 & 1.3 & 1.3 & 1.3 & 0.9 & & 0.6 & 0.2 & \\
\hline Primary (noninterest) expenditure & 23.6 & 25.3 & 26.7 & & & 29.0 & 29.8 & 28.9 & 28.4 & 28.1 & 27.1 & & 25.7 & 24.3 & \\
\hline Automatic debt dynamics & -4.4 & 2.2 & -2.1 & & & -1.3 & -3.1 & -3.6 & -2.9 & -2.9 & -2.9 & & -2.0 & -1.3 & \\
\hline Contribution from interest rate/growth differential & -1.8 & -0.5 & -0.3 & & & -1.3 & -2.1 & -2.5 & -2.2 & -2.2 & -2.2 & & -1.4 & -1.0 & \\
\hline of which: contribution from average real interest rate & 0.6 & 0.0 & 0.8 & & & 0.8 & 0.5 & 0.6 & 0.9 & 0.9 & 0.8 & & 0.8 & 0.7 & \\
\hline of which: contribution from real GDP growth & -2.5 & -0.5 & -1.0 & & & -2.1 & -2.6 & -3.0 & -3.1 & -3.1 & -2.9 & & -2.2 & -1.7 & \\
\hline Contribution from real exchange rate depreciation & -2.6 & 2.7 & -1.8 & & & 0.0 & -1.0 & -1.1 & -0.7 & -0.7 & -0.7 & & $\ldots$ & $\ldots$ & \\
\hline Other identified debt-creating flows & -1.6 & -1.8 & 0.1 & & & 0.0 & 0.0 & 0.0 & 0.0 & 0.0 & 0.0 & & 0.0 & 0.0 & \\
\hline Privatization receipts (negative) & -2.2 & -1.8 & 0.0 & & & 0.0 & 0.0 & 0.0 & 0.0 & 0.0 & 0.0 & & 0.0 & 0.0 & \\
\hline Recognition of implicit or contingent liabilities & 0.0 & 0.0 & 0.0 & & & 0.0 & 0.0 & 0.0 & 0.0 & 0.0 & 0.0 & & 0.0 & 0.0 & \\
\hline Debt relief (HIPC and other) & 0.0 & 0.0 & 0.0 & & & 0.0 & 0.0 & 0.0 & 0.0 & 0.0 & 0.0 & & 0.0 & 0.0 & \\
\hline Other (specify, e.g. bank recapitalization) & 0.6 & 0.1 & 0.1 & & & 0.0 & 0.0 & 0.0 & 0.0 & 0.0 & 0.0 & & 0.0 & 0.0 & \\
\hline Residual, including asset changes & 2.1 & 4.2 & 2.1 & & & 0.7 & 1.2 & 1.3 & 0.4 & 0.5 & 0.5 & & 0.4 & 0.6 & \\
\hline \multicolumn{16}{|l|}{ Other Sustainability Indicators } \\
\hline PV of public sector debt & 13.5 & 17.7 & 38.2 & & & 41.7 & 42.7 & 43.1 & 42.2 & 41.4 & 40.0 & & 32.2 & 26.5 & \\
\hline $\mathrm{o} / \mathrm{w}$ foreign-currency denominated & 0.0 & 0.0 & 17.6 & & & 18.2 & 18.2 & 19.3 & 19.6 & 19.4 & 18.9 & & 16.2 & 6.9 & \\
\hline o/w external & ... & $\ldots$ & 17.6 & & & 18.2 & 18.2 & 19.3 & 19.6 & 19.4 & 18.9 & & 16.2 & 6.9 & \\
\hline$P V$ of contingent liabilities (not included in public sector debt) & $\ldots$ & $\ldots$ & $\ldots$ & & & & $\ldots$ & $\ldots$ & $\ldots$ & $\ldots$ & $\ldots$ & & $\ldots$ & $\ldots$ & \\
\hline Gross financing need $2 /$ & 10.7 & 12.2 & 14.7 & & & 15.9 & 15.7 & 15.6 & 14.6 & 13.6 & 12.7 & & 10.3 & 9.8 & \\
\hline $\mathrm{PV}$ of public sector debt-to-revenue and grants ratio (in percent) & 58.3 & 76.0 & 161.5 & & & 165.0 & 160.8 & 162.5 & 157.1 & 154.6 & 152.3 & & 127.8 & 109.6 & \\
\hline PV of public sector debt-to-revenue ratio (in percent) & 61.3 & 79.7 & 167.8 & & & 172.1 & 168.5 & 170.5 & 165.2 & 162.4 & 157.6 & & 130.9 & 110.7 & \\
\hline o/w external 3/ & & & 77.4 & & & 75.2 & 71.6 & 76.2 & 76.8 & 76.4 & 74.5 & & 65.7 & 29.0 & \\
\hline Debt service-to-revenue and grants ratio (in percent) $4 /$ & 26.9 & 26.4 & 27.2 & & & 25.1 & 23.1 & 25.3 & 24.6 & 22.9 & 22.4 & & 20.0 & 17.9 & \\
\hline Debt service-to-revenue ratio (in percent) $4 /$ & 28.3 & 27.7 & 28.3 & & & 26.2 & 24.3 & 26.6 & 25.9 & 24.1 & 23.2 & & 20.5 & 18.0 & \\
\hline Primary deficit that stabilizes the debt-to-GDP ratio & 4.0 & -4.7 & 0.0 & & & 0.6 & 1.8 & 2.3 & 2.5 & 2.4 & 2.4 & & 1.6 & 0.7 & \\
\hline \multicolumn{16}{|l|}{ Key macroeconomic and fiscal assumptions } \\
\hline Real GDP growth (in percent) & 7.0 & 1.6 & 2.6 & 3.7 & 2.4 & 5.0 & 5.7 & 6.5 & 6.8 & 6.8 & 6.7 & 6.3 & 6.1 & 6.1 & 6.1 \\
\hline Average nominal interest rate on forex debt (in percent) & 1.5 & 1.4 & 1.3 & 1.7 & 0.8 & 1.2 & 1.1 & 1.2 & 1.6 & 1.7 & 1.8 & 1.5 & 2.1 & 1.9 & 2.0 \\
\hline Average real interest rate on domestic debt (in percent) & 7.3 & 1.1 & 4.0 & 7.4 & 5.6 & 3.8 & 2.5 & 2.5 & 4.1 & 4.2 & 3.7 & 3.4 & 5.1 & 3.9 & 4.4 \\
\hline Real exchange rate depreciation (in percent, + indicates depreciation) & -11.7 & 13.3 & -7.7 & -4.5 & 8.0 & 0.2 & $\ldots$ & $\ldots$ & $\ldots$ & $\ldots$ & $\ldots$ & $\ldots$ & ... & ... & $\ldots$ \\
\hline Inflation rate (GDP deffator, in percent) & 5.3 & 11.9 & 6.7 & 5.9 & 3.1 & 6.6 & 7.1 & 6.5 & 4.8 & 4.9 & 5.0 & 5.8 & 5.0 & 5.0 & 5.1 \\
\hline Growth of real primary spending (deflated by GDP deflator, in percent) & 0.1 & 0.1 & 0.1 & 0.1 & 0.0 & 0.1 & 0.1 & 0.0 & 0.1 & 0.1 & 0.0 & 0.1 & 0.1 & 0.1 & 0.1 \\
\hline Grant element of new external borrowing (in percent) & $\ldots$ & ... & $\ldots$ & .. & .. & 31.4 & 35.1 & 16.9 & 25.2 & 21.1 & 23.3 & 25.5 & 27.2 & 23.4 & . \\
\hline
\end{tabular}

Sources: Country authorities; and staff estimates and projections.

$1 /$ Public debt refers to net debt of the central government and parastatals.

$2 /$ Gross financing need is defined as the primary deficit plus debt service plus the stock of short-term debt at the end of the last period.

$3 /$ Revenues excluding grants.

(the sum of interest and amortization of medium and long-term debt.

$5 /$ Historical averages and standard deviations are generally derived over the past 10 years, subject to data availability. 


\section{Table 1b. Kenya: Sensitivity Analysis for Key Indicators of Public Debt 2010-30}

\begin{tabular}{|c|c|c|c|c|c|c|c|c|}
\hline & \multicolumn{8}{|c|}{ Projections } \\
\hline & 2010 & 2011 & 2012 & 2013 & 2014 & 2015 & 2020 & 2030 \\
\hline \multicolumn{9}{|c|}{ PV of Debt-to-GDP Ratio } \\
\hline Baseline & 42 & 43 & 43 & 42 & 41 & 40 & 32 & 26 \\
\hline \multicolumn{9}{|l|}{ A. Alternative scenarios } \\
\hline A1. Real GDP growth and primary balance are at historical averages & 42 & 41 & 41 & 40 & 40 & 39 & 36 & 40 \\
\hline A2. Primary balance is unchanged from 2010 & 42 & 44 & 46 & 47 & 48 & 50 & 54 & 65 \\
\hline A3. Permanently lower GDP growth 1/ & 42 & 44 & 45 & 45 & 45 & 44 & 42 & 50 \\
\hline A4. Alternative Scenario: Recognition of Domestic Contingent Liabilities During 2011-20 & 42 & 45 & 47 & 48 & 49 & 49 & 47 & 45 \\
\hline \multicolumn{9}{|l|}{ B. Bound tests } \\
\hline B1. Real GDP growth is at historical average minus one standard deviations in 2011-2012 & 42 & 46 & 51 & 53 & 54 & 55 & 54 & 58 \\
\hline B2. Primary balance is at historical average minus one standard deviations in 2011-2012 & 42 & 42 & 42 & 41 & 41 & 40 & 34 & 30 \\
\hline B3. Combination of B1-B2 using one half standard deviation shocks & 42 & 42 & 43 & 44 & 46 & 46 & 46 & 49 \\
\hline B4. One-time 30 percent real depreciation in 2011 & 42 & 50 & 50 & 48 & 47 & 46 & 37 & 32 \\
\hline B5. 10 percent of GDP increase in other debt-creating flows in 2011 & 42 & 52 & 52 & 51 & 50 & 49 & 40 & 33 \\
\hline \multicolumn{9}{|c|}{ PV of Debt-to-Revenue Ratio 2/ } \\
\hline Baseline & 165 & 161 & 162 & 157 & 155 & 152 & 128 & 110 \\
\hline \multicolumn{9}{|l|}{ A. Alternative scenarios } \\
\hline A1. Real GDP growth and primary balance are at historical averages & 165 & 154 & 153 & 149 & 147 & 148 & 141 & 163 \\
\hline A2. Primary balance is unchanged from 2010 & 165 & 164 & 172 & 175 & 181 & 189 & 215 & 268 \\
\hline A3. Permanently lower GDP growth 1/ & 165 & 164 & 168 & 166 & 167 & 168 & 166 & 207 \\
\hline A4. Alternative Scenario: Recognition of Domestic Contingent Liabilities During 2011-20 & 166 & 169 & 177 & 179 & 183 & 187 & 185 & 188 \\
\hline \multicolumn{9}{|l|}{ B. Bound tests } \\
\hline B1. Real GDP growth is at historical average minus one standard deviations in 2011-2012 & 165 & 173 & 193 & 196 & 201 & 207 & 215 & 238 \\
\hline B2. Primary balance is at historical average minus one standard deviations in 2011-2012 & 165 & 157 & 158 & 154 & 153 & 152 & 134 & 123 \\
\hline B3. Combination of B1-B2 using one half standard deviation shocks & 165 & 159 & 162 & 165 & 170 & 175 & 181 & 201 \\
\hline B4. One-time 30 percent real depreciation in 2011 & 165 & 188 & 188 & 180 & 177 & 174 & 149 & 133 \\
\hline B5. 10 percent of GDP increase in other debt-creating flows in 2011 & 165 & 196 & 197 & 190 & 187 & 185 & 157 & 135 \\
\hline \multicolumn{9}{|c|}{ Debt Service-to-Revenue Ratio 2/ } \\
\hline Baseline & 25 & 23 & 25 & 25 & 23 & 22 & 20 & 18 \\
\hline \multicolumn{9}{|l|}{ A. Alternative scenarios } \\
\hline A1. Real GDP growth and primary balance are at historical averages & 25 & 24 & 26 & 24 & 22 & 22 & 22 & 24 \\
\hline A2. Primary balance is unchanged from 2010 & 25 & 23 & 25 & 25 & 24 & 25 & 28 & 33 \\
\hline A3. Permanently lower GDP growth 1/ & 25 & 23 & 26 & 25 & 24 & 23 & 23 & 27 \\
\hline A4. Alternative Scenario: Recognition of Domestic Contingent Liabilities During 2011-20 & 25 & 23 & 26 & 26 & 25 & 25 & 25 & 26 \\
\hline \multicolumn{9}{|l|}{ B. Bound tests } \\
\hline B1. Real GDP growth is at historical average minus one standard deviations in 2011-2012 & 25 & 24 & 28 & 28 & 27 & 28 & 29 & 31 \\
\hline B2. Primary balance is at historical average minus one standard deviations in 2011-2012 & 25 & 23 & 25 & 24 & 22 & 21 & 20 & 18 \\
\hline B3. Combination of B1-B2 using one half standard deviation shocks & 25 & 24 & 26 & 25 & 23 & 23 & 25 & 26 \\
\hline B4. One-time 30 percent real depreciation in 2011 & 25 & 24 & 27 & 27 & 25 & 25 & 23 & 21 \\
\hline B5. 10 percent of GDP increase in other debt-creating flows in 2011 & 25 & 23 & 28 & 29 & 27 & 26 & 22 & 19 \\
\hline
\end{tabular}

Sources: Country authorities; and staff estimates and projections.

1/ Assumes that real GDP growth is at baseline minus one standard deviation divided by the square root of the length of the projection period. 2/ Revenues are defined inclusive of grants. 
Table 2a. Kenya: External Debt Sustalnabllity Framework, Baselline Scenarlo, 2007-30 1/

\begin{tabular}{|c|c|c|c|c|c|c|c|c|c|c|c|c|c|c|c|}
\hline & \multicolumn{3}{|c|}{ Actual } & \multirow{2}{*}{$\begin{array}{l}\text { Historical } \\
\text { Average }\end{array}$} & \multirow{2}{*}{$\begin{array}{l}\text { Standard } \\
\text { Devation }\end{array}$} & \multicolumn{6}{|c|}{ Projections } & \multicolumn{3}{|l|}{$2010-15$} & \multirow{2}{*}{$\begin{array}{l}2016-30 \\
\text { Average }\end{array}$} \\
\hline & 2007 & 2008 & 2009 & & & 2010 & 2011 & 2012 & 2013 & 2011 & 2016 & Awerage & 2020 & 2030 & \\
\hline External debt (nom inal) 1/ & 23.0 & 25.4 & 24.9 & & & 25.1 & 24.9 & 26.0 & 27.2 & 26.9 & 25.8 & & 21.7 & 9.2 & \\
\hline oiw pub lic and pub licly guaranteed (PPG) & 21.1 & 23.7 & 23.8 & & & 24.2 & 24.4 & 25.2 & 25.5 & 25.1 & 24.3 & & 20.9 & 9.2 & \\
\hline Change in external debt & -2.9 & 2.4 & -0.5 & & & 0.2 & -0.2 & 1.2 & 1.1 & -0.3 & -1.1 & & -0.8 & -1.0 & \\
\hline Identified net debt-creating fows & -4.1 & 2.3 & 4.8 & & & 5.2 & 4.5 & 2.9 & 2.2 & 1.6 & 0.6 & & -0.8 & -4.3 & \\
\hline Non-interestcurrent account deficit & 3.5 & 6.3 & 5.4 & 1.8 & 2.6 & 7.3 & 7.2 & 5.8 & 5.1 & 4.5 & 2.9 & & 1.1 & -3.3 & -0.1 \\
\hline De ficit in balance of goods and services & 11.1 & 14.2 & 13.1 & & & 13.8 & 13.6 & 12.6 & 12.7 & 12.5 & 11.6 & & 8.9 & 2.9 & \\
\hline Exports & 26.0 & 27.6 & 25.2 & & & 27.6 & 26.2 & 27.2 & 26.8 & 25.0 & 25.3 & & 23.9 & 23.1 & \\
\hline Imports & 37.1 & 41.8 & 38.3 & & & 41.3 & 39.8 & 39.8 & 39.4 & 37.5 & 36.9 & & 32.8 & 26.0 & \\
\hline Net currest thanafere (ne gative = in flow) & -77 & -77 & -7 \% & \& 9 & 10 & -83 & -6? & - ด & $-7 ?$ & -7 6 & -79 & & $-7 ?$ & -6 & - 88 \\
\hline o/w official & -0.1 & 0.1 & 0.1 & & & 0.1 & 0.1 & 0.1 & 0.0 & 0.0 & 0.0 & & 0.0 & 0.0 & \\
\hline Other curent account flows (negative = net in flow) & 0.1 & -0.2 & -0.1 & & & -0.1 & -0.1 & -0.3 & -0.4 & -0.5 & -0.7 & & -0.7 & -0.2 & \\
\hline Net FDI (ne gative $=$ inflow) & -3.6 & -2.2 & -1.5 & $-1 / 4$ & 1.0 & -1.3 & -1.8 & -1.7 & -1.7 & -1.6 & -1.2 & & -1.1 & -0.6 & -1.0 \\
\hline Endogenous debt dynamic 52 f & -4.0 & -1.8 & 0.9 & & & -0.8 & -1.0 & -1.1 & -1.2 & -1.3 & -1.2 & & -0.8 & -0.4 & \\
\hline Contribution from nom inal interest rate & 0.1 & 0.1 & 0.1 & & & 0.3 & 0.3 & 0.3 & 0.1 & 0.1 & 0.1 & & 0.1 & 0.2 & \\
\hline Contribution from real GDP growth & -1.5 & -0.3 & -0.7 & & & -1.1 & -1.3 & -1.4 & -1.6 & -1.7 & -1.6 & & -1.2 & -0.6 & \\
\hline Contribution from price and exchange rate changes & -2.9 & -1.9 & 1.2 & & & & & & & & & & & & \\
\hline Re sidual (3-4) $3 /$ & 1.2 & 0.1 & -5.3 & & & -5.0 & -4.6 & -1.8 & -1.1 & -1.9 & -1.6 & & 0.0 & 3.3 & \\
\hline o/w exceptional financing & 0.0 & 0.0 & 0.0 & & & 0.0 & 0.0 & 0.0 & 0.0 & 0.0 & 0.0 & & 0.0 & 0.0 & \\
\hline PV of external debt $4 /$ & & ... & 18.7 & & & 19 & 18.6 & 20.1 & 21.2 & 212 & 20.5 & & 17.0 & 6.9 & \\
\hline In percent of exports & & & 74.0 & & & 69 & 71 & 74 & 79.3 & 85.1 & 80.7 & & 70.9 & 30.1 & \\
\hline PV of PPG external debt & ... & ... & 17.6 & & & 18.2 & 18.2 & 19.3 & 19.6 & 19.4 & 18.9 & & 16.2 & 6.9 & \\
\hline In percent of exports & $\ldots$ & ... & 69.9 & & & 66.0 & 69.2 & 70.9 & 73.2 & 77.8 & 74.7 & & 67.5 & 30.1 & \\
\hline In persent of gnuernment revanues & $\cdots$ & $\cdots$ & 774 & & & $75 ?$ & $71 \mathrm{f}$ & $76 ?$ & 768 & 764 & 745 & & 6.57 & 290 & \\
\hline Debt service-to-exports ratio (in percent) & 6.4 & 5.2 & 5.4 & & & 5.1 & 5.5 & 4.6 & 4.6 & 4.9 & 4.6 & & 3.5 & 2.7 & \\
\hline PPG debt sevice-to-exports ratio (in percent) & 4.8 & 4.0 & 4.0 & & & 4.1 & 4.1 & 3.9 & 4.0 & 4.4 & 4.2 & & 3.5 & 2.7 & \\
\hline PPG debt se vice-to-revenue ratio (in percent) & 5.6 & 4.9 & 4.4 & & & 4.7 & 4.3 & 4.2 & 4.2 & 4.3 & 4.2 & & 3.4 & 2.6 & \\
\hline Total gros s financing need (Eillions of U.S. dollars) & 0.4 & 1.7 & 1.5 & & & 2.4 & 2.5 & 2.1 & 2.1 & 2.1 & 1.7 & & 0.8 & -9.7 & \\
\hline Non-interest current sccount $t$ e ficit that stabilices debt ratic & 6.4 & 3.0 & 5.0 & & & 7.1 & 7.4 & 4.8 & 4.0 & 4.0 & 4.0 & & 1.0 & -2.2 & \\
\hline \multicolumn{16}{|l|}{ Key macroeconomic as sumptions } \\
\hline Real GDP growth (in percent & 7.0 & 1.6 & 2.6 & 3.7 & 2.4 & 5.0 & 5.7 & 6.5 & 6.8 & 6.8 & 6.7 & 6.3 & 6.1 & 6.1 & 6.1 \\
\hline GDP de flat tor in US dollar terms (change in percent) & 12.8 & 8.9 & -4.6 & 6.1 & 6.2 & 4.0 & 5.0 & 6.4 & 4.8 & 4.9 & 5.0 & 5.0 & 5.0 & 5.0 & 5.1 \\
\hline E ffe otive interest rate (peroent) $6 /$ & 2.0 & 1.8 & 1.1 & 1.8 & 0.8 & 1.1 & 1.3 & 1.3 & 1.6 & 1.6 & 1.7 & 1.6 & 2.0 & 1.8 & \\
\hline Grow th of exports of G\&S (US dollar terms, in percent) & 18.2 & 17.4 & -10.6 & 11.1 & 9.8 & 19.3 & 5.6 & 17.5 & 10.2 & 4.5 & 13.7 & 11.8 & 10.8 & 112 & 10.9 \\
\hline Grow th of imports of G\&S (US dollar terms, in percent) & 23.2 & 24.6 & -10.4 & 13.8 & 13.3 & 17.8 & 6.8 & 13.4 & 10.9 & 6.6 & 10.3 & 11.0 & 8.9 & 8.8 & 9.0 \\
\hline Grant elemen: of new public sector borrowing (in percent) & & & & $\cdots$ & ... & 31.4 & 35.1 & 16.9 & 25.2 & 21.1 & 23.3 & 25.5 & 27.2 & 23.4 & 25.6 \\
\hline Government re venues (excluding grants, in percent of GDP) & 22.0 & 22.1 & $22 . \ddot{8}$ & & $\cdots$ & 24.2 & 25.4 & 25.3 & 25.5 & 25.5 & 25.4 & & 24.8 & 23.9 & 24.4 \\
\hline Aid flows (in Eillions of US dellars) 7/ & 0.3 & 0.7 & 0.3 & & & 0.7 & 1.1 & 1.1 & 1.3 & $12 \frac{2}{7}$ & 1.0 & & 1.4 & 1.3 & \\
\hline $\begin{array}{l}\text { O/w Grants } \\
\text { O/w Conces sional loans }\end{array}$ & $\begin{array}{l}0.3 \\
0.0\end{array}$ & $\begin{array}{l}0.3 \\
0.4\end{array}$ & $\begin{array}{l}0.3 \\
0.0\end{array}$ & & & $\begin{array}{l}0.3 \\
0.3\end{array}$ & $\begin{array}{l}0.4 \\
0.7\end{array}$ & $\begin{array}{l}0.5 \\
0.6\end{array}$ & $\begin{array}{l}0.6 \\
0.7\end{array}$ & $\begin{array}{l}0.7 \\
0.5\end{array}$ & $\begin{array}{l}0.5 \\
0.5\end{array}$ & & $\begin{array}{l}0.3 \\
0.8\end{array}$ & $\begin{array}{l}0.7 \\
0.8\end{array}$ & \\
\hline Grantequivalent financing (in percent of GDP) \&/ & $\ldots$ & 0.4 & ... & & & 1.5 & 2.5 & 2.0 & 2.2 & 1.9 & 1.5 & & 1.1 & 0.4 & 0.9 \\
\hline Grantequivalent financing (in percent of external financing) 8/ & & $\ldots$ & $\ldots$ & & & 50.5 & 51.2 & 35.0 & 44.7 & 44.8 & 42.8 & & 44.7 & 47.5 & 45.4 \\
\hline \multicolumn{16}{|l|}{ Mem oranoum nems: } \\
\hline Nominal GDP (Billions of US dollars) & 272 & 30.0 & 29.4 & & & 32.1 & 35.6 & 40.3 & 45.2 & 50.6 & 56.7 & & 100.0 & 293.7 & \\
\hline Nominal dollar GDP growth & 20.7 & 10.5 & -2.1 & & & 9.2 & 11.0 & 13.3 & 12.0 & 12.1 & 12.0 & 11.6 & 11.4 & 11.4 & 11.6 \\
\hline PV of PPG external debt (in Billions of US dollars) & & & 5.3 & & & 5.8 & 6.5 & 7.8 & 8.9 & 9.8 & 10.7 & & 16.2 & 20.4 & \\
\hline (PVt-PVt-1) GDPt-1 (in percent) & & & & & & 1.6 & 2.1 & 3.7 & 2.7 & 2.2 & 1.8 & 2.4 & 1.3 & -0.2 & 0.7 \\
\hline Gross workers' remittances (Billions of US dollars) & 0.9 & 1.0 & 1.0 & & & 1.0 & 1.1 & 1.3 & 1.6 & 1.9 & 2.2 & & 3.5 & 8.4 & \\
\hline PV of PPG external debt (in percent of GDF + remittances) & & $\ldots$ & 17.1 & & & 17.6 & 17.6 & 18.7 & 18.9 & 18.8 & 18.2 & & 15.3 & 6.8 & \\
\hline PV of PPG esternal debt (in peroent of exports + remittancos) & & ... & 61.8 & & & 59.3 & 61.9 & 63.1 & 34.7 & 67.9 & 64.9 & & 59.0 & 26.8 & \\
\hline Debt service of PPG external debt (in percent of exports + remittances) & $\ldots$ & $\ldots$ & 3.5 & & & 3.7 & 3.7 & 3.5 & 3.5 & 3.9 & 3.7 & & 3.1 & 2.4 & \\
\hline
\end{tabular}

Sources: Countryauthoribes; and staffestmates and prokections.

$1 /$ Includes both public and private sector external debt

2/ Derived as $[r-g-p(1+g))(1+g+p+g p)$ times previous period debt ratio, with $r=$ nominal interest rate; $g=$ real GDP growth rate, and $p=$ growth rate of GDP de flator in U.S. dollar terms.

$3 /$ Includes exceptional financing (i.e., changes in arrears and debt reliefi; changes in gross foreign assets; and valuation adjustments. For projections also includes contibution fom price and exchange rate changes.

4/ Ass umes that PV of private sector debt is equivalent to it face value.

$5 /$ Current-year in terest payments divided by pre vious period debt stock.
$6 /$ His torical a verages and stand ard de viations are generallyderived ove the past 10 years, subject to data availa bility.

6/ His trical a verages and stand ard de viations are generdlof

$8 /$ Grantequivalent financing includes grants provided directy to the government and through new borrowing (differense between the face value and the PV of new debt). 
Table 2b. Kenya: Sensitivity Analysis for Key Indicators of Public and Publicly Guaranteed External Debt, 2010-30

(In percent)

\begin{tabular}{|c|c|c|c|c|c|c|c|c|}
\hline & & & & Projec & & & & \\
\hline & 2010 & 2011 & 2012 & 2013 & 2014 & 2015 & 2020 & 2030 \\
\hline PV of debt-to & & & & & & & & \\
\hline Baseline & 18.2 & 18.2 & 19.3 & 19.6 & 19.4 & 18.9 & 16.2 & 6.9 \\
\hline A. Alternative Scenarios & & & & & & & & \\
\hline A1. Key variables at their historical averages in $2010-2030$ 1/ & 18.2 & 17.0 & 16.0 & 14.3 & 13.3 & 12.6 & 9.3 & 7.8 \\
\hline A2. New public sector loans on less favorable terms in $2010-20302 /$ & 18.2 & 19.3 & 20.8 & 22.4 & 22.8 & 22.9 & 22.0 & 11.9 \\
\hline B. Bound Tests & & & & & & & & \\
\hline B1. Real GDP growth at historical average minus one standard deviation in 2011-2012 & 18.2 & 18.9 & 21.2 & 21.5 & 21.3 & 20.8 & 17.7 & 7.6 \\
\hline B2. Export value growth at historical average minus one standard deviation in 2011-2012 3/ & 18.2 & 19.0 & 23.8 & 23.8 & 23.3 & 22.5 & 18.5 & 7.5 \\
\hline B3. US dollar GDP deflator at historical average minus one standard deviation in 2011-2012 & 18.2 & 19.1 & 21.6 & 21.9 & 21.8 & 21.2 & 18.1 & 7.8 \\
\hline B4. Net non-debt creating flows at historical average minus one standard deviation in 2011-2012 4/ & 18.2 & 19.5 & 22.2 & 22.2 & 21.9 & 21.2 & 17.6 & 7.3 \\
\hline B5. Combination of B1-B4 using one-half standard deviation shocks & 18.2 & 19.8 & 26.4 & 26.4 & 25.9 & 25.0 & 20.7 & 8.4 \\
\hline B6. One-time 30 percent nominal depreciation relative to the baseline in 20115 / & 18.2 & 25.4 & 27.0 & 27.4 & 27.2 & 26.5 & 22.6 & 9.7 \\
\hline
\end{tabular}

PV of debt-to-exports ratio

Baseline

66.0

69.2

70.9

73.2

77.8

74.7

67.5

30.1

A1. Key variables at their historical averages in 2010-2030 1/

A2. New public sector loans on less favorable terms in 2010-2030 2/

\section{B. Bound Tests}

B1. Real GDP growth at historical average minus one standard deviation in 2011-2012

B2. Export value growth at historical average minus one standard deviation in 2011-2012 3/

B4. Net non-debt creating flows at historical average minus one standard deviation in 2011-2012 4/

B5. Combination of B1-B4 using one-half standard deviation shocks

B6. One-time 30 percent nominal depreciation relative to the baseline in $20115 /$

66.0

66.0

66.0

\section{B. Bound Tests}

B1. Real GDP growth at historical average minus one standard deviation in 2011-2012

B2. Export value growth at historical average minus one standard deviation in 2011-2012 3/

B3. US dollar GDP deflator at historical average minus one standard deviation in 2011-2012
B4. Net non-debt creating flows at historical average minus one standard deviation in 2011-2012 4/

B4. Net non-debt creating flows at historical average minus one standar

B6. One-time 30 percent nominal depreciation relative to the baseline in $20115 /$

$\begin{array}{rrrrrrr}74.6 & 83.6 & 84.2 & 83.8 & 81.8 & \mathbf{7 2 . 1} & 31.8 \\ 74.8 & 94.1 & 93.1 & 91.5 & 88.5 & \mathbf{7 5 . 3} & 31.4 \\ 75.1 & 85.3 & 85.9 & 85.5 & 83.4 & \mathbf{7 3 . 5} & 32.5 \\ 76.9 & 87.6 & 87.1 & 86.0 & 83.4 & \mathbf{7 1 . 7} & 30.5 \\ 78.2 & 104.4 & 103.5 & 101.8 & 98.5 & \mathbf{8 4 . 1} & 35.2 \\ 100.1 & 106.7 & 107.4 & 106.9 & 104.4 & \mathbf{9 2 . 0} & 40.6\end{array}$


Table 2b.Kenya: Sensitivity Analysis for Key Indicators of Public and Publicly Guaranteed External Debt, 2010-30 (concluded) (In percent)

\begin{tabular}{|c|c|c|c|c|c|c|c|c|}
\hline & & & & Project & & & & \\
\hline & 2010 & 2011 & 2012 & 2013 & 2014 & 2015 & 2020 & 2030 \\
\hline Debt service-to- & & & & & & & & \\
\hline Baseline & 4.1 & 4.1 & 3.9 & 4.0 & 4.4 & 4.2 & 3.5 & 2.7 \\
\hline A. Alternative Scenarios & & & & & & & & \\
\hline A1. Key variables at their historical averages in 2010-2030 1/ & 4.1 & 4.2 & 4.0 & 3.8 & 4.1 & 3.9 & 3.0 & 2.3 \\
\hline A2. New public sector loans on less favorable terms in $2010-2030$ 2/ & 4.1 & 4.1 & 4.1 & 4.2 & 5.0 & 4.9 & 4.8 & 4.0 \\
\hline B. Bound Tests & & & & & & & & \\
\hline B1. Real GDP growth at historical average minus one standard deviation in 2011-2012 & 4.1 & 4.1 & 3.9 & 4.0 & 4.4 & 4.2 & 3.5 & 2.7 \\
\hline B2. Export value growth at historical average minus one standard deviation in 2011-2012 3/ & 4.1 & 4.3 & 4.9 & 5.4 & 5.9 & 5.6 & 4.7 & 3.7 \\
\hline B3. US dollar GDP deflator at historical average minus one standard deviation in 2011-2012 & 4.1 & 4.1 & 3.9 & 4.0 & 4.4 & 4.2 & 3.5 & 2.7 \\
\hline B4. Net non-debt creating flows at historical average minus one standard deviation in 2011-2012 4/ & 4.1 & 4.1 & 4.1 & 4.3 & 4.7 & 4.5 & 3.8 & 2.9 \\
\hline B5. Combination of B1-B4 using one-half standard deviation shocks & 4.1 & 4.1 & 4.4 & 4.9 & 5.3 & 5.0 & 4.2 & 3.3 \\
\hline B6. One-time 30 percent nominal depreciation relative to the baseline in 20115 / & 4.1 & 4.1 & 3.9 & 4.0 & 4.4 & 4.2 & 3.5 & 2.7 \\
\hline Debt service-to-r & & & & & & & & \\
\hline Baseline & 4.7 & 4.3 & 4.2 & 4.2 & 4.3 & 4.2 & 3.4 & 2.6 \\
\hline A. Alternative Scenarios & & & & & & & & \\
\hline A1. Key variables at their historical averages in $2010-20301 /$ & 4.7 & 4.3 & 4.3 & 4.0 & 4.0 & 3.9 & 2.9 & 2.2 \\
\hline A2. New public sector loans on less favorable terms in $2010-2030$ 2/ & 4.7 & 4.3 & 4.4 & 4.4 & 4.9 & 4.9 & 4.7 & 3.8 \\
\hline B. Bound Tests & & & & & & & & \\
\hline B1. Real GDP growth at historical average minus one standard deviation in 2011-2012 & 4.7 & 4.5 & 4.7 & 4.6 & 4.8 & 4.6 & 3.8 & 2.9 \\
\hline B2. Export value growth at historical average minus one standard deviation in 2011-2012 3/ & 4.7 & 4.3 & 4.3 & 4.7 & 4.8 & 4.6 & 3.8 & 2.9 \\
\hline B3. US dollar GDP deflator at historical average minus one standard deviation in 2011-2012 & 4.7 & 4.5 & 4.7 & 4.7 & 4.9 & 4.7 & 3.8 & 2.9 \\
\hline 'B4. Net non-debt creating flows at historical average minus one standard deviation in 2011-2012 4/ & 4.7 & 4.3 & 4.4 & 4.5 & 4.6 & 4.5 & 3.7 & 2.8 \\
\hline B5. Combination of B1-B4 using one-half standard deviation shocks & 4.7 & 4.5 & 4.9 & 5.2 & 5.3 & 5.2 & 4.2 & 3.3 \\
\hline B6. One-time 30 percent nominal depreciation relative to the baseline in 20115 / & 4.7 & 6.0 & 5.9 & 5.9 & 6.1 & 5.9 & 4.8 & 3.7 \\
\hline Memorandum item: & & & & & & & & \\
\hline Grant element assumed on residual financing (i.e., financing required above baseline) 6/ & 20.7 & 20.7 & 20.7 & 20.7 & 20.7 & 20.7 & 20.7 & 20.7 \\
\hline
\end{tabular}

Sources: Country authorities; and staff estimates and projections.

1/ Variables include real GDP growth, growth of GDP deflator (in U.S. dollar terms), non-interest current account in percent of GDP, and non-debt creating flows.

2/ Assumes that the interest rate on new borrowing is by 2 percentage points higher than in the baseline., while grace and maturity periods are the same as in the baseline.

3/ Exports values are assumed to remain permanently at the lower level, but the current account as a share of GDP is assumed to return to its baseline level after the shock (implicitly assuming an offsetting adjustment in import levels).

4/ Includes official and private transfers and FDI.

5/ Depreciation is defined as percentage decline in dollar/local currency rate, such that it never exceeds 100 percent.

6/ Applies to all stress scenarios except for A2 (less favorable financing) in which the terms on all new financing are as specified in footnote 2. 
Press Release No. 11/22

FOR IMMEDIATE RELEASE

January 31,2011
International Monetary Fund

Washington, D.C. 20431 USA

\section{IMF Executive Board Approves Three-Year, US\$508.7 Million Arrangement Under Extended Credit Facility for Kenya}

The Executive Board of the International Monetary Fund (IMF) today approved a three-year arrangement under the Extended Credit Facility for Kenya in an amount equivalent to SDR 325.68 million (about US\$508.7 million). The program is aimed at protecting Kenya's external position, while allowing a gradual fiscal adjustment. The Executive Board's approval will immediately enable an initial disbursement of an amount equivalent to SDR 65.1 million (about US\$101.7 million).

The program is designed to help rebuild Kenya's international reserves, by supporting the conditions for sustainable growth while preserving macroeconomic stability. It will help address balance-of-payments financing needs and provide a reserve cushion to help the country deal with adverse shocks.

Following the Executive Board's discussion on Kenya, Mr. John Lipsky, First Deputy Managing Director and Acting Chair, stated:

"Kenya's economy is bouncing back from the slowdown that accompanied the global financial crisis. Fiscal stimulus has boosted the recovery, especially in construction, and benign weather conditions have supported a rebound in agriculture. The ratification of the new constitution by the August 2010 referendum has spurred confidence as it provides an opportunity to address long-standing social and institutional problems and implement additional reforms, including on fiscal decentralization, the public expenditure framework, and land ownership.

"Sustaining high growth will require addressing remaining macroeconomic vulnerabilities. The ongoing recovery will further weaken Kenya's external position and the rise in public debt resulting from the fiscal expansion should be reversed. However, fiscal consolidation needs to accommodate the costs of implementing the new constitution as well as highpriority public investments. 
“The authorities' program under the three-year ECF arrangement aims at boosting international reserves and strengthening the fiscal position. The arrangement provides a reserves cushion to reduce vulnerabilities to external shocks, including a projected deterioration of the terms of trade in the next two years. The program will help maintain fiscal discipline as fiscal decentralization gets underway, while keeping inflation low in the context of Kenya's floating exchange rate regime.

"The program targets a gradual reduction in the central government primary balance through tax reform and strict control of current spending. Tax measures include the reform of the value-added and income taxes to improve coverage and compliance. A successful implementation of constitutional provisions will also address governance issues, by strengthening the judiciary and overhauling the public expenditure management framework. The program will create the fiscal space necessary to implement the constitution, raise infrastructure investment, and protect spending aimed at poverty reduction," said Mr. Lipsky.

\section{ANNEX}

\section{$\underline{\text { Recent Economic Developments }}$}

The Kenyan economy is recovering well from several shocks: two consecutive droughts, social strife, and the global economic downturn. Despite the negative impact of these shocks, economic growth remained strong in 2010, with growth estimated at above 5 percent from 2.6 percent in 2009 , driven mainly by a rebound in agriculture and a boost in construction activity, helped in particular by the government's renewed efforts to upgrade infrastructure.

Kenya's objective is to achieve higher and more sustainable economic growth, with increased employment opportunities for youth. The Poverty Reduction Strategy Paper aims for real GDP growth of 10 percent in the medium term. Achieving this growth rate will enable Kenya to direct more resources to sectors that benefit the poor, including education and health, and help reduce poverty

\section{Program Summary}

The program is aimed at boosting international reserves while adopting a gradual fiscal adjustment over a three-year horizon. Its main objectives are : i) to raise real GDP to close to 7 percent; ii) bring the public debt-to-GDP ratio to below 45 percent over the medium term; and iii) keep inflation at 5 percent while maintaining a floating exchange rate regime.

To this end, the government will scale up investment in the key priority social and economic sectors as well as in critical infrastructures, in particular geothermal power generation to deal with climate-change challenges In addition, structural reforms will be implemented in the area of public financial management, public services, business regulation, and the financial sector. 
Kenya: Selected Economic Indicators, 2008/09-2013/2014 1/

\begin{tabular}{|c|c|c|c|c|c|c|}
\hline & \multirow{2}{*}{$\begin{array}{r}\text { Actual } \\
2008 / 09 \\
\end{array}$} & \multirow{2}{*}{$\begin{array}{r}\text { Estimate } \\
2009 / 10 \\
\end{array}$} & \multicolumn{4}{|c|}{ Projections } \\
\hline & & & $2010 / 11$ & $2011 / 12$ & $2012 / 13$ & $2013 / 14$ \\
\hline \multicolumn{7}{|l|}{ National accounts and prices } \\
\hline Nominal GDP (market prices, in billions of Kenya shillings) & 2,176 & 2,410 & 2,713 & 3,075 & 3,464 & 3,880 \\
\hline Real GDP growth (market prices) & 2.1 & 3.8 & 5.4 & 6.1 & 6.7 & 6.8 \\
\hline GDP deflator (average) 2/ & 9.3 & 6.7 & 6.9 & 6.8 & 5.6 & 4.9 \\
\hline Consumer price index (annual average) $2 /$ & 15.0 & 5.3 & 3.4 & 5.0 & 5.0 & 5.0 \\
\hline Consumer price index (end of period) $2 /$ & 8.6 & 3.5 & 4.0 & 5.0 & 5.0 & 5.0 \\
\hline Import volume growth, goods, and services & 13.6 & 8.7 & 5.5 & 5.8 & 6.6 & 7.2 \\
\hline Import value growth, goods, and services & 9.5 & 2.9 & 12.5 & 9.1 & 12.0 & 7.8 \\
\hline Export volume growth, goods, and services & -1.5 & 5.9 & 11.0 & 10.5 & 9.7 & 10.5 \\
\hline Export value growth, goods, and services & 0.5 & 12.8 & 8.8 & 5.6 & 15.5 & 8.3 \\
\hline Terms of trade, goods, and services (Base year 2000) & 6.5 & 12.1 & -7.3 & -7.4 & 0.2 & -2.7 \\
\hline Ksh per US\$ exchange rate (end of period) $3 /$ & 77.2 & 81.9 & 81.0 & & & \\
\hline \multirow{2}{*}{\multicolumn{7}{|c|}{$\begin{array}{l}\text { Nominal effective exchange rate (- depreciation; end } \\
\text { of period) }\end{array}$}} \\
\hline & -10.4 & -1.2 & $\ldots$ & $\ldots$ & $\ldots$ & $\ldots$ \\
\hline Real effective exchange rate (- depreciation; end of period) & -4.3 & -2.4 & $\cdots$ & $\cdots$ & $\cdots$ & $\cdots$ \\
\hline \multicolumn{7}{|l|}{ Money and credit } \\
\hline $\begin{array}{l}\text { M3 (broad money and foreign currency deposits, } \\
\text { end period) }\end{array}$ & 13.0 & 26.2 & 19.2 & 16.6 & 14.1 & 14.7 \\
\hline \multicolumn{7}{|l|}{ Investment and saving } \\
\hline Investment & 19.9 & 20.5 & 23.1 & 24.5 & 25.2 & 25.2 \\
\hline Central government & 7.5 & 8.9 & 10.3 & 10.6 & 11.1 & 10.9 \\
\hline Other & 12.4 & 11.6 & 12.8 & 13.9 & 14.1 & 14.3 \\
\hline Gross national saving & 11.9 & 15.1 & 15.8 & 17.0 & 18.7 & 20.2 \\
\hline Central government $4 /$ & 2.2 & 1.6 & 2.7 & 3.7 & 5.1 & 5.6 \\
\hline Other & 9.7 & 13.6 & 13.1 & 13.3 & 13.7 & 14.6 \\
\hline \multicolumn{7}{|l|}{ Central government budget $5 /$} \\
\hline Total revenue & 22.4 & 22.7 & 25.2 & 25.3 & 25.3 & 25.6 \\
\hline Total expenditure and net lending & 27.8 & 30.1 & 32.9 & 31.8 & 31.2 & 30.8 \\
\hline Overall balance (commitment basis) excluding grants & -5.4 & -7.4 & -7.7 & -6.6 & -5.9 & -5.2 \\
\hline Overall balance (commitment basis) including grants & -4.5 & -6.5 & -6.5 & -5.3 & -4.6 & -3.9 \\
\hline Primary budget balance & -1.5 & -3.9 & -3.8 & -2.7 & -1.9 & -1.2 \\
\hline Net domestic borrowing & 3.1 & 5.5 & 4.6 & 3.2 & 1.3 & 1.2 \\
\hline \multicolumn{7}{|l|}{ Balance of payments } \\
\hline Exports value, goods, and services & 26.6 & 27.3 & 27.9 & 26.0 & 26.6 & 25.8 \\
\hline Imports value, goods, and services & 42.0 & 39.4 & 41.6 & 40.0 & 39.8 & 38.2 \\
\hline Current external balance, including official transfers & -8.0 & -5.3 & -7.3 & -7.5 & -6.5 & -5.0 \\
\hline Current external balance, excluding official transfers & -7.9 & -5.3 & -7.2 & -7.5 & -6.4 & -5.0 \\
\hline \multicolumn{7}{|l|}{ Gross international reserve coverage } \\
\hline In billions of U.S. dollars (end of period) & 3.2 & 3.8 & 4.2 & 4.6 & 5.9 & 7.4 \\
\hline In months of next year imports (end of period) & 3.1 & 3.3 & 3.3 & 3.3 & 3.8 & 4.4 \\
\hline \multicolumn{7}{|l|}{ Public debt 6 / } \\
\hline Total public debt, net (percent of GDP) & 41.7 & 45.7 & 47.1 & 46.9 & 45.1 & 43.6 \\
\hline Of which: external debt & 23.3 & 23.5 & 22.8 & 22.2 & 21.9 & 21.7 \\
\hline Domestic debt, net of deposits & 18.5 & 22.2 & 24.3 & 24.6 & 23.2 & 21.9 \\
\hline
\end{tabular}

Sources: Kenyan authorities; and IMF staff estimates and projections.

1/ Fiscal year is from July 1 st through June 30 th.

2/ The CPI series was revised in November 2009 based on a new methodology.

3/ Actual as of June, 2010.

4/ Revenue plus program grants minus recurrent expenditure.

5/ Coverage increased in FY 2010/11 to incorporate tuitions and fees to universities and hospitals, and associated expenditure.

6/ Refers to the debt of the central government. 


\section{Statement by Mr. Majoro, Executive Director Kenya \\ January 31, 2011}

My Kenyan authorities are facing critical challenges trying to meet the costs of implementing a new constitution, mitigating the impact of climate change, investing in essential infrastructure and maintaining social spending whilst balancing these priorities against the need for gradual fiscal adjustment to achieve long term debt sustainability. Low level of international reserves and the expected deterioration in the terms of trade further compound the challenges. It is in this regard that my authorities request a three year arrangement under the Extended Credit Facility (ECF) to help them address these daunting challenges. We thank the staff for their constructive dialogue with the authorities and the Board and Management for their continued support.

\section{Recent Economic Developments}

Following various shocks that hit the economy in 2008-09, all signals point to recovery. Growth for 2010 is estimated at just over 5 percent, reflecting favorable weather conditions, resilient domestic demand and strong macroeconomic policy implementation. Ample food supply and vigorous competition in the telecommunications sector have helped keep inflation well below the authorities' target of 5 percent. Monetary policy has been accommodative with low interest rates and ample liquidity. The exchange rate has also stabilized. However, international reserves have remained relatively low as capital inflows are modest.

\section{Medium Term Outlook and Policies}

My authorities forecast a buoyant medium term outlook especially with the adoption of the new constitution which is expected to lay the foundation for better governance and stability and usher in renewed investor confidence. Growth is expected to accelerate to $5.7 \%$ in 2011 , driven by increased private sector investment, stepped up public investment and Public Private Partnerships in infrastructure projects.

\section{Fiscal Policy}

My authorities aim to maintain fiscal sustainability in the medium term with the overall fiscal balance declining to 5 percent and to keep the debt ratio at a sustainable level. The key strategy to achieving this goal is to maintain significant concessional external financing and to shift the composition of domestic debt towards longer term maturities to minimize both costs and risk. Currently, the government has lengthened the government bond term to 25 years. The authorities also intend to access the international capital markets with the issuance of a sovereign bond when global financial conditions permit. This is expected to reduce pressure on interest rates with the pickup in credit by the private sector. The authorities also aim to ensure that its domestic borrowing does not crowd out the private sector given the expected increase in private investment as economic activity picks up. Non- 
concessional loans will be limited to investment projects that demonstrate revenue stream and social return.

The authorities aim to maintain a revenue effort of about 25 percent of GDP with commitment to pursue new revenue and compliance initiatives in order to increase revenue collection. The authorities plan to appoint a Tax Reform Commission (TRC) to simplify the tax code in line with best practices, modernize VAT legislation and increase automation of the Kenya Revenue Authority.

In support of critical infrastructure aimed at reducing the cost of doing business and crowding-in private sector investment, my authorities have increased the budgetary allocations to development expenditure while still aiming to contain growth of total expenditure. Further, they intend to prioritize infrastructure investments and enhance the execution rate for both domestically and foreign financed development projects. This will be achieved through: (i) setting up more frequent monitoring and review of projects; (ii) more active engagement of development partners through regular review meetings; and (iii) more rigorous selection criteria for inclusion of new projects in the budget. Expenditure controls will be enhanced through implementation of new Public Finance Management legislation. A draft PFM bill is expected to be submitted to the newly created Commission for the Implementation of Constitution (CIC). My authorities are also committed to institutionalizing and fully deploying the IFMIS on a single integrated platform.

\section{Monetary Policy}

In the medium term, the monetary policy is targeted to deliver low and stable inflation while at the same time ensuring continued stability in long term interest rates as well as maintaining a competitive exchange rate consistent with Kenya's export oriented private sector led growth strategy. It is expected that the ongoing financial sector reforms will enhance the efficiency of the sector as well as improve the monetary policy transmission mechanism. Consistent with the floating exchange rate regime, the authorities intend to continue to allow the exchange rate to be market-determined, with interventions limited to smoothing out excessive exchange rate volatility, and gradually building international reserves to the statutory level of 4 months of import cover. Further, the authorities are committed to reviewing the monetary policy framework in an effort to enhance efficiency of monetary operations.

\section{Financial Stability}

The financial sector has seen increased competition and innovation which has resulted in the expansion of access to financial services for the Kenyan people. The sector has also seen growing cross-border banking services. To reduce attendant risks, my authorities are committed to strengthening the supervision and regulatory framework including introducing legislation to allow for the demutualization of the Nairobi Stock Exchange, completing the regulatory and supervisory framework of Savings and Credit Cooperatives, giving more powers to the Deposit Insurance Fund, strengthening risk-based supervision, expanding supervisory procedures. In addition, my authorities will modify the Banking Act to allow the 
Central Bank to disclose surveillance information of foreign exchange bureaus, enforce compliance of Retirement Benefit Authority rules on National Social Security Fund and fully implement the Crime and Anti-Money Laundering Act of 2009. My authorities will also upgrade the crisis management framework to deal with challenges of cross-border banking activities and continue promoting financial access to the unbanked.

\section{External Sector}

My authorities project deterioration in the terms of trade with the continuing increase in oil prices and decline in Kenya's export prices for tea and coffee. This, together with increased demand for imports of goods and services arising from higher growth, is expected to widen the current account deficit to about 7.5 percent in the next two years. As a result, the buildup of international reserves buffers will be constrained. The authorities are committed to implementing the Common Market Protocol for EAC in order to take full advantage of arising opportunities. Remittances are recovering somewhat, but prospects depend on economic developments in North America and Europe, which account for over 70 percent of total remittance flow to Kenya..

\section{The New Constitution}

The new Kenyan constitution was promulgated on $27^{\text {th }}$ August 2010. The authorities' are in the process of implementing the structure of governance as provided for under the new constitution. Implementation is on track with three Commissions so far established. This includes the Judicial Service Commission (JSC); the Constitution Implementation Commission (CIC) which is mandated to oversee the development of legislation and administrative procedures necessary for the implementation of the new fundamental law; and the Commission on Allocation of Resources (CAR) whose mandate is to have stable and predictable allocations of revenue, promote affirmative action in respect of disadvantaged areas and groups, and develop fiscal capacity and efficiency for county governments. More new structures will be created in the course of the Fund program and the associated costs will be known as they are gradually established. The authorities are confident that the implementation of new constitution and reforms under agenda IV of National Accord will be sufficient to diffuse social and political tensions in the run-up to elections in 2012.

\section{Conclusion}

After being buffeted by the post-election conflict, drought and the global economic crisis, the Kenyan economy is now gradually recovering. However significant challenges still remain, related to the expected weakening of the external position and the need for resources to implement the new constitution and build essential infrastructure. My authorities' goal is to rebuild their international reserves and achieve debt sustainability, while expanding needed infrastructure and paying for the implementation of the new constitution. The proposed ECF is carefully designed to assist them to achieve their development objectives. 